\title{
NONLINEAR OPTICS AND DYNAMICS IN SEMICONDUCTOR LASERS
}

T. B. Simpson

J. M. Liu

Jaycor Corporation

P.O. Box 85154

San Diego CA 92186-5154

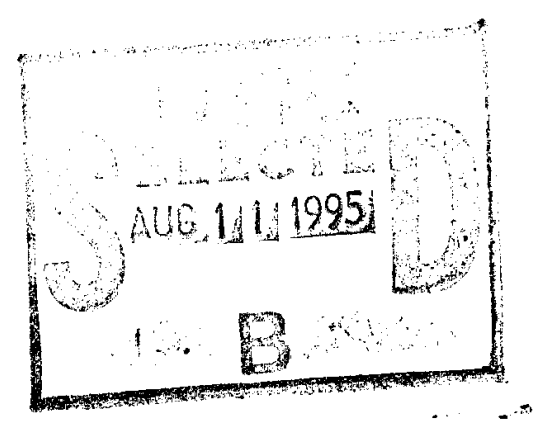

September 1994

Final Report

APPROVED FOR PUBLIC RELEASE; DISTRIBUTION IS UNLIMITED.

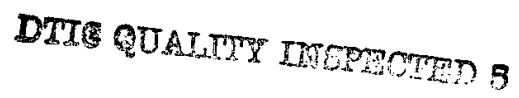

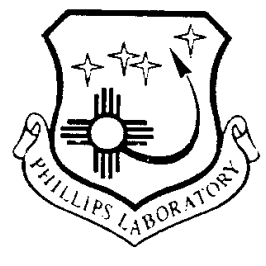

PHILLIPS LABORATORY

Lasers and Imaging Directorate

AIR FORCE MATERIEL COMMAND

KIRTLAND AIR FORCE BASE; NM 87117-5776 
This final report was prepared by the Jaycor Corp, P. O. Box 85154, San Diego, CA 92186 under Contract F29601-90-C-0068, Job Order 33260706 with the Phillips Laboratory, Kirtland Air Force Base, New Mexico. The Laboratory Project Officer-in-Charge was Christopher M.
Clayton (LIDN).

When Government drawings, specifications, or other data are used for any purpose other than in connection with a definitely Government-related procurement, the United States Government incurs no responsibility or any obligation whatsoever. The fact that the Government may have formulated or in any way supplied the said drawings, specifications, or other data, is not to be regarded by implication, or otherwise in any manner construed, as licensing the holder, or any other person or corporation; or as conveying any rights or permission to manufacture, use, or sell any patented invention that may in any way be related thereto.

This report has been authored by a contractor of the United es Government. Accordingly, the United States Government retains a nonexclusive, royalty-free license to publish or reproduce the material contained herein, or allow others to do so, for the United States Government purposes.

This report has been reviewed by the Public Affairs Office and is releasable to the National Technical Information Service (NTIS). At NTIS, it will be available to the general public, including
foreign nationals.

If your address has changed, if you wish to be removed from the mailing list, or if your organization no longer employs the addressee, please notify PL/LIDN, 3550 Aberdeen Ave SE, Kirtland AFB, NM 87117-5776, to help maintain a current mailing list.

This report has been reviewed and is approved for publication.

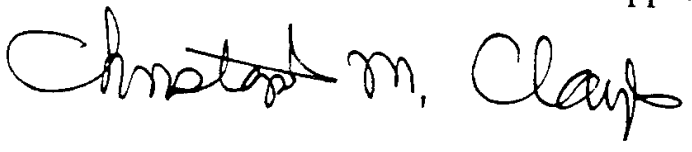

\section{CHRISTOPHER M. CLAYTON} Project Officer

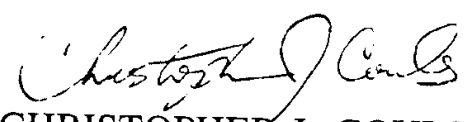

CHRISTÓPHER J. COULS

Lt Col, USAF

Chief, Laser Systems Division
FOR THE COMMANDER

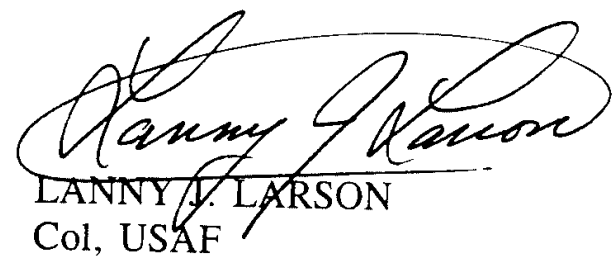

Director, Lasers and Imaging Directorate 


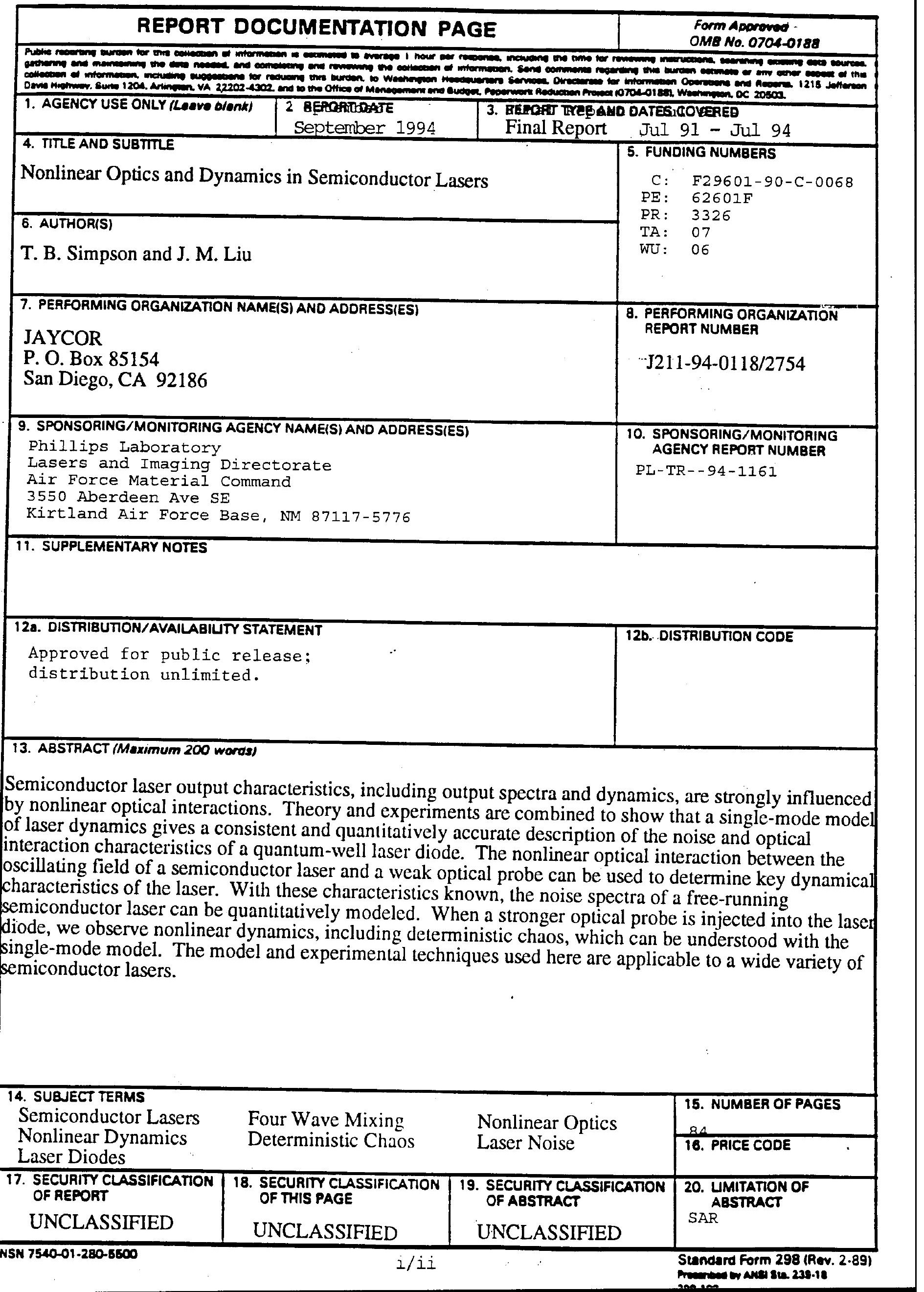




\section{ACKNOWLEDGEMENTS}

The authors have benefited from many useful discussions and an ongoing collaboration with Dr. Chris Clayton, Dr. Tom Gavrielides, and Dr. Vassilios Kovanis of the Nonlinear Optics Center of the Air Force's Phillips Laboratory. In particular, much of the work in the stronginjection regime was done in close collaboration with the Phillips Laboratory scientists.

Dr. Kovanis did the numerical calculations discussed in Sections 7.2 and 7.3 and prepared several of the figures used there. In addition, Mr. Frank Doft provided expert assistance in the experimental work. The vertical-cavity surface-emitting lasers were provided by Dr. Kai Feng Huang of National Chiao Tung University, Hsinchu, Taiwan.

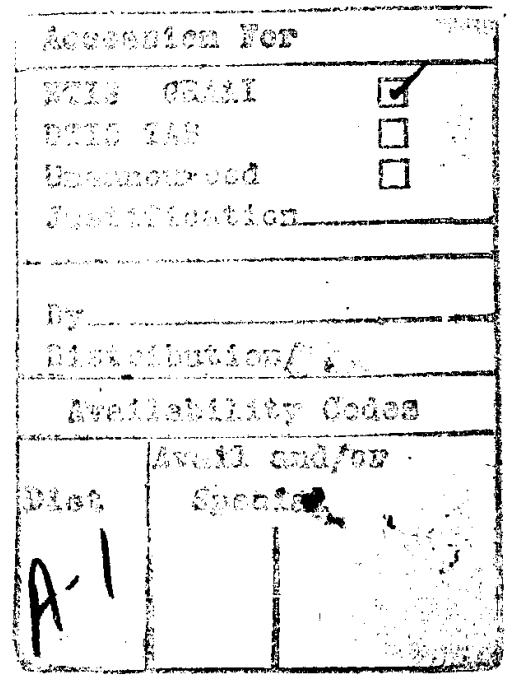




\section{CONTENTS}

Section

Page

SUMMARY

INTRODUCTION

DYNAMICAL MODEL OF A SEMICONDUCTOR LASER

EXPERIMENTAL APPARATUS

5.0 FOUR-WAVE MIXING AND OPTICAL MODULATION IN A SEMICONDUCTOR LASER

5.1 INTRODUCTION

5.2 THEORETICAL ANALYSIS ……………………........................... 13

5.3 EXPERIMENTAL VERIFICATION ..................................................

5.4 LASER PARAMETER CHARACTERIZATION ................................ 25

5.4.1 Principle and Experimental Approach

5.4.2 Procedure and Accuracy of Parameter D................................. 25

5.4 .3 Power Dependecuracy of Parameter Determination ............... 26

\subsection{DISCUSSION}

NOISE IN A SEMICONDUCTOR LASER

7.1 INTRODUCTION

7.2 PERIOD-DOUBLING ROUTE TO CHAOS

7.3 PERIOD-DOUBLING CASCADES AND CHAOS

7.4 DISCUSSION

DETUNED INJECTION IN THE STRONG SIGNAL REGIME

56

9.0

10.0

VERTICAL-CAVITY SURFACE-EMITTING LASERS

CONCLUSIONS

REFERENCES 


\section{FIGURES}

\section{Figure}

The optical power spectrum of the interaction laser diode when operated in a free-running condition at an injection current level of $40 \mathrm{~mA}$.

Low resolution spectra of the side modes of the laser diode at three different operating currents

Schematic diagram of the experimental setup

Phasor diagram illustrating the relationship between the contributing components and the spectral symmetry or asymmetry of the signals in the four-wave mixing and optical modulation process

Spectral characteristics of the regenerative and four-wave mixing fields and the amplitude and phase modulations as a function of the detuning

Importance of phase modulations and the parameter, $b$

Typical four-wave mixing spectra from the output of the injected laser

Experimentally measured and theoretically calculated regeneratively amplified and four-wave mixing signals from the optical spectral analysis as a function of the detuning frequency

Experimentally measured and theoretically calculated power signal as a function of the detuning frequency

Representative spectrum of the regenerative reflectivity at a laser output

Representative power spectrum at a laser output power of $9.2 \mathrm{~mW}$

Sensitivity of the regenerative reflectivity spectrum to the value of $\gamma_{r}$

Sensitivity of the regenerative reflectivity spectrum to the value of $b$

Power dependence of the square of the resonance frequency.

Power dependencies of $\gamma_{r}$ and $\gamma_{p}$

Log-log plot of the measured values of features in the optical spectra

as a function of the normalized injection, $\xi$.

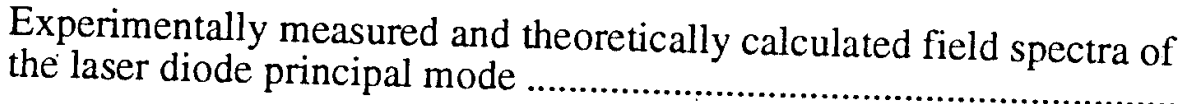

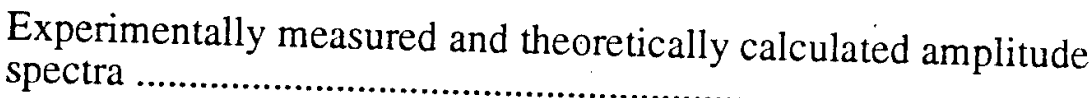




\section{FIGURES (Continued)}

Figure

Page

19 Experimentally measured side mode field spectrum and Lorentzian curve fit with a half-width half-maximum linewidth of $220 \mathrm{MHz}$.

20 Optical spectra of a semiconductor laser under optical injection at two levels in a period-doubling route to chaos

21 Numerically obtained bifurcation diagram of the extrema of the normalized optical field amplitude, $a(t)$, versus the normalized injection level, $\xi$, showing the period-doubling route to chaos

Variation of the resonance frequency as a function of the injection level $\xi$

23 Numerically calculated bifurcation diagram of the extrema of the normalized optical field amplitude, $a(t)$, versus the normalized injection level, $\xi$

24 Measured power optical spectra of the quantum well laser under optical injection at six levels of injection power

A mapping of the regions of different laser dynamics as a function of injection level and detuning

Calculated changes in the amplitude noise spectra of a single-mode laser, where the dominant noise source is spontaneous emission, due to stable injection locking by an external laser whose frequency is tuned so that $\phi=0$.

Measured changes in the amplitude noise spectra in the vicinity of the free-running relaxation-resonance peak, due to stable injection locking by an external laser whose frequency is tuned so that the amplitude of the noise spectra is minimized

The frequency spacing between the two orthogonally polarized modes as a function of injection current.

Relative output powers of the two polarization components, and singlepolarization operation, as a function of injection current

The linewidths of the lowest order spatial modes as a function of injection current

31 The low frequency microwave spectra of the output of the VCSEL when detected by a fast photodiode 


\section{FIGURES (Continued)}

Figure

32

The frequency of the resonance due to polarization switching as a function of the injection current.

Page

33 The dependence of the resonance peaks observed in the microwave spectra, regardless of polarization mode of operation, as a function of the injection current 


\subsection{SUMMARY}

Semiconductor lasers have many important technological applications and are often used as a test structure for models of the operation of laser oscillators. External optical signals can change the output characteristics of the semiconductor laser through the nonlinear interaction of optical fields within the laser cavity. This work attempts to present a consistent picture of the nonlinear optical interactions within a free-running laser diode and one subject to near-resonant external optical injection. We concentrate on measurements and modeling of a nearly single-mode, quantum-well laser diode but our techniques can be applied to a wide variety of laser structures.

The phase and amplitude characteristics of nearly degenerate four-wave mixing in a semiconductor laser are studied. This type of interaction occurs when the injected signal is sufficiently weak that a linearized dynamical model can be used to describe the optical interaction. There is a direct connection between nearly degenerate four-wave mixing in a semiconductor laser and optical small-signal modulation in the laser field. It can be understood using a model of an unlocked, optically injected laser which emphasizes the effect of the laser resonator on the optical interactions. This model correctly describes the observed spectral characteristics and their dependence on the intrinsic parameters of the semiconductor laser. This is used to develop a simple and accurate technique using a single experimental setup for the parasitic-free characterization of the intrinsic laser parameters, including the relaxation resonance frequency, the total relaxation rate, the nonlinear relaxation rate, and the linewidth enhancement factor. Other parameters, such as the spontaneous carrier lifetime, the photon lifetime, the differential and nonlinear gain parameters, and the $K$ factor, are determined from the power dependencies of these parameters. This technique requires only two continuous-wave (cw) lasers closely matched in wavelength and is applicable to semiconductor lasers of any wavelength and any dynamic bandwidth.

Exploiting similarities in the nonlinear optical interactions underlying the noise and external optical modulation spectra in laser diodes, we quantitatively model the noise spectra of a nearly single-mode laser diode. Two types of nonlinear optical mixing are prominent. The first involves the nonlinear interaction between the spontaneous and coherent fields in the oscillating mode. The second is the scattering of the coherent field by the carrier fluctuations induced by the amplified spontaneous emission in the weak side modes.

Experimental measurements and a single-mode analysis of the quantum-well laser diode when it is subject to resonant, strong optical injection are combined to demonstrate that the diode follows 
a period-doubling route to chaos. All laser parameters used in the model, including the influence of spontaneous emission noise, were experimentally determined based on the four-wave mixing technique. The transition to chaos can be used to reduce the uncertainty in the value of one of the model parameters, the linewidth enhancement factor. The laser diode exhibits chaotic dynamics over a bounded range of injection levels. As the chaotic regime is approached from both lower and higher injection levels, it follows the period-doubling route to chaos, though the route is largely obscured by spontaneous-emission noise. A new, bounded regime of perioddoubling occurs for injection levels well above the region of chaotic dynamics. Further, at injection levels above the chaotic region, optical injection strongly modifies the carrier-field resonance coupling frequency. The single-mode model, including spontaneous emission noise, displays the dominant characteristics of the optical spectra.

The nonlinear dynamics induced by external optical injection are a strong function of the frequency difference between the injecting and interaction lasers as well as the optical injection level. There is a complicated mapping of the dynamics as a function of frequency offset and injection level. At all injection levels, however, there is a range of injection frequencies where the interaction laser can be frequency-locked (with good stability characteristics) to the injection laser. In this region of stable injection locking, the laser displays improved modulation characteristics relative to its free-running conditions.

Finally, we present preliminary measurements of the noise characteristics of a vertical-cavity surface-emitting laser, including novel spectra induced by rapid, spontaneous polarization switching of the optical output. These results point to the fact that many new phenomena can be expected when the semiconductor laser operates with more than one strong output mode. 


\subsection{INTRODUCTION}

Nonlinear optical interactions in a variety of material systems have been investigated for applications in optical communications, optical signal processing, and coherent propagation of high-intensity laser beams. In many applications, the ideal nonlinear optical material has a fast response time and a low threshold for nonlinear optical interaction. This combination has proven to be elusive in bulk materials. Low threshold materials, such as the photorefractive crystals, have a slow response time. To achieve the high optical intensities required in many nonlinear materials, the use of waveguides to achieve high intensities over long optical interaction lengths has been proposed. This has led to a wide range of material systems being used in waveguide configurations.

One of the most interesting results was achieved in one of the most technologically important waveguide systems, the semiconductor laser or laser diode. Nakajima and Frey were the first to report the observation of the generation and amplification of signals through nonlinear optical interactions in a Fabry-Perot laser diode [Refs. 1,2]. They observed generation of conjugate signals through four-wave mixing in the laser diode. These signals were more than a factor of $10^{3}$ stronger than the input signal. Early theoretical efforts attempted to explain the nonlinear interaction that induced these signals by concentrating on the spatial nature of the interaction [Refs. 3,4]. Agrawal was the first to describe the importance of the modulation of the carrier density, but his model only qualitatively explained the data [Ref: 5]. Another early model concentrated on the temporal dynamics of the interaction rather than the spatial interaction. This model achieved better, though still only qualitative, agreement with the data [Ref. 6].

In a previous report, we described our initial observations of the phase and amplitude characteristics of the signals generated by the nonlinear optical interaction [Ref. 7]. Before that time, the experimental data consisted primarily of data on the magnitude of the optical spectra of the generated signals. We noted that, under some circumstances the nonlinear optical interaction led to an almost pure phase modulation of the output of the semiconductor laser under investigation. This result could not be explained by the spatial interaction model.

At the same time, we realized that there was a striking similarity between the four-wave mixing spectra and the noise spectra of a free-running laser diode [Ref. 8]. Semiconductor lasers have been extensively studied as a test system for understanding the fundamental noise properties of laser oscillators [Ref. 9]. The noise spectra of laser diodes have been generally modeled by concentrating on the temporal rather than the spatial nature of the interaction. Due to the 
coupling between the free carriers and the optical field in a laser diode, the noise spectra do not exhibit a simple Lorentzian line shape [Ref. 8]. Modeling of diode operation has successfully explained the qualitative features of the noise spectra [Refs. 10, 11].

This report describes our studies of the nonlinear optical interaction in laser diodes and the importance of the nonlinear optical coupling to both the generation of four-wave mixing signals and the generation of the noise spectrum of a free-running laser. We also show how the nonlinear optical coupling can lead to full, nonlinear dynamics, including deterministic chaos, under appropriate conditions. The report is divided into several parts.

In the next section, we describe our dynamical model of the operation of a semiconductor laser under the influence of external optical sources and noise sources. It is a model which concentrates on the temporal nonlinear coupling of the optical field and the free carriers, the gain medium, of the semiconductor laser. We then describe the experimental apparatus used to test the model. Our apparatus appears to be unique in that we are able to make measurements which separately quantify the phase and amplitude characteristics of the generated signals. We do this by simultaneously monitoring both the optical spectra and the power spectra of the signals that arise from the nonlinear optical interaction. Under appropriate conditions, the nonlinear dynamical equations can be linearized to describe the effect of a weak external optical signal on the output of a laser diode, Section 5.0. Through this interaction, key dynamical parameters of laser operation can be determined. In fact, all of the parameters necessary for a comparison of the full nonlinear model with an operational laser can be determined in this weak injection limit. We next use these parameters to similarly model the noise spectrum of the laser diode. Section 7.0 describes the dynamics that result under strong, resonant optical injection. A bounded region of chaotic dynamics is observed. Section 8.0 describes key findings of the nonlinear interaction and dynamics under strong, near-resonant optical injection. Section 9.0 describes some preliminary measurements made on a novel laser, the vertical-cavity surfaceemitting laser (VCSEL). These are initial findings on work that is still in progress. Finally, we draw some conclusions and suggestions for future work. 


\subsection{DYNAMICAL MODEL OF A SEMICONDUCTOR LASER}

In a variety of laser systems, a set of coupled equations for the complex oscillating field and the inversion density describes the nonlinear dynamics. An isolated, single-mode laser of this type, such as a semiconductor laser, is sufficiently described by only two rate equations, one for the photon density and another for the carrier density [Refs. 12,13]. When the laser is coupled to the outside world and subject to external sources, such as spontaneous emission noise or external optical signals, an equation for the complex, oscillating electric field must replace the simpler equation for the photon density. Over the past three years, we have developed a model for semiconductor laser operation which describes the dynamics of a nearly single-mode laser diode. This model draws strongly on past work describing the noise spectra of semiconductor lasers, injection-locking in semiconductor lasers, and the effects of weak external optical feedback. It is based on coupled equations for the complex field and the carrier density,

$$
\begin{aligned}
& \frac{d A}{d t}=-\frac{\gamma_{c}}{2} A+i\left(\omega_{0}-\omega_{c}\right) A+\frac{\Gamma}{2}(1-i b) g A+\eta A_{i} e^{-i \Omega t}+F_{s p}, \\
& \frac{d N}{d t}=\frac{J}{e d}-\frac{N}{\tau_{s}}-\frac{2 \varepsilon_{0} n^{2}}{\hbar \omega_{0}} g|A|^{2},
\end{aligned}
$$

where $A$ is the total complex intracavity field amplitude at the free-oscillating frequency $\omega_{0}, \omega_{C}$ is the longitudinal-mode frequency of the cold laser cavity, $\Gamma$ is the confinement factor, $b$ is the linewidth enhancement factor, $g$ is the gain coefficient, $A_{i}$ is the complex amplitude, $\Omega$ is the detuning of the injection signal, $F_{S p}$ is the spontaneous emission Langevin noise source and is assumed to have a correlation time short compared to $\gamma_{c}^{-1}$ [Ref. 10], $N$ is the carrier density, $J$ is the injection current density, $e$ is the electronic charge, $d$ is the active layer thickness, $\tau_{s}$ is the carrier lifetime, and $n$ is the refractive index of the semiconductor medium.

For Equations 1 and 2 to apply, it is assumed that the semiconductor laser can be modeled as a single-mode system. All spatial effects are contained in the confinement factor, $\Gamma$, and all interacting optical fields are assumed to be confined to the same spatial mode. The gain, $g$, and the linewidth enhancement factor, $b$, relate the complex field and the polarization induced by the free carriers which make up the gain medium [Ref. 5],

$$
P=-\varepsilon_{0} \frac{n^{2}}{\omega_{0}}(b+i) g A e^{-i \omega_{0} t}
$$


This relation between $P$ and the complex field assumes that the polarization adiabatically follows the field [Ref. 12].

The exact functional dependence of the gain on parameters such as the carrier density and optical fields is unknown, but it is important to consider the gain coefficient $g(N, S)$ as a function of the carrier density and the intracavity photon density, $S$. Therefore, we make the assumption that the gain can be modeled perturbatively about an operating point determined by the injection current, $J$, carrier density, $N_{O}$, and field amplitude, $A_{O}$, when there are no external optical sources or noise sources present, and

$$
S \simeq \frac{2 n^{2} \varepsilon_{0}}{\hbar \omega_{0}}\left|A_{0}\right|^{2} .
$$

In this model, the characteristics of the spectra generated by the nonlinear optical interaction can be directly connected to many fundamental parameters of the laser diode, such as the relaxation resonance frequency, the total relaxation rate, the nonlinear relaxation rate, and the linewidth enhancement factor. The total relaxation rate, $\gamma_{r}$, of the laser has contributions from the carrier relaxation and the differential and nonlinear gain parameters [Refs. 13, 14]. It can be written as

$$
\gamma_{r}=\gamma_{s}+\gamma_{n}+\gamma_{p}
$$

where $\gamma_{s}=1 / \tau_{s}$ is the spontaneous carrier relaxation rate and $\gamma_{n}$, and $\gamma_{p}$ are relaxation rates contributed by the differential and nonlinear gain parameters, respectively. While $\gamma_{s}$ is a constant at a given carrier density, $\gamma_{n}$ and $\gamma_{p}$ are proportional to the intracavity photon density through the following relations:

$$
\gamma_{n}=g_{n} S=\left.\frac{\partial g}{\partial N}\right|_{N_{0}} S \text { and } \gamma_{p}=-\Gamma g_{p} S=-\left.\Gamma \frac{\partial g}{\partial S}\right|_{N_{O}} S
$$

where $g_{n}$ and $g_{p}$ are the differential and nonlinear gain parameters defined as the derivatives of the gain coefficient, $g$, with respect to the carrier density and the photon density, respectively, evaluated at the operating point. The relaxation resonance frequency, $\Omega_{r}$, depends on these parameters through the following relation:

$$
\Omega_{r}=2 \pi f_{r}=\left(\gamma_{c} \gamma_{n}+\gamma_{s} \gamma_{p}\right)^{1 / 2}
$$




\begin{abstract}
All modeling in the following sections is derived from this basic model and these definitions. Additional assumptions required to apply to the different limiting conditions that we have experimentally explored are detailed in the different sections. Here, we conclude with two points. First, the external optical signal and the spontaneous-emission noise source are treated in a very similar manner by the model. Second, because the optical field is complex, there are really two equations, one for each of the two quadrature components, or equivalently, for the amplitude and the phase, embedded in Equation 1. Therefore, the semiconductor laser subject to optical injection contains the three dynamical degrees of freedom that are necessary but not sufficient for the observation of deterministic chaotic dynamics [Ref. 12].
\end{abstract}




\subsection{EXPERIMENTAL APPARATUS}

There is voluminous literature describing measurements of spectral characteristics of semiconductor lasers. To relate the observed spectra to models of semiconductor laser dynamics, measurements concentrated on determining what parameters needed to be included in the model to reproduce the observed spectral features. Early measurements focused on the Lorentzian line shape of central peak in the output spectrum. From these measurements, the importance of the linewidth enhancement factor, $b$, was deduced [Ref. 9]. Measurements of the intensity spectrum showed that the laser gain had to be a function of both the carrier density and the circulating intensity [Ref. 15].

Most of the measurements have been performed to extract the qualitative nature of the spectra and were not accurate enough to make a precise quantitative comparison with theoretical models. Recently, there has been considerable effort directed towards a more precise determination of the key model parameters from the measured spectra [Refs. 13,14,16]. All of these studies concentrated on either the optical or the amplitude spectra of the laser diodes and, therefore, were not able to determine key parameters without additional estimates of the semiconductor laser properties. One of the key features of our work is the quantitative comparison of theory and data without this need for additional assumptions. Therefore, details of the experimental apparatus and technique that we used are important.

The laser diodes used for the bulk of this work were two Spectra Diode Labs (San Jose, CA) SDL-5301-G1 single transverse and nearly single longitudinal mode GaAs/AlGaAs quantumwell lasers. These laser diodes have a cavity length of $500 \mu \mathrm{m}$ and coated end facets with nominal reflectivities of $\geq 0.95$ for the rear surface and $\leq 0.04$ for the output coupler. Both lasers were temperature and current stabilized. To bring them to near-degenerate operating frequencies, the temperature was adjusted for gross frequency changes and then the current was adjusted for fine tuning of the frequency offset $f$. Overlap of frequencies could be achieved over a limited range of operating conditions for the two lasers. The laser used as the interaction laser could be operated at currents from 32 to $50 \mathrm{~mA}$, corresponding to output powers from 4 to $15 \mathrm{~mW}$. The threshold current was $23.3 \mathrm{~mA}$ and a linear light versus injection current level was measured. The pump parameter, $\tilde{J}$, is defined as the difference between the operating current and the threshold current, normalized to the threshold current. It followed the relation $\tilde{J}=$ $0.078 P$. Here, $P$ is the output power in milliwatts. The lasers were overlapped at wavelengths of 827.5 to $827.7 \mathrm{~nm}$. The wavelength was measured using a wave meter. 
A typical optical spectrum of the principal mode, measured using techniques described next, is shown in Figure 1 along with a calculated Lorentzian line shape curve. The central linewidth is calculated to be $10 \mathrm{MHz}$, full width at half maximum. The exact value of the linewidth is unimportant for the spectral shape at offset frequencies greater than $\sim 100 \mathrm{MHz}$. There is significant deviation of the optical spectrum from the Lorentzian line shape in the wings. These are the relaxation resonance features [Ref. 8]. Though the laser diode operates on one principal mode, with $\sim 90$ percent of the total output power, there are many weak side modes. Figure 2 shows spectra taken with a $0.5 \mathrm{~m}$ Spex scanning monochrometer. No single side mode is dominant and the spectrum shifts with injection current.

Figure 3 shows the experimental setup for our key measurements. In most measurements, the output of one laser diode (LD1), the master or injection laser at a frequency $v_{l}$, was passed through an acoustooptic modulator where a fraction, on the order of 10 percent, of the beam was deflected and shifted in frequency by $80 \mathrm{MHz}$. The shifted beam at $v_{l}+80 \mathrm{MHz}$ was used to probe a second laser (LD2), the injected or slave laser, oscillating at $v_{0}=\omega_{0} / 2 \pi$. Thus the frequency offset $f=\Omega / 2 \pi$ is given by $v_{O}+f=v_{l}+80 \mathrm{MHz}$ and can be changed by varying $v_{l}$. By frequency shifting the injection beam in this way, the unshifted beam from the master laser

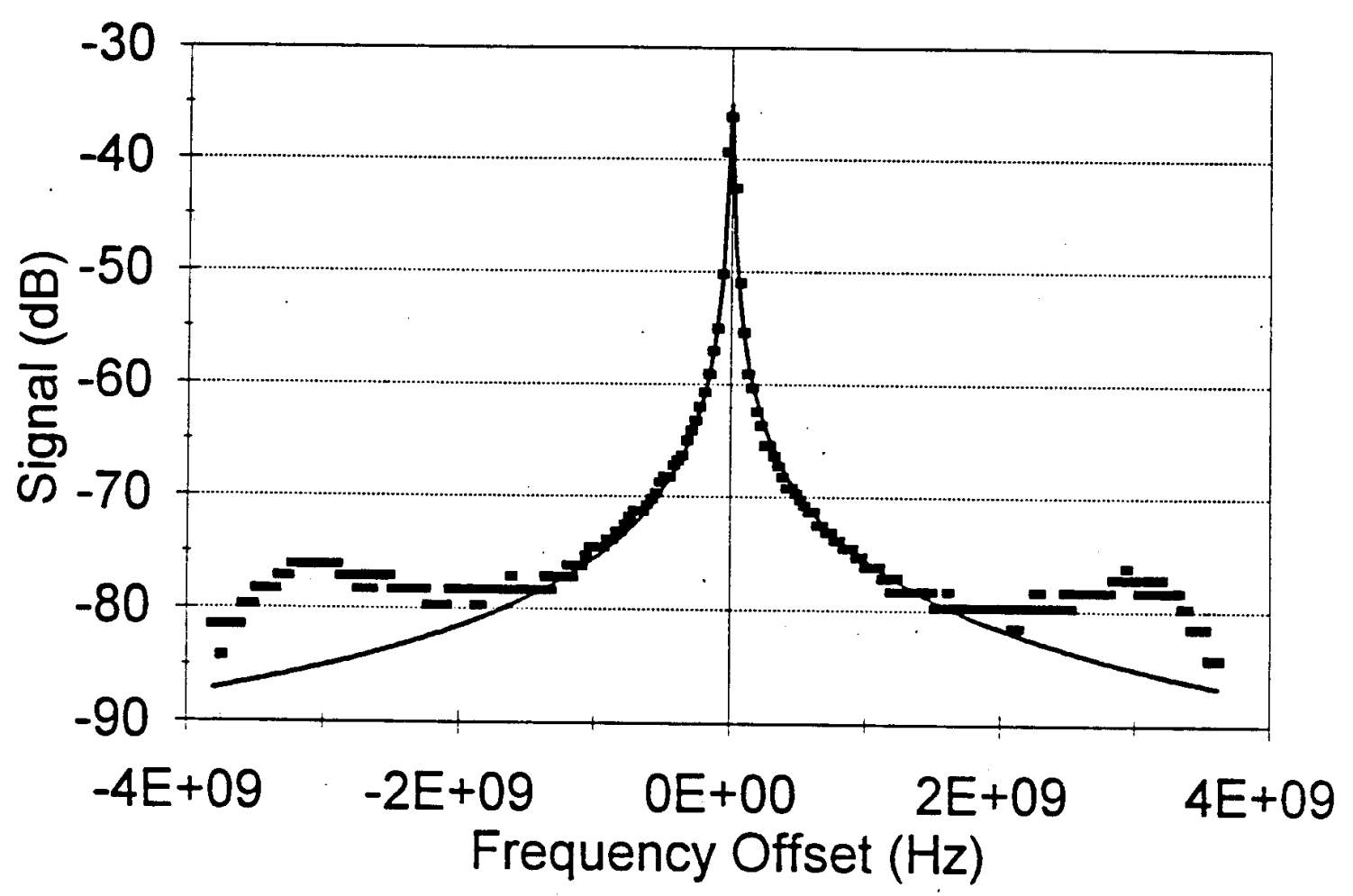

Figure 1. The optical power spectrum of the interaction laser diode when operated in a free-running condition at an injection current level of $40 \mathrm{~mA}$. Also shown is a calculated Lorentzian line shape using a 10-MHz linewidth. 


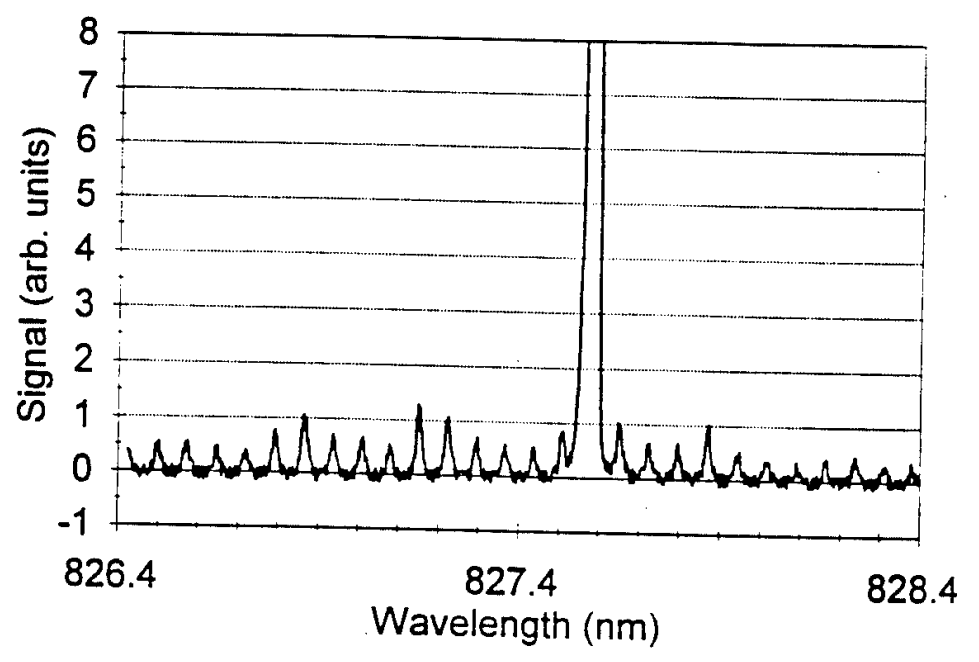

(a) Current $=32 \mathrm{~mA}$

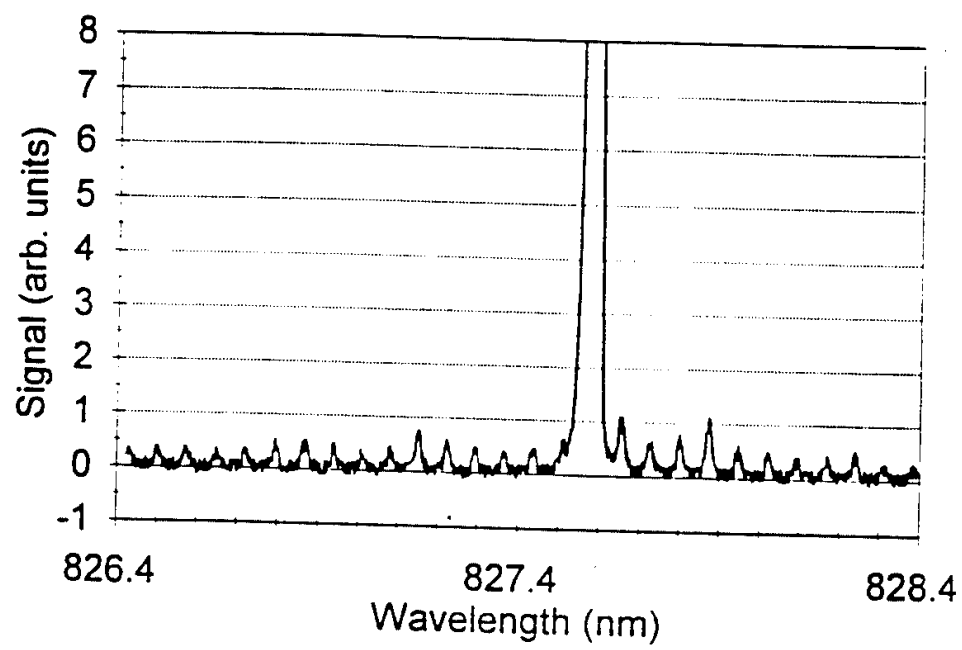

(b) Current $=40 \mathrm{~mA}$

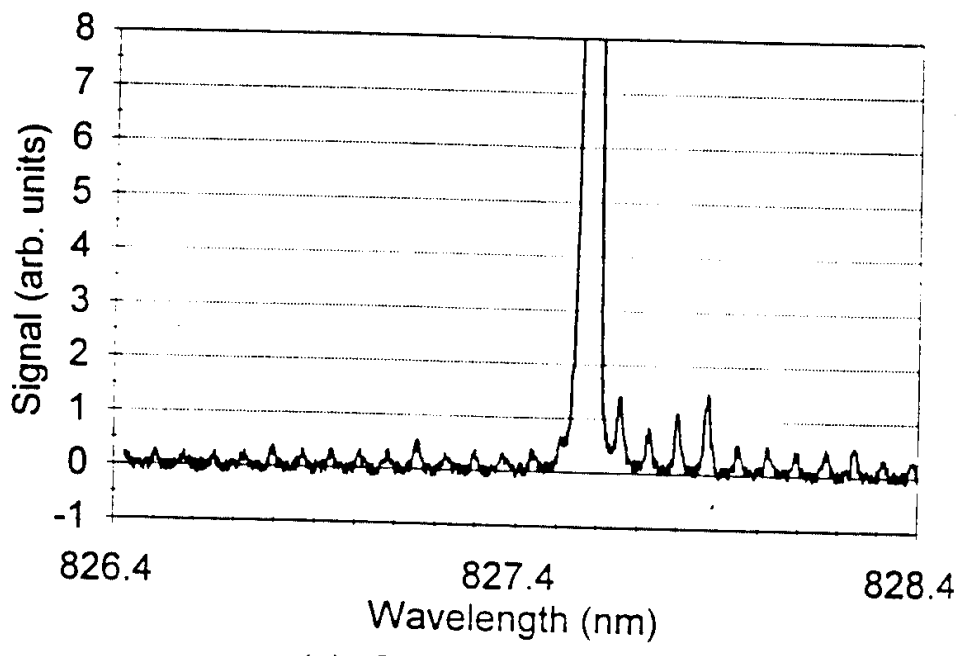

(c) Current $=48 \mathrm{~mA}$

Figure 2. Low resolution spectra of the side modes of the laser diode at three different operating currents. The principal mode has a peak value of 95,190 , and 265 , respectively, for (a), (b), and (c). 


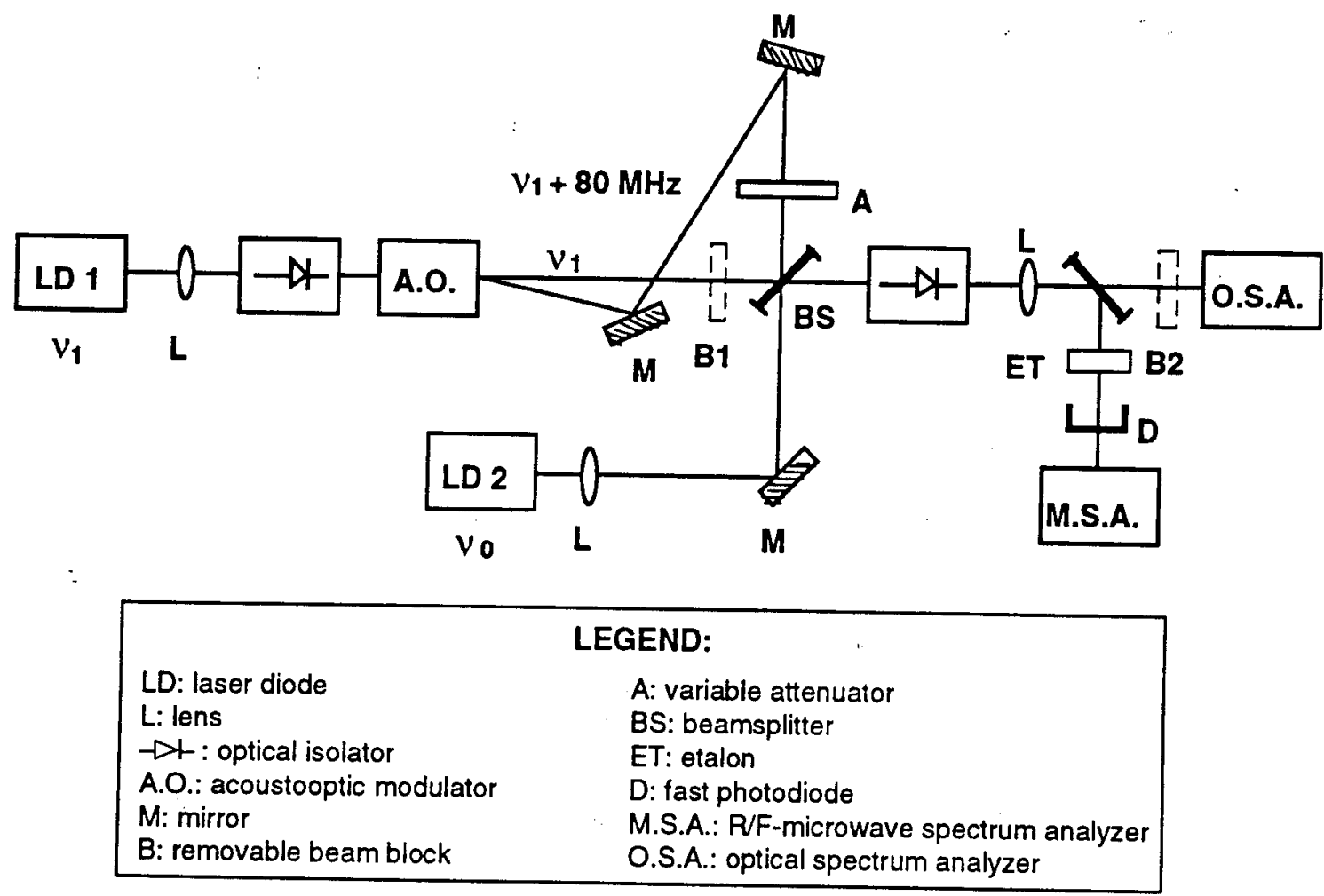

Figure 3. Schematic diagram of the experimental setup.

could be used as a local oscillator for heterodyne measurements. Optical isolators, with $\sim 60-\mathrm{dB}$ isolation, were used to avoid mutual injection and to reject back-reflected light from any components in the optical path. When very high injection levels were required, the acoustooptic modulator was removed and the steering mirror was moved into the main path of the master laser. In this configuration, the injection laser could not be directly monitored and could not be used as a local oscillator for heterodyne detection. Removal of the acoustooptic modulator did not lead to noticeable feedback effects on the master oscillator.

The output of both lasers could be monitored using both optical and microwave/radio-frequency spectrum analyzers to measure both the optical field and amplitude spectra. Measuring both spectra simultaneously allowed us to distinguish between amplitude and phase modulations of the output of the injected laser. The amplitude spectrum was obtained by detecting the output of the injected laser with a fast photodiode of $7 \mathrm{GHz}$ frequency response and displaying the photodiode signal on the spectrum analyzer. To separate the amplitude signal of the principal oscillating mode from the weak side modes, an etalon with a free spectral range of $\sim 400 \mathrm{GHz}$ and a finesse of -20 was placed in front of the fast photodiode. The optical spectrum could be measured in two ways. The direct measurement used a Newport SR-240C scanning Fabry-Perot which has a free spectral range of $2 \mathrm{THz}$ and a finesse of greater than 50,000, giving an optical 
frequency resolution of about $40 \mathrm{MHz}$. Alternatively, the unshifted beam from the master oscillator could be mixed with the signal beam from the slave laser. The microwave spectrum analyzer was then set at a fixed offset frequency. Setting the offset frequency to $80 \mathrm{MHz}$, and sweeping the injection frequency by varying the bias current to the master laser, we measured a heterodyne spectra of the optical signal from the slave laser generated at the injection frequency. Alternatively, the injecting beam could be blocked and the microwave spectrum analyzer could be set to some convenient frequency, typically $\sim 5 \mathrm{GHz}$, and the heterodyne measurement yielded an optical spectrum of the free-running slave laser. This latter measurement technique is a variant of the measurement technique used in Reference 13.

Depending upon the specific experiment, beam blocks were inserted or removed to allow either, or both, of the two lasers to pass to the detection apparatus and allow the master laser to inject light into the slave laser. Data from the optical spectrum analyzer were recorded using a Tektronix 7854 digitizing oscilloscope while the microwave spectrum analyzer, a Tektronix 495, included digitization. Data files were downloaded onto a personal computer for further analysis.

While the experimental configuration shown in Figure 3 has allowed us to make a variety of precise measurements on semiconductor lasers, it does have limitations. For our work, the most significant was the difficulty achieving frequency overlap between the master and slave lasers. Temperature and current allow considerable control of the lasers but they do not provide continuous control of the frequency. This problem can be overcome by using one of the tunable, external cavity semiconductor lasers that have recently become commercially available. We also found that some care had to be exercised with the current bias conditions of the two lasers. Specifically, the master laser had to be operated much farther above threshold than the slave laser. This was required to keep the noise spectrum of the master laser from influencing the measurements of the slave laser. This problem can also be overcome by using one of the external cavity semiconductor lasers. These lasers have much reduced noise spectra relative to conventional, edge-emitting Fabry-Perot lasers. 


\subsection{FOUR-WAVE MIXING AND OPTICAL MODULATION IN A SEMICONDUCTOR LASER}

\subsection{INTRODUCTION}

Many interesting phenomena, such as injection locking, four-wave mixing, and chaotic behavior, can be observed when a semiconductor laser is injected with an external optical signal under different conditions. When the injection signal is weak and is outside of the injection-locking frequency range, a four-wave mixing signal can be observed while the injection signal is regeneratively amplified [Refs. 1, 17]. In a traveling-wave semiconductor laser amplifier, a four-wave mixing signal as strong as the output of the amplified injection signal can be generated [Ref. 17]. In a Fabry-Perot laser cavity, these signals are enhanced by the resonant effect of the laser cavity in addition to the amplifying effect of the laser gain medium. Earlier attempts to include the effects of the cavity in the theoretical description [Refs. 3,6] failed to correctly reproduce the spectral characteristics of the signals. In particular, the asymmetry in the spectrum of the regeneratively amplified signal and the differences between that and the spectrum of the four-wave mixing signal were not recognized. These analyses also failed to make correct connections between the experimentally observed spectral characteristics and various parameters of the laser oscillator.

In an unlocked, driven GaAs microwave oscillator, parametric sidebands can be generated and the output spectra also appear to be asymmetric. This has been reported and theoretically explained using an unlocked oscillator model by Stover [Ref. 18]. Recently, we showed that the spectral characteristics of nearly degenerate four-wave mixing in a semiconductor laser can be correctly described using a model of an unlocked, optically injected laser which takes into account the phase and amplitude constraints imposed by the laser resonator on the optical fields [Ref. 19]. In this section, we show that the same process can be understood in terms of the amplitude and phase modulations in the laser field induced by the injection signal. We make connection between the optical modulation and the four-wave mixing signals and study in detail the symmetry and asymmetry of their spectra. From the dependence of the characteristics of these spectra on the intrinsic laser parameters, the four-wave mixing experiment can be used for parasitic-free characterization of many important laser parameters, such as the relaxation resonance frequency, the relaxation rate, the differential and nonlinear gain parameters, the linewidth enhancemient factor, and the carrier and photon lifetimes. We also show here the simplicity and accuracy of this characterization technique. Throughout this section, we consider . the nondegenerate injection of a weak signal at an angular frequency $\omega_{0}+\Omega$ into a single-mode 
semiconductor laser oscillating at $\omega_{0}=2 \pi v_{0}$ with a frequency offset $\Omega=2 \pi f$ outside of the injection-locking range. This is the limit where Equations 1 and 2 can be linearized and the noise source term can be ignored.

\subsection{THEORETICAL ANALYSIS}

The output field of the injected laser consists of spectral components at the free-oscillating frequency $\omega_{0}$, the injection frequency at $\omega_{0}+\Omega$, and the four-wave mixing parametric frequency $\omega_{0}-\Omega$. From the viewpoint of regenerative amplification and four-wave mixing, it can be mathematically described by

$$
E(t)=A(t) e^{-i \omega_{0} t}+c . c .
$$

with

$$
A(t)=A_{0}+A_{r} e^{-i \Omega t}+A_{f} e^{i \Omega t}
$$

where $A_{O}$ is the steady-state field amplitude at the oscillating frequency $\omega_{0}$, and $A_{r}$ and $A_{f}$ are the complex amplitudes of the regeneratively amplified and four-wave mixing fields, respectively. Alternatively, it can be described by

$$
E(t)=E_{0}\left[1+\sigma \cos \left(\Omega t-\varphi_{1}\right)\right] \cos \left[\omega_{0} t+\delta \cos \left(\Omega t-\varphi_{2}\right)\right]
$$

as a field undergoing both amplitude and phase modulations, where

$$
\hat{\sigma}=\sigma e^{i \varphi_{1}} \text { and } \hat{\delta}=\delta e^{i \varphi_{2}}
$$

are the complex amplitudes of the amplitude and phase modulations, respectively. The two pictures are connected through

$$
. \hat{\sigma}=\frac{A_{r} A_{O}^{*}+A_{f}^{*} A_{O}}{\left|A_{O}\right|^{2}} \text { and } \hat{\delta}=i \frac{A_{r} A_{O}^{*}-A_{f}^{*} A_{O}}{\left|A_{O}\right|^{2}}
$$


or

$$
\frac{A_{r}}{A_{0}}=\frac{\hat{\sigma}-i \hat{\delta}}{2} \text { and } \frac{A_{f}^{*}}{A_{0}^{*}}=\frac{\hat{\sigma}+i \hat{\delta}}{2}
$$

Thus, both $A_{r}$ and $A_{f}$ have contributions from both the amplitude and phase modulations of the laser field.

The physical origin of the four-wave mixing, or optical modulation, process is the carrier pulsation in the semiconductor gain medium caused by the beating between the mixing optical fields. In a single-mode semiconductor laser, the spatial gratings are not important and the process is solely contributed by the temporal pulsation of the carrier density at the beat frequency [Refs. 5, 19]. To the first order, the carrier density can be expressed as

$$
N(t)=N_{O}+N_{l} e^{-i \Omega t}+N_{l}^{*} e^{i \Omega t}
$$

where $N_{O}$ is the steady-state carrier density without perturbation and $N_{l}$ is the amplitude of the carrier pulsation.

To reveal the physical mechanisms involved in the process, we first use Equations 2 and 14 to obtain

$$
N_{l}=-\frac{\gamma_{c}-\gamma_{p}}{\gamma_{r}-\gamma_{p}-i \Omega} \frac{S}{\Gamma} \hat{\sigma}
$$

This indicates that the carrier pulsation is caused by the amplitude fluctuation of the total optical field in the laser cavity. When $\Omega \rightarrow 0, N_{I}$ and $\hat{\sigma}$ are out of phase by $\pi$. This means that the induced carrier pulsation acts to reduce the amplitude fluctuation in the laser field caused by the optical injection. As a result, the amplitude modulation decreases drastically as $\Omega \rightarrow 0$. In the small detuning range very close to degeneracy where $|\Omega|<\Omega_{r}$, the four-wave mixing spectra are contributed primarily by the phase modulation.

Using Equations 9 and 15 to solve Equation 1, we have

$$
\frac{A_{r}}{A_{O}}=\frac{i}{\Omega}\left(\frac{1-i b}{2} \frac{\Omega_{r}^{2}-i \Omega \gamma_{p}}{\gamma_{c}-\gamma_{p}} \frac{\Gamma N_{1}}{S}+\frac{\eta A_{i}}{A_{O}}\right)
$$




$$
\frac{A_{f}^{*}}{A_{0}^{*}}=\frac{i}{\Omega}\left(\frac{1+i b}{2} \frac{\Omega_{r}^{2}-i \Omega \gamma_{p}}{\gamma_{c}-\gamma_{p}} \frac{\Gamma N_{I}}{S}\right)
$$

From Equation 12, we further obtain

$$
\begin{aligned}
& \hat{\sigma}=\frac{i}{\Omega}\left(\frac{\Omega_{r}^{2}-i \Omega \gamma_{p}}{\gamma_{c}-\gamma_{p}} \frac{\Gamma N_{1}}{S}+\frac{\eta A_{i}}{A_{0}}\right), \\
& \hat{\delta}=\frac{i}{\Omega}\left(b \frac{\Omega_{r}^{2}-i \Omega \gamma_{p}}{\gamma_{c}-\gamma_{p}} \frac{\Gamma N_{I}}{S}+i \frac{\eta A_{i}}{A_{0}}\right) .
\end{aligned}
$$

These relations show the various physical components of contribution to the regeneratively amplified and four-wave mixing signals, as well as those to the amplitude and phase modulations of the field. The factor

$$
G(\Omega)=\frac{\Omega_{r}^{2}-i \Omega \gamma_{p}}{\gamma_{c}-\gamma_{p}} \frac{\Gamma N_{l}}{S}
$$

appears in all four relations and is the gain caused by the carrier pulsation. This can be easily seen from the fact that when the nonlinear gain coefficient is negligible so that $\gamma_{p}=0$, $G=g_{n} \Gamma N_{l}$. In a semiconductor medium, a change of gain by $G$ is always accompanied by a change of refractive index by $b G$. Thus, an amplification of the amplitude modulation by $G$ is accompanied by an amplification of the phase modulation by $b G$. This is seen in Equations 18 and 19. Both amplitude and phase modulations also have contributions from the externally injected field. In contrast, the externally injected field directly contributes only to the regeneratively amplified signal at the same frequency, but not to the four-wave mixing signal at a different frequency. It is only through the carrier pulsation that the external field induces the four-wave mixing signal. This is seen by using Equations 15 and 18 to obtain

$$
N_{l}=\frac{\gamma_{c}-\gamma_{p}}{\Omega^{2}-\Omega_{r}^{2}+i \Omega \gamma_{r}} \frac{S}{\Gamma} \frac{\eta A_{i}}{A_{O}}
$$

which can be inserted into Equation 17. The modulation on the laser field at $\omega_{0}$ by the gain fluctuation $G$ and the accompanying refractive-index fluctuation $b G$ at $\Omega$ generates sidebands at 
$\omega_{0}+\Omega$ and $\omega_{0}-\Omega$ with equal efficiency although different phases. Thus, the regeneratively amplified signal and the four-wave mixing signal both have contributions from $G$ and $b G$ with equal magnitude but different phases.

The spectral symmetry characteristics of the magnitudes and phases of $A_{r}, A_{f}, \hat{\sigma}$, and $\hat{\delta}$ depend on the phase relationship between the contributing components. This can be illustrated using a phasor diagram shown in Figure 4. For the convenience of illustration, we have defined

$$
\begin{aligned}
& R(\Omega)=-i \Omega \frac{A_{r}(\Omega)}{A_{0}}=\frac{1-i b}{2} G(\Omega)+I, \\
& F^{*}(\Omega)=-i \Omega \frac{A_{f}^{*}(\Omega)}{A_{0}}=\frac{1+i b}{2} G(\Omega), \\
& \Sigma(\Omega)=-i \Omega \hat{\sigma}=G(\Omega)+I \\
& \Delta(\Omega)=-i \Omega \hat{\delta}=b G(\Omega)+i I
\end{aligned}
$$

where $I=\eta A_{i} / A_{0}$. Figure 4 shows the various phasors at $\Omega=\Omega_{r}$ and $-\Omega_{r}$, respectively. The phase angle between $G\left(\Omega_{r}\right)$ and $I$ has the same magnitude but opposite signs as that between $G\left(-\Omega_{r}\right)$ and $I$. Therefore, $\hat{\sigma}$ is symmetric in magnitude but antisymmetric in phase with respect to $\Omega=0$, while $\hat{\delta}$ is asymmetric both in magnitude and phase. For $b \neq 0$, both the magnitude and phase of $A_{r}$ become asymmetric while the magnitude of $A_{f}^{*}$, but not the phase, remains symmetric. Because $b>0$, we expect

$$
\left|A_{r}\left(-\Omega_{r}\right)\right|>\left|A_{f}\left(-\Omega_{r}\right)\right|=\left|A_{f}\left(\Omega_{r}\right)\right|>\left|A_{r}\left(\Omega_{r}\right)\right|
$$

which will be verified with the experimental data in the next section.

Using Equation 21, we can obtain the following spectral characteristics from Equations 16 to 19:

$$
\frac{A_{r}}{A_{o}}=\frac{i}{\Omega} \frac{\Omega^{2}-\Omega_{r}^{2} / 2-b \Omega \gamma_{p} / 2+i\left(\Omega \gamma_{r}-\Omega \gamma_{p} / 2-b \Omega_{r}^{2} / 2\right)}{\Omega^{2}-\Omega_{r}^{2}+i \Omega \gamma_{r}} \frac{\eta A_{i}}{A_{0}}
$$




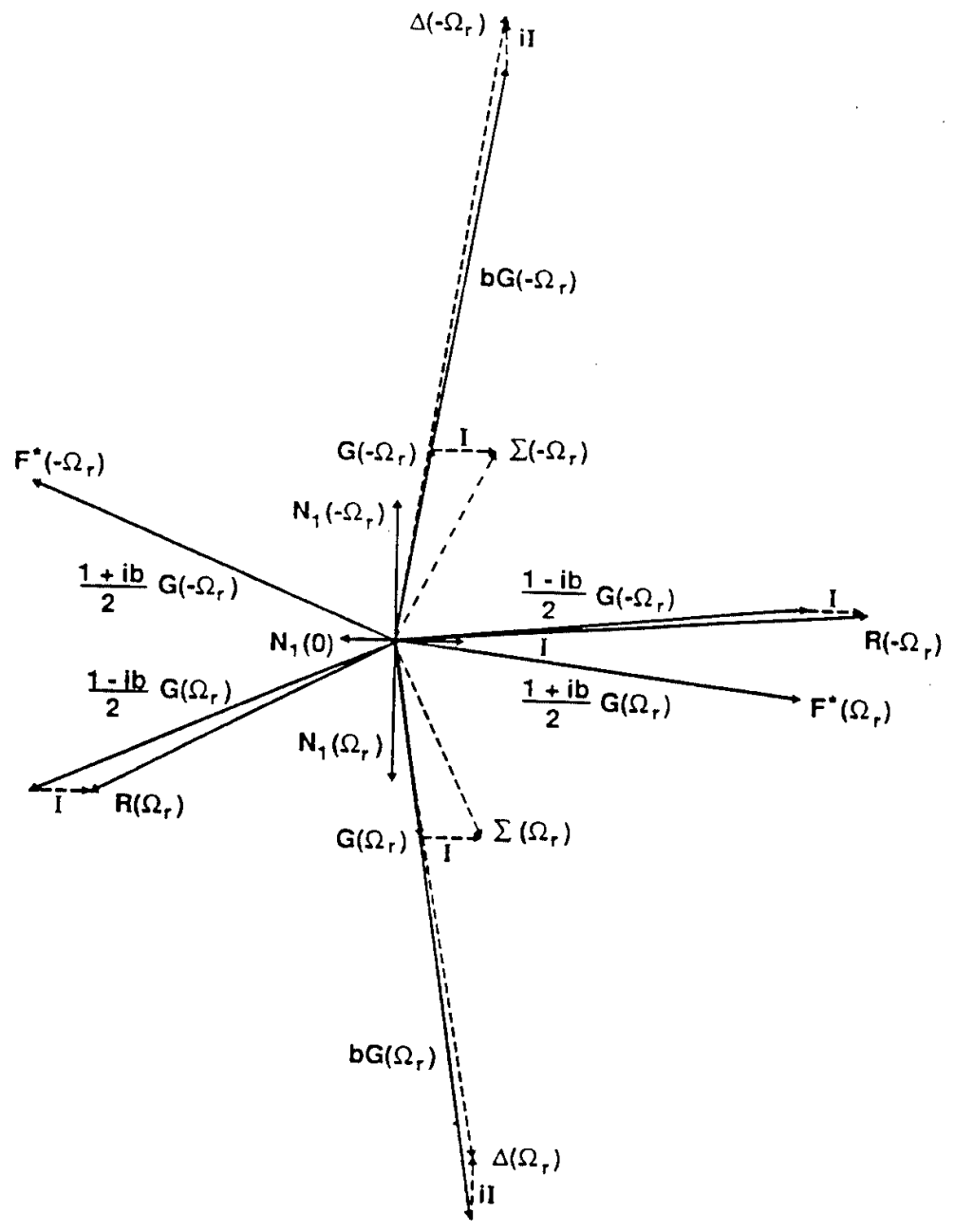

Figure 4. Phasor diagram illustrating the relationship between the contributing components and the spectral symmetry or asymmetry of the signals in the four-wave mixing and optical modulation process.

$$
\frac{A_{f}^{*}}{A_{0}^{*}}=\frac{i}{\Omega} \frac{\left(\Omega_{r}^{2}+b \Omega \gamma_{p}\right) / 2+i\left(b \Omega_{r}^{2}-\Omega \gamma_{p}\right) / 2}{\Omega^{2}-\Omega_{r}^{2}+i \Omega \gamma_{r}} \frac{\eta A_{i}}{A_{0}}
$$

and

$$
\begin{aligned}
& \hat{\sigma}=i \frac{\Omega+i\left(\gamma_{r}-\gamma_{p}\right)}{\Omega^{2}-\Omega_{r}^{2}+i \Omega \gamma_{r}} \frac{\eta A_{i}}{A_{O}}, \\
& \hat{\delta}=-\frac{1}{\Omega} \frac{\Omega^{2}-\Omega_{r}^{2}-b \Omega \gamma_{p}+i\left(\Omega \gamma_{r}-b \Omega_{r}^{2}\right)}{\Omega^{2}-\Omega_{r}^{2}+i \Omega \gamma_{r}} \frac{\eta A_{i}}{A_{0}} .
\end{aligned}
$$


The magnitudes and phases of these spectral characteristics are plotted in Figure 5(a) and (b), respectively, using the following laser parameters as an example: $f_{r}=3 \mathrm{GHz}, \gamma_{r}=5.5 \times 10^{9} \mathrm{~s}^{-1}$, $\gamma_{p}=2.6 \times 10^{9} \mathrm{~s}^{-1}$, and $b=3$. These figures demonstrate the spectral symmetry or asymmetry of the magnitudes and phases of $A_{r}, A_{f}, \hat{\sigma}$, and $\hat{\delta}$ discussed previously. We should emphasize again that the model assumes optical injection outside of the locking range. The discontinuities at $\Omega=0$ are always outside of the range of applicability of this model. However, the injection locking range depends on the intensity of the injection field and can be made arbitrarily small with a very weak injection field.

Figure 6(a) shows the relative contributions of amplitude and phase modulations in the process as a function of the detuning frequency. For a detuning frequency less than the relaxation resonance frequency, the phase modulation significantly dominates the amplitude modulation, as was expected in the earlier discussions. Near zero frequency detuning, the output of the injected laser becomes almost purely phase modulated. The relative importance of phase and amplitude modulations near the relaxation resonance frequency depends strongly on the value of $b$. This is because the carrier pulsation contributes to the amplitude modulation directly through a change of gain $G$ but contributes to the phase modulation indirectly through an accompanying change of refractive index $b G$. Thus the amplitude modulation is independent of $b$, but the phase modulation is a function of $b$. Figure $6(\mathrm{~b})$ shows the phase modulation spectrum at various values of $b$. In case $b=0$, the phase modulation is caused only by the beating of the oscillating laser field with the injection field while the carrier pulsation still contributes to the amplitude modulation. As a result, its spectrum becomes symmetric with respect to $\Omega=0$ and loses the relaxation resonant peaks. For large values of $b$, the contribution of the carrier pulsation to the phase modulation strongly dominates that of the beating between the injection and oscillating fields. Thus the phase modulation spectrum also becomes less asymmetric as the value of $b$ increases.

\subsection{EXPERIMENTAL VERIFICATION}

Nearly degenerate four-wave mixing experiments have been carried out in the weak injection limit where the injection signal acts only as a perturbation to the injected free-running laser. Both the optical and power spectra of the output from the injected laser were measured. The optical spectrum records $\left|A_{r}\right|^{2}$ and $\left|A_{f}\right|^{2}$, each of which has contributions from both phase and amplitude modulations. The power spectrum records $\sigma^{2}$ and thus measures only the amplitude modulation [Ref. 19]. 


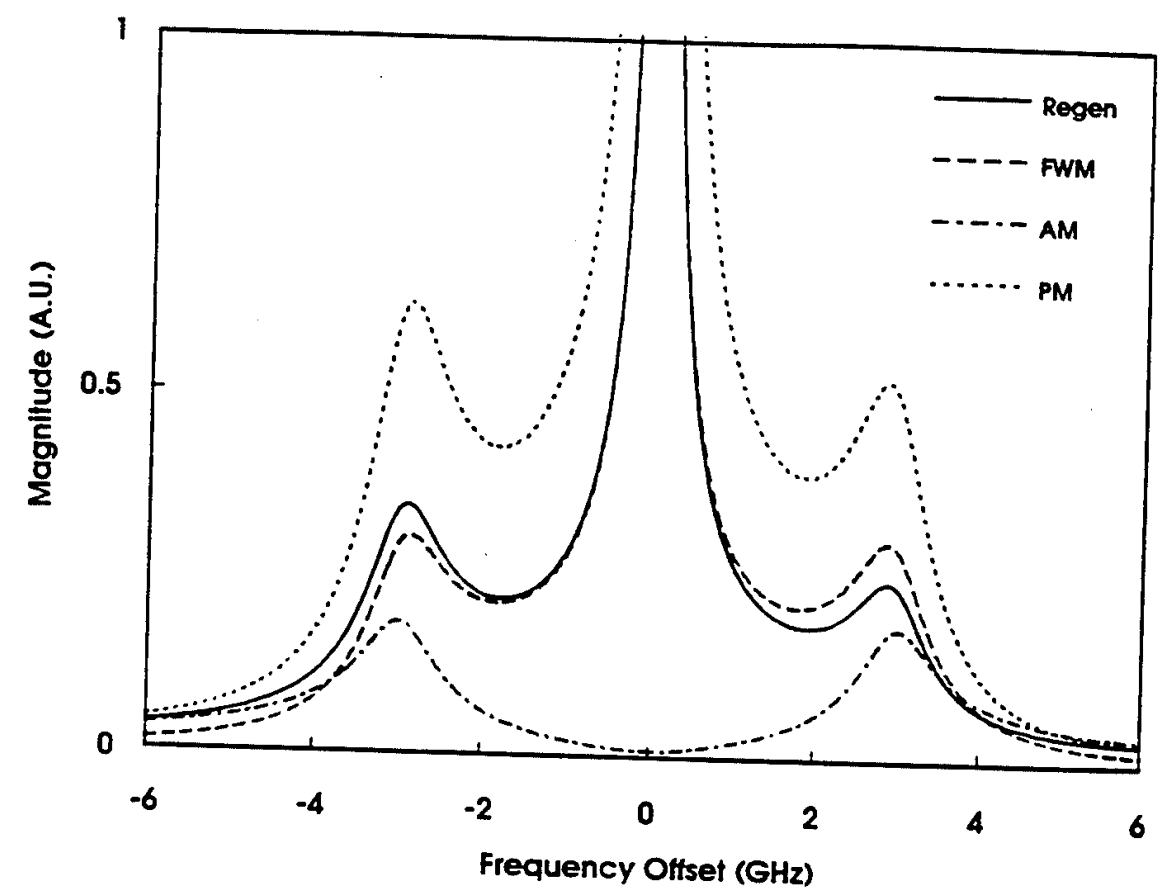

(a) Amplitude

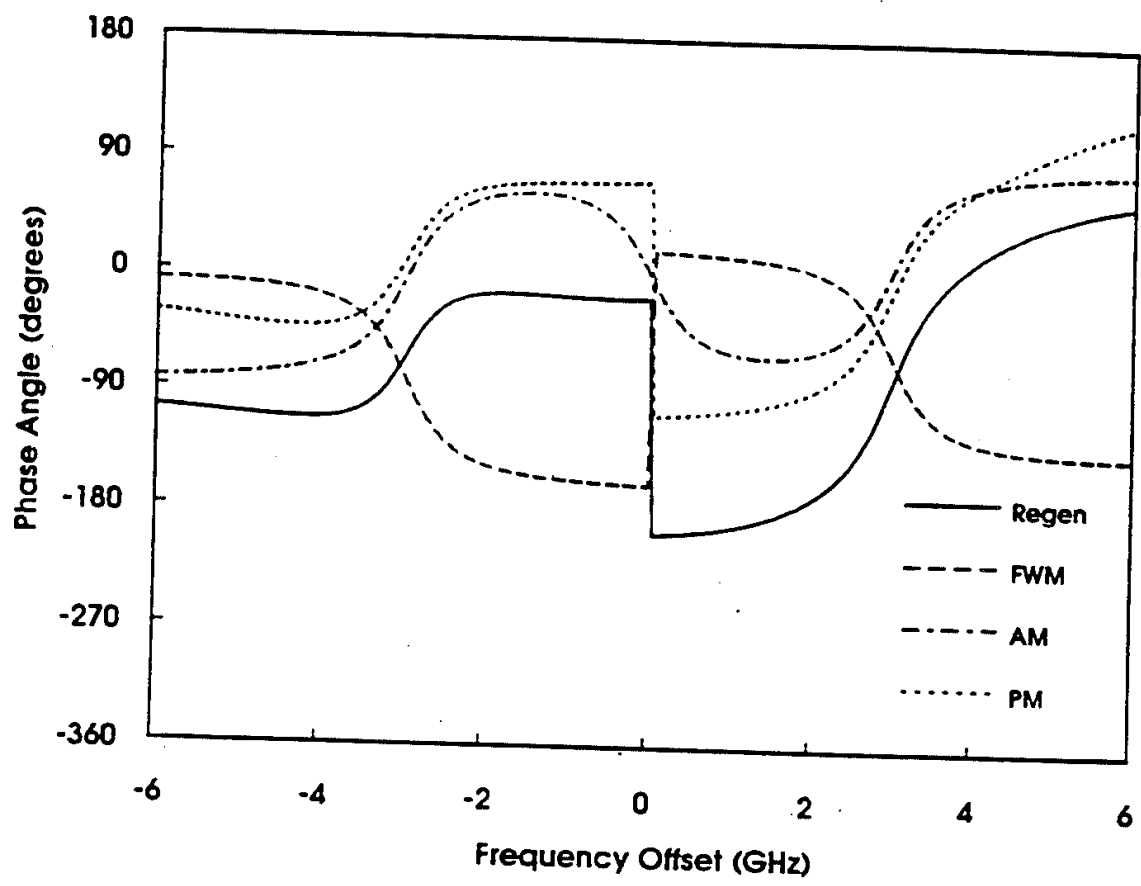

(b) Phase

Figure 5. Spectral characteristics of the regenerative and four-wave mixing fields and the amplitude and phase modulations as a function of the detuning frequency. The laser parameters used are $f_{r}=3 \mathrm{GHz}, \gamma_{r}=5.5 \times 10^{9} \mathrm{~s}^{-1}, \gamma_{p}=2.6 \times 10^{9} \mathrm{~s}^{-1}$, and $\mathbf{b}=3$. This model is valid for optical injection outside of the injection locking range which depends on the injection level. The data are not valid at $\Omega=0$,
which is always within the locking range. 


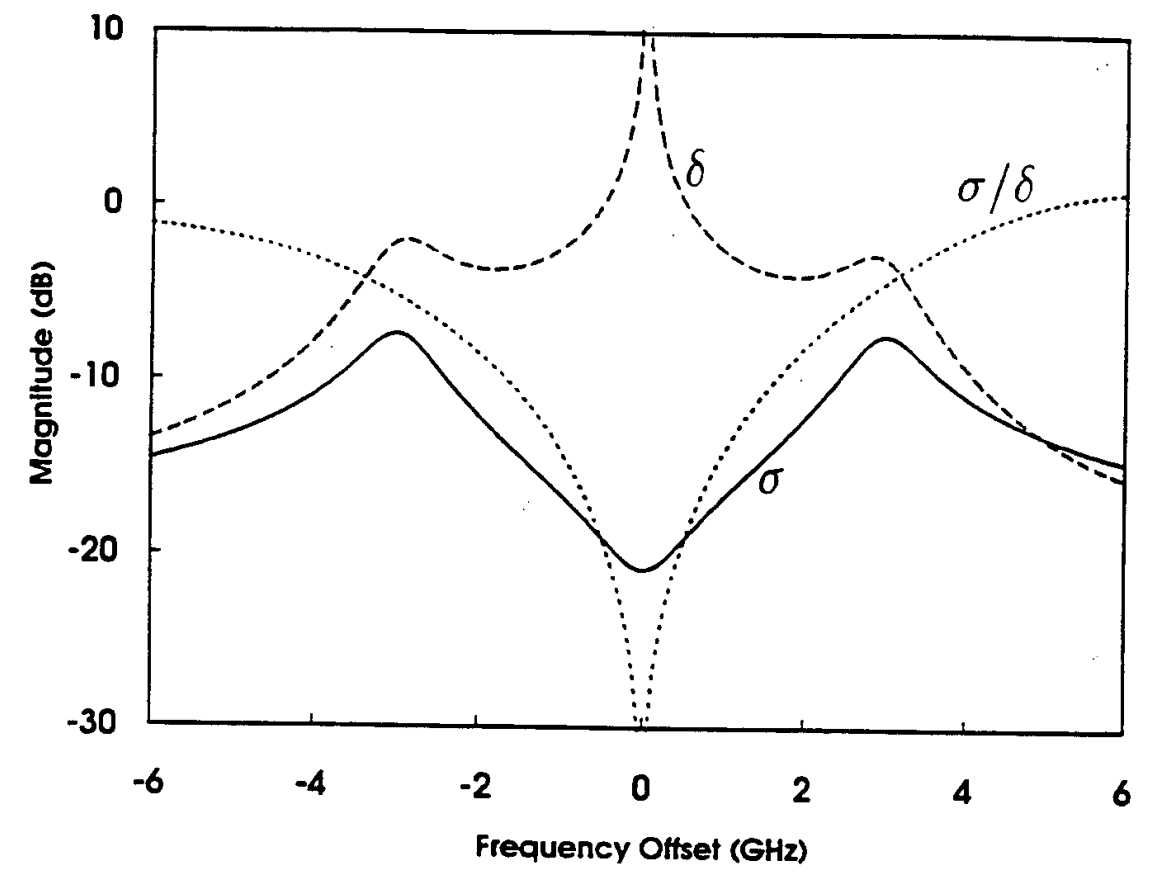

(a) Amplitude, $\sigma$, phase, $\delta$, and ratio spectra

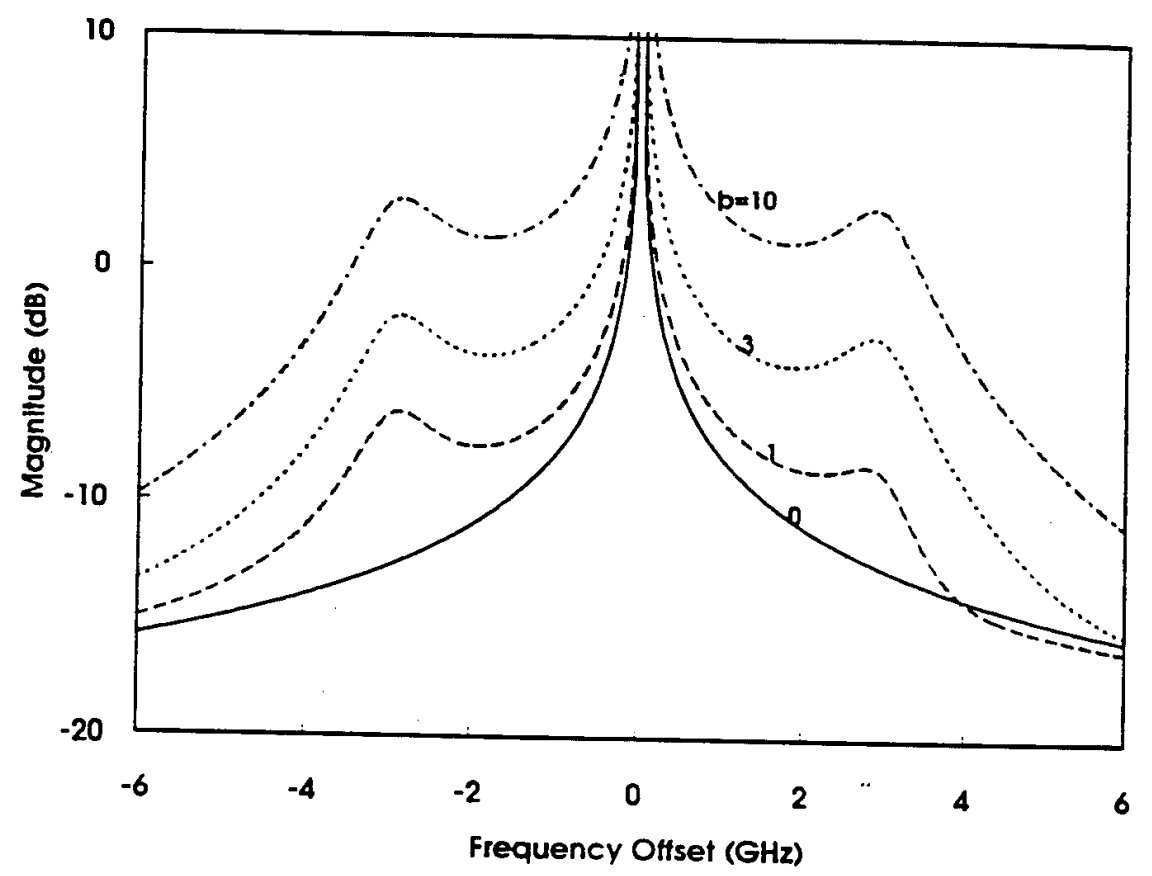

(b) Phase modulation spectra

Figure 6. Importance of phase modulations and the parameter, $b$. (a) Comparison of the amplitude and phase modulations as a function of the detuning frequency for $b=3$. (b) Phase modulation spectrum at various values of $b$. The other laser parameters and the validity of the model are the same as those in Figure 5. 
We first show in Figure 7 two typical optical spectra of the output from the injected laser. These spectra were taken with the injection signal having an approximately constant intensity but with the detuning frequency, $f$, having opposite signs. In both spectra, the regeneratively amplified injection signal and the four-wave mixing signal are equally and oppositely offset from the oscillating frequency, $v_{0}$, of the injected laser. Comparing the two spectra, it can be seen that the four-wave mixing signal is symmetric as a function of detuning on both sides of the oscillating frequency, but the regeneratively amplified injection signal exhibits a strong asymmetry, being stronger on the lower frequency side with $f<0$. As discussed earlier and indicated in Equation 26, the four-wave mixing signal is actually stronger than the regenerative signal when $f<0$. This is not seen from the height of the peaks of the spectrum in Figure $7(\mathrm{~b})$ because the four-wave mixing signal has a broader linewidth than the regeneratively amplified signal. The broadening in the linewidth reduces the peak am litude in the measured spectrum. To make absolute comparison of the four-wave mixing and regeneratively amplified signals, the measured peak values have to be scaled by the respective linewidths. This has been pointed out and demonstrated by Hui and Mecozzi [Ref. 20]. In the spectra shown in Figure 7, the measured linewidth of the regeneratively amplified signal was limited by the $40 \mathrm{MHz}$ resolution of the scanning Fabry-Perot. We measured the linewidth of the four-wave mixing signal to be 1.6 times the instrument-limited linewidth of the regeneratively amplified signal. Thus, the true strength of the four-wave mixing signal in absolute comparison to that of the regenerative signal is the measured peak amplitude in Figure 7 multiplied by a factor 1.6. Figure 8 shows the spectra of the measured regeneratively amplified signal and four-wave mixing signal as functions of the detuning frequency $f$. The data of the four-wave mixing signal have been uniformly scaled by a factor 1.6 with respect to those of the regeneratively amplified signal to account for the difference in their linewidths. As can be seen, the spectrum of the four-wave mixing signal is symmetric. In contrast, the regeneratively amplified signal has an asymmetric spectrum. It is stronger than the four-wave mixing signal on the low-frequency side but is weaker on the highfrequency side, consistent with the prediction of Equation 26.

The data in Figure 8 were taken with the injected laser operating at a constant output power level of $7 \mathrm{~mW}$ and a constant optical injection level of $10 \mathrm{nW}$ while scanning the detuning frequency. At this injection level, the injection locking range was observed to be $<100 \mathrm{MHz}$ on both sides of the spectrum. Therefore, all the data were taken well outside the injection locking range. The corresponding power spectrum was taken simultaneously under the same operating conditions. The measured power spectrum as a function of the detuning frequency is shown in Figure 9. The power spectrum is symmetric with respect. to the zero detuning, as is expected from the discussion in the previous section. As is also shown in Figure 8, the optical spectrum data of 


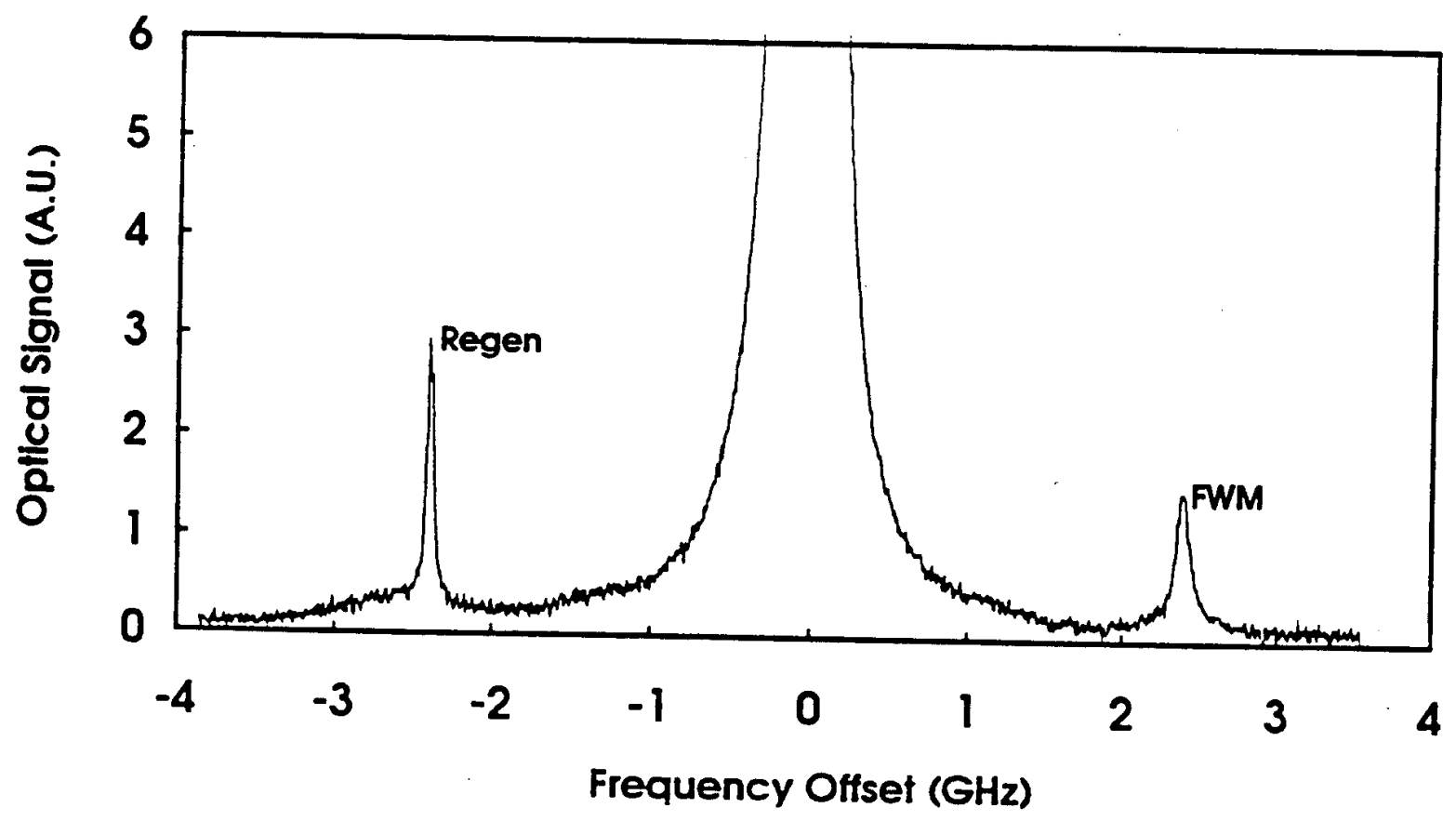

(a) Injection signal on low frequency side

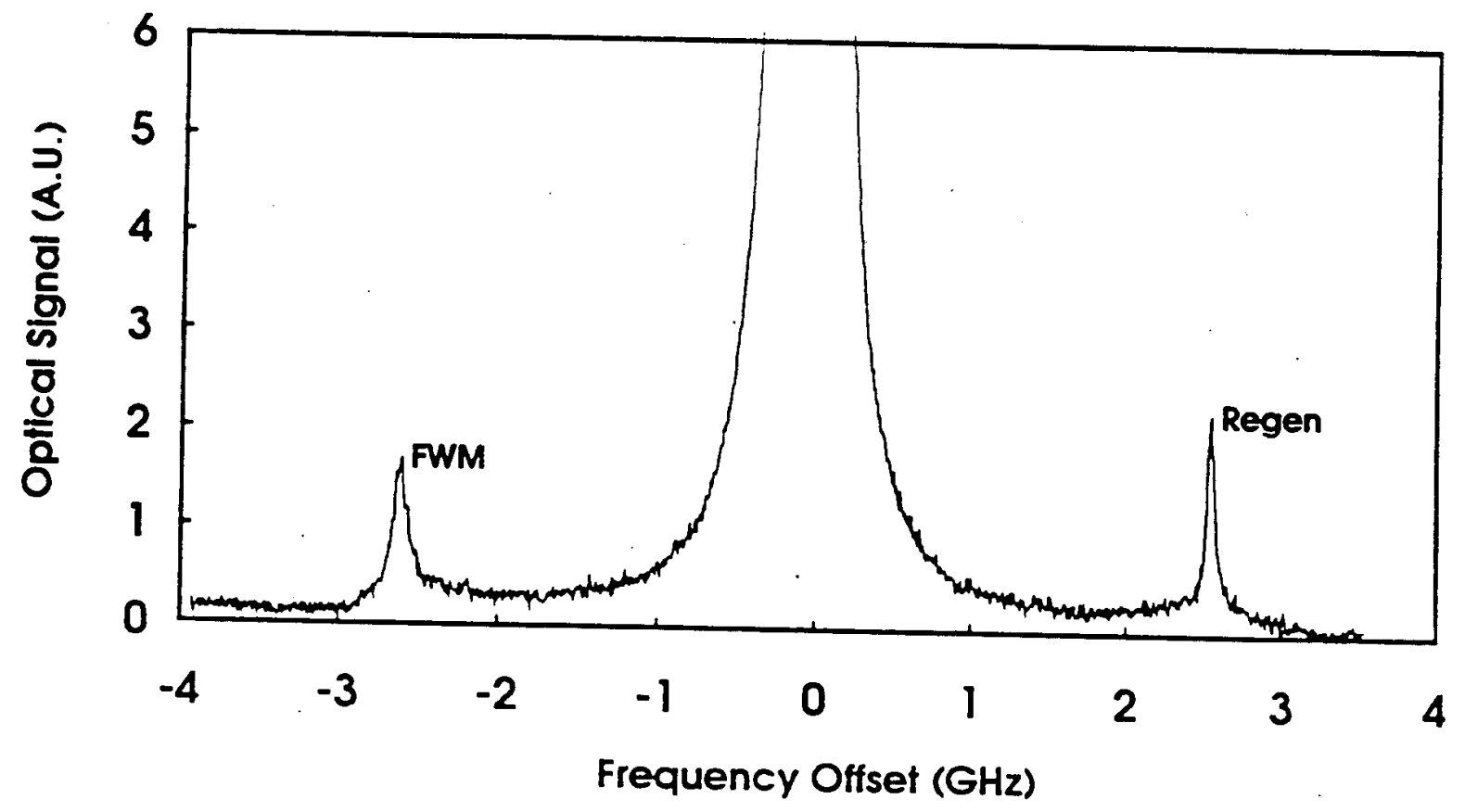

(b) Injection signal on high frequency side

Figure 7. Typical four-wave mixing spectra from the output of the injected laser. The injection signal is offset with respect to the oscillating frequency of the laser. 


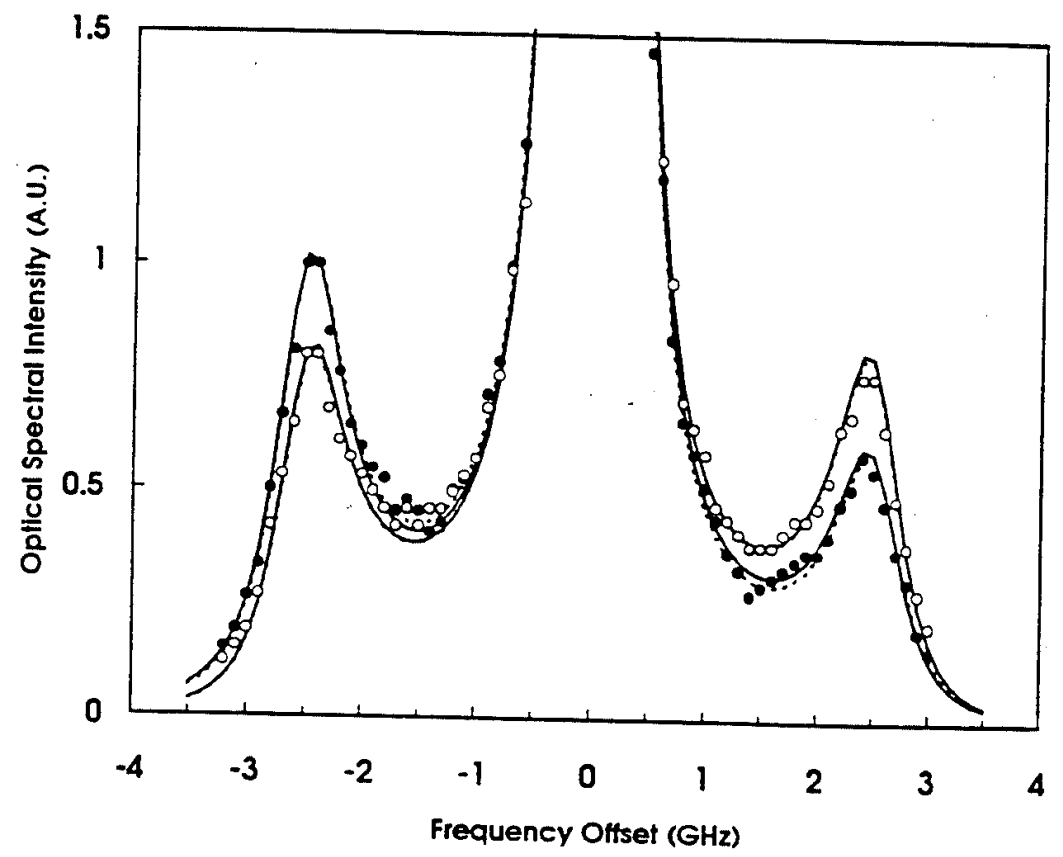

Figure 8. Experimentally measured and theoretically calculated regeneratively amplified and four-wave mixing signals from the optical spectral analysis as a function of the detuning frequency. The closed and open circles are the experimentally measured data for the regenerative and four-wave mixing signals, respectively. The solid and dotted curves are theoretical fits with $\gamma_{p}=2 \times 10^{9} \mathrm{~s}^{-1}$ and 0 , $b=3.5$. The injection locking used are $f_{r}=2.55 \mathrm{GHz}, \gamma_{r}=4.55 \times 10^{9} \mathrm{~s}^{-1}$, and $b=3.5$. The injection locking range is $<100 \mathrm{MHz}$ on both sides of the spectrum.

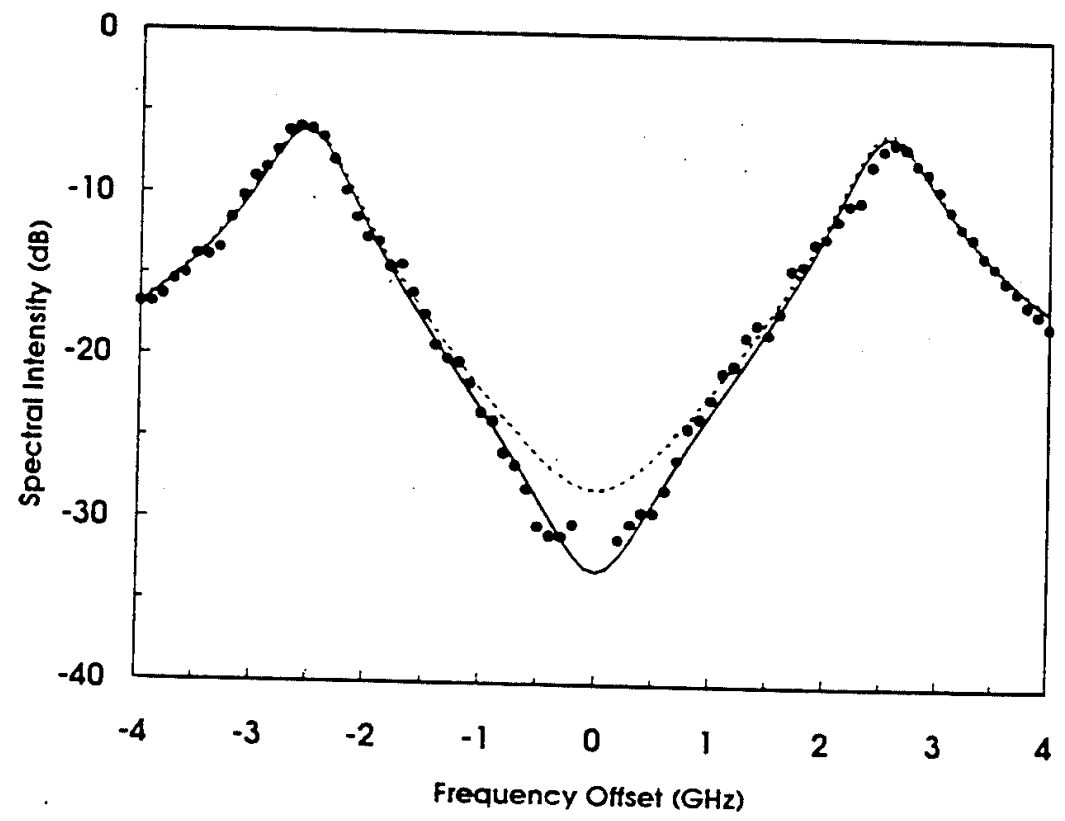

Figure 9. Experimentally measured and theoretically calculated power signal as a function of the detuning frequency. The closed circles are experimentally measured data. The solid and dotted curves are theoretical fits with $\gamma_{p}=2 \times 10^{9} \mathrm{~s}^{-1}$ and 0 , respectively. Other parameters used are $f_{r}=2.55 \mathrm{GHz}, \gamma_{r}=4.55 \times 10^{9} \mathrm{~s}^{-1}$, and
$\mathbf{b}=3.5$. The injection locking range is $<100 \mathrm{MHz}$ on both sides of the spectrum. 
both the regeneratively amplified and four-wave mixing signals can be fitted by the theoretically predicted spectra of $\left|A_{T}\right|^{2}$ and $\left|A_{f}\right|^{2}$ given by Equations 27 and 28, respectively. Similarly, the power spectrum data shown in Figure 9 can be fitted with $\sigma^{2}$ obtained from Equation 29. The data in Figures 8 and 9 are best fitted with $f_{r}=2.55 \mathrm{GHz}, \gamma_{r}=4.55 \times 10^{9} \mathrm{~s}^{-1}, \gamma_{p}=2 \times 10^{9} \mathrm{~s}^{-1}$, and $\mathrm{b}=3.5$.

\subsection{LASER PARAMETER CHARACTERIZATION}

From the previous discussions, it can be seen that the spectral data measured in a four-wave mixing experiment directly depend on the fundamental laser parameters of $f_{r}, \gamma_{r}, \gamma_{p}$, and $b$. In addition, other laser parameters, such as the carrier lifetime, $\tau_{s}$, and the photon lifetime, $\tau_{c}$, can be determined from the power dependencies of these parameters. Thus, the four-wave mixing experiment can be developed into a simple technique for accurate characterization of many intrinsic laser parameters [Ref. 21].

\subsubsection{Principle and Experimental Approach}

The optical spectra do not sensitively depend on $\gamma_{p}$. This is demonstrated in Figure 8 where the theoretical fits of the optical spectrum data with $\gamma_{p}=0$ and $\gamma_{p}=2 \times 10^{9} \mathrm{~s}^{-1}$ (but with the values of other parameters fixed) are compared. The same comparison is made for the power spectrum in Figure 9.

The power spectrum data cannot be fitted by a model without a nonlinear gain parameter, i.e., $\gamma_{p}=0$, but are best fitted by a nonlinear model with $\gamma_{p}=2 \times 10^{9} \mathrm{~s}^{-1}$ caused by gain saturation. The power spectrum is sensitive to the value of $\gamma_{p}$, particularly at small detuning frequencies less than $\pm 1 \mathrm{GHz}$. Thus, the power spectrum is needed for deducing the value of $\gamma_{p}$. In contrast, the value of $b$ can only be determined from the asymmetric optical spectrum of the regeneratively amplified signal. The power spectrum does not depend on the parameter $b$. Because $\left|A_{f}\right|^{2} \propto I+b^{2}$, as shown with Equation 28, the optical spectrum of the four-wave mixing signal is symmetric and its spectral shape does not depend on $b$, either. For the purpose of characterizing the laser parameters, it is thus only necessary to take both the optical spectrum. of the regeneratively amplified signal and the power spectrum. The optical spectrum of the fourwave mixing signal is not needed. 
Because it is not necessary to monitor the four-wave mixing signal, the scanning Fabry-Perot optical spectrum analyzer is not needed. The beam block B2 was inserted in the experimental setup shown in Figure 3. The spectral characteristics of the regeneratively amplified signal were measured by heterodyne detection with LD1 acting as local oscillator while the beam block B1 was removed from the optical path of $v_{l}$. This measures the regenerative reflectivity spectrum
given by

$$
\left|\frac{A_{r}}{A_{i}}\right|=\left|\frac{\eta}{\Omega}\right|\left[\frac{\left(\Omega^{2}-\Omega_{r}^{2} / 2-b \Omega \gamma_{p} / 2\right)^{2}+\left(\Omega \gamma_{r}-\Omega \gamma_{p} / 2-b \Omega_{r}^{2} / 2\right)^{2}}{\left(\Omega^{2}-\Omega_{r}^{2}\right)^{2}+\Omega^{2} \gamma_{r}^{2}}\right]^{1 / 2},
$$

which can be obtained from Equation 27. For this measurement, the spectrum analyzer was set at $80 \mathrm{MHz}$ with a bandwidth of $10 \mathrm{kHz}$ to record the beat signal generated by mixing of the outputs from both lasers. The power spectrum was measured by simply blocking the main output from LD1 by inserting the beam block B1 and allowing only the output of LD2 into the fast photodiode. This measurement records $\sigma_{2}$ given by

$$
\sigma^{2}=\left|\frac{\eta A_{i}}{A_{0}}\right|^{2} \frac{\Omega^{2}+\left(\gamma_{r}-\gamma_{p}\right)^{2}}{\left(\Omega^{2}-\Omega_{r}^{2}\right)^{2}+\Omega^{2} \gamma_{r}^{2}}
$$

In our experiment, $v_{O}$ of LD2 was held constant while a linear current ramp was repetitively applied to LD1. This swept $v_{1}$ for the detuning frequency $f$ to vary continuously at a constant rate over a range of negative and positive detuning, such as from -6 to $+6 \mathrm{GHz}$. Thus, both spectra were easily taken as functions of $f$. The spectra were then corrected for the slight change of the output power of LD1 caused by the linear current ramp.

\subsubsection{Procedure and Accuracy of Parameter Determination}

In using the spectral data to deduce the characteristic laser parameters, we first fit the regenerative reflectivity data with Equation 31 to determine the values of $f_{r}, \gamma_{r}$, and $b$. In this process, the value of $\gamma_{p}$ can be assumed to be zero, or any reasonable guess, without affecting the accuracy of the values of the other parameters. Once the values of $f_{r}, \gamma_{r}$, and $b$ are determined, $\gamma_{p}$ is the only free parameter left in fitting the power spectrum data with Equation 32 . It can thus be 
determined with very little uncertainty. In addition, the high-frequency portion of the power spectrum also provides a check on the values of $f_{r}$ and $\gamma_{r}$ determined from the regenerative reflectivity data. With this approach, the values of these parameters can be determined quite accurately without the knowledge of $\gamma_{c}$. This is an advantage over the existing techniques, which require a separate measurement of $\gamma_{c}$ before the value of $\gamma_{p}$ can be determined [Refs. 13, 16]. Indeed, the value of $\gamma_{c}$ can be determined from the power dependencies of $f_{r}, \gamma_{r}$, and $\gamma_{p}$.

A representative regenerative reflectivity spectrum taken with the heterodyne measurement is shown in Figure 10, together with the theoretical fitting curves using Equation 31 . The corresponding power spectrum is shown in Figure 11, together with the theoretical fitting curves using Equation 32. These data were taken when LD2 had a constant output power of $9.2 \mathrm{~mW}$ under a dc injection current of $40 \mathrm{~mA}$ and was injected with an injection signal of $\sim 20 \mathrm{nW}$. Again, the injection locking range was $<100 \mathrm{MHz}$ on both sides of the spectrum. The power spectrum is symmetric with respect to zero detuning; only one side of the spectrum is needed for the characterization of laser parameters. As can be seen, the values of $f_{r}=3 \mathrm{GHz}, \gamma_{r}=5.5 \mathrm{x}$ $10^{9} \mathrm{~s}^{-1}$, and $\mathrm{b}=3$ can be deduced from fitting the regenerative reflectivity spectral data without the knowledge of the value of $\gamma_{p}$. From the power spectrum, the value of $\gamma_{p}=2.6 \times 10^{9} \mathrm{~s}^{-1}$. can be determined. Figure 11 shows that the high-frequency portion of the power spectrum at $f>2 \mathrm{GHz}$ becomes very insensitive to the value of $\gamma_{p}$ but depends primarily on $f_{r}$ and $\gamma_{r}$. It is evident that the valus:s of $f_{r}$ and $\gamma_{r}$ determined from the regenerative reflectivity data are well checked out from the close fit between the theoretical curve and the experimental data of the power spectrum.

The parameter characterization procedure described has a very high degree of accuracy. The values of $f_{r}, \gamma_{r}$, and $\gamma_{p}$ can be determined to within 5 percent accuracy, but the value of $b$ is less accurate. As shown from Figure 11 and with Equation 32, using the parameters described above, the power spectrum peaks almost exactly at $f_{r}$, although the regenerative reflectivity spectrum peaks at a frequency about 98 percent that of $f_{r}$. Figure 12 demonstrates that a 10 percent change in the value of $\gamma_{r}$ creates substantial discrepancies between the theoretical fit and the experimental data in the regenerative reflectivity spectrum. Figure 13 shows that $b$ can be determined only to within an accuracy of plus or minus one. 


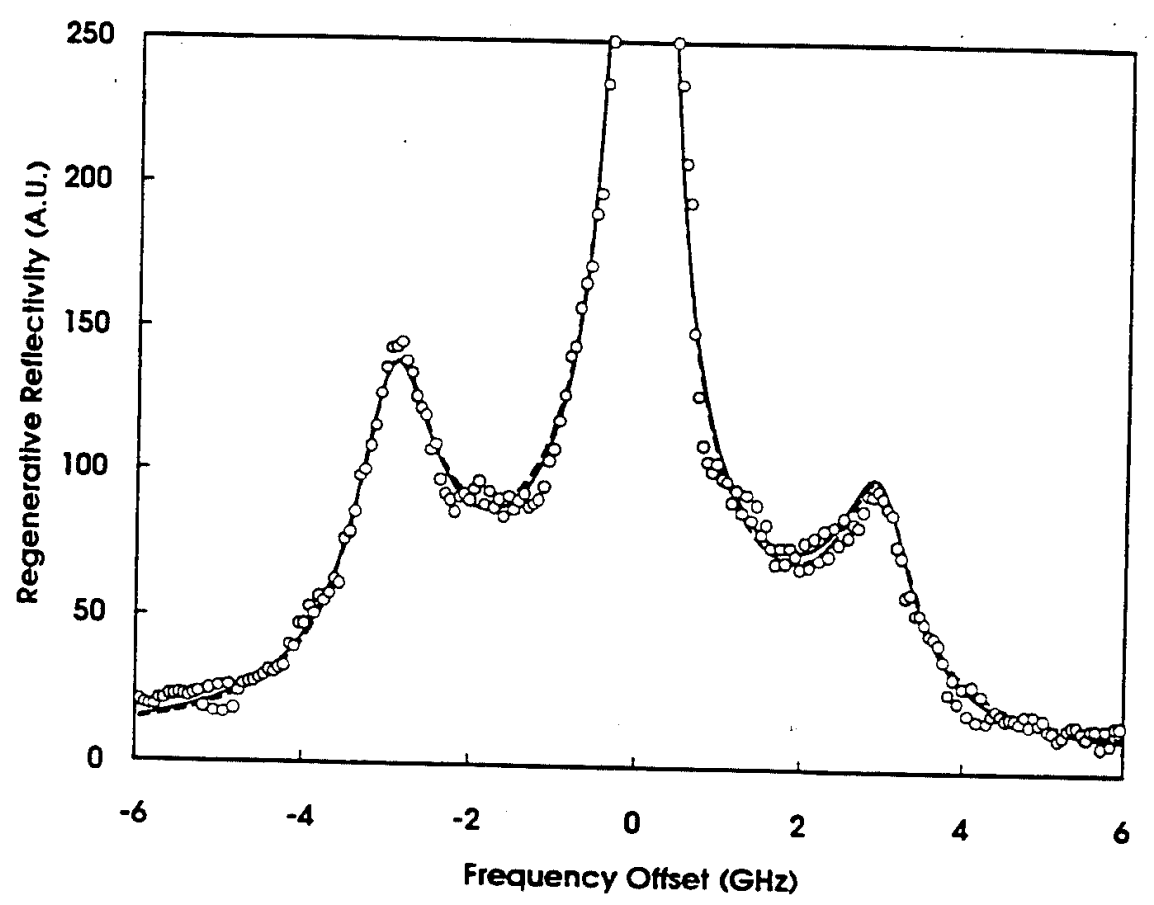

Figure 10. Representative spectrum of the regenerative reflectivity at a laser output power of $9.2 \mathrm{~mW}$. The open circles are experimental data taken with heterodyne measurement. The solid and dashed curves are theoretical fits with $\gamma_{p}=2.6 \times 10^{9} \mathrm{~s}^{-1}$ and 0 , respectively, but with fixed values of $f_{r}=3 \mathrm{GHz}, \gamma_{r}=5.5$ $x 10^{9} \mathrm{~s}^{-1}$, and $\mathrm{b}=3$. The injection locking range is $<100 \mathrm{MHz}$ on both sides of
the spectrum.

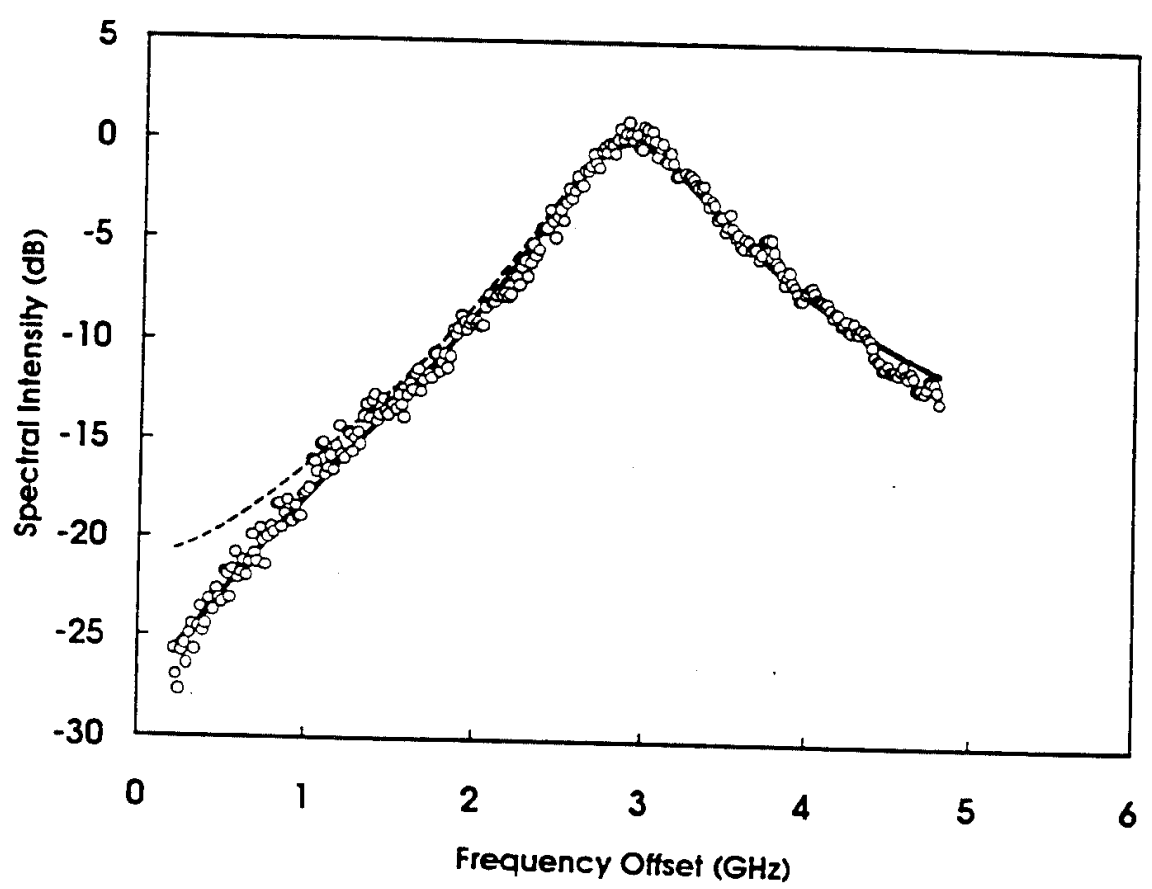

Figure 11. Representative power spectrum at a laser output power of $9.2 \mathrm{~mW}$. The open circles are experimental data. The solid and dashed curves are theoretical fits with $\gamma_{p}=2.6 \times 10^{9} \mathrm{~s}^{-1}$ and 0 , respectively, but with fixed values of $f_{r}=3 \mathrm{GHz}$, and $\gamma_{r}=5.5 \times 10^{9} \mathrm{~s}^{-1}$. The injection locking range is $<100 \mathrm{MHz}$. 


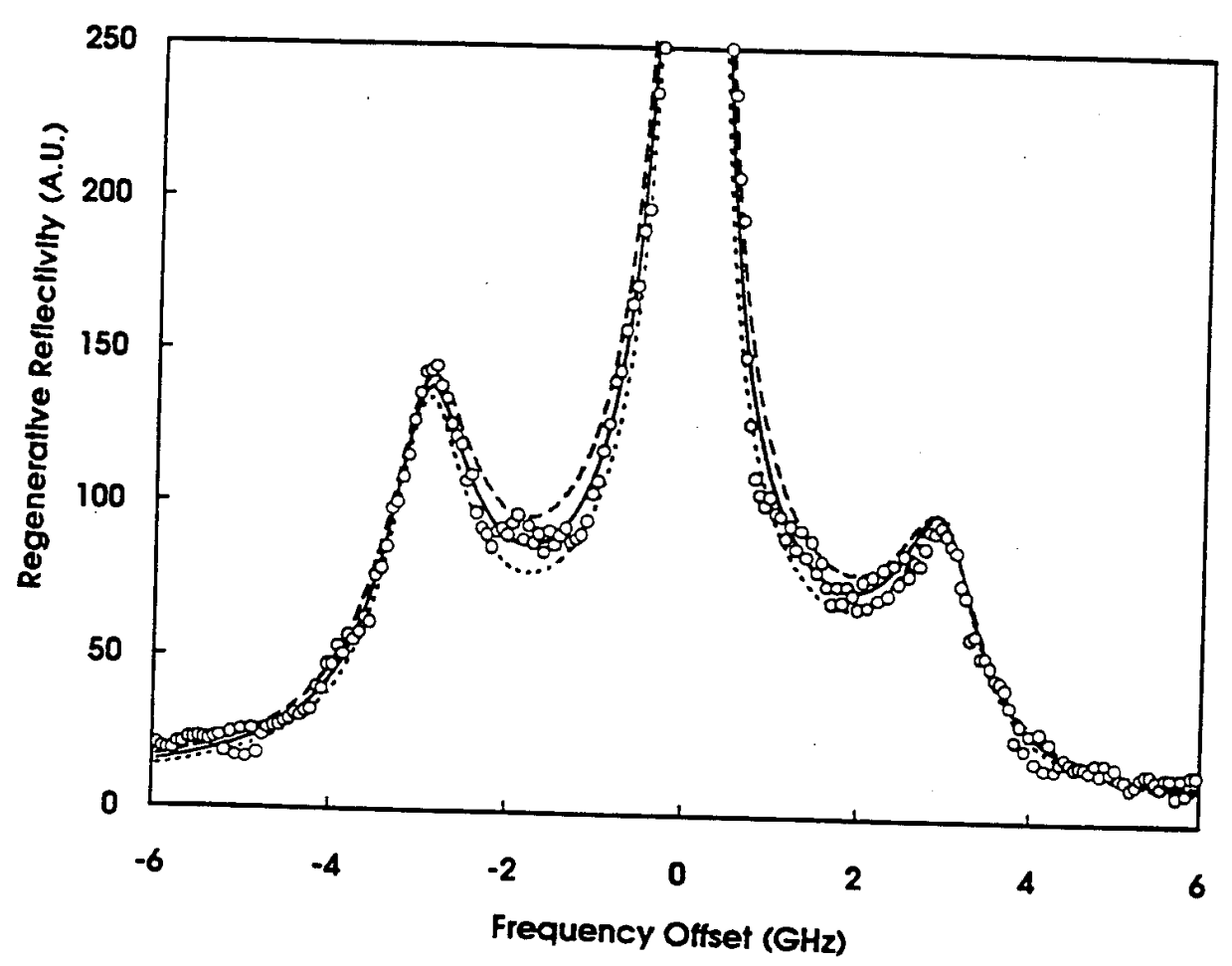

Figure 12. Sensitivity of the regenerative reflectivity spectrum to the value of $\gamma_{r}$. The open circles are experimental data. The dashed, solid, and dotted curves are theoretical fits with $\gamma_{r}=6,5.5$, and $5 \times 10^{9} \mathrm{~s}^{-1}$, respectively, but with fixed values of $f_{r}=3 \mathrm{GHz}, \gamma_{p}=2.6 \times 10^{9} \mathrm{~s}^{-1}$, and $b=3$.

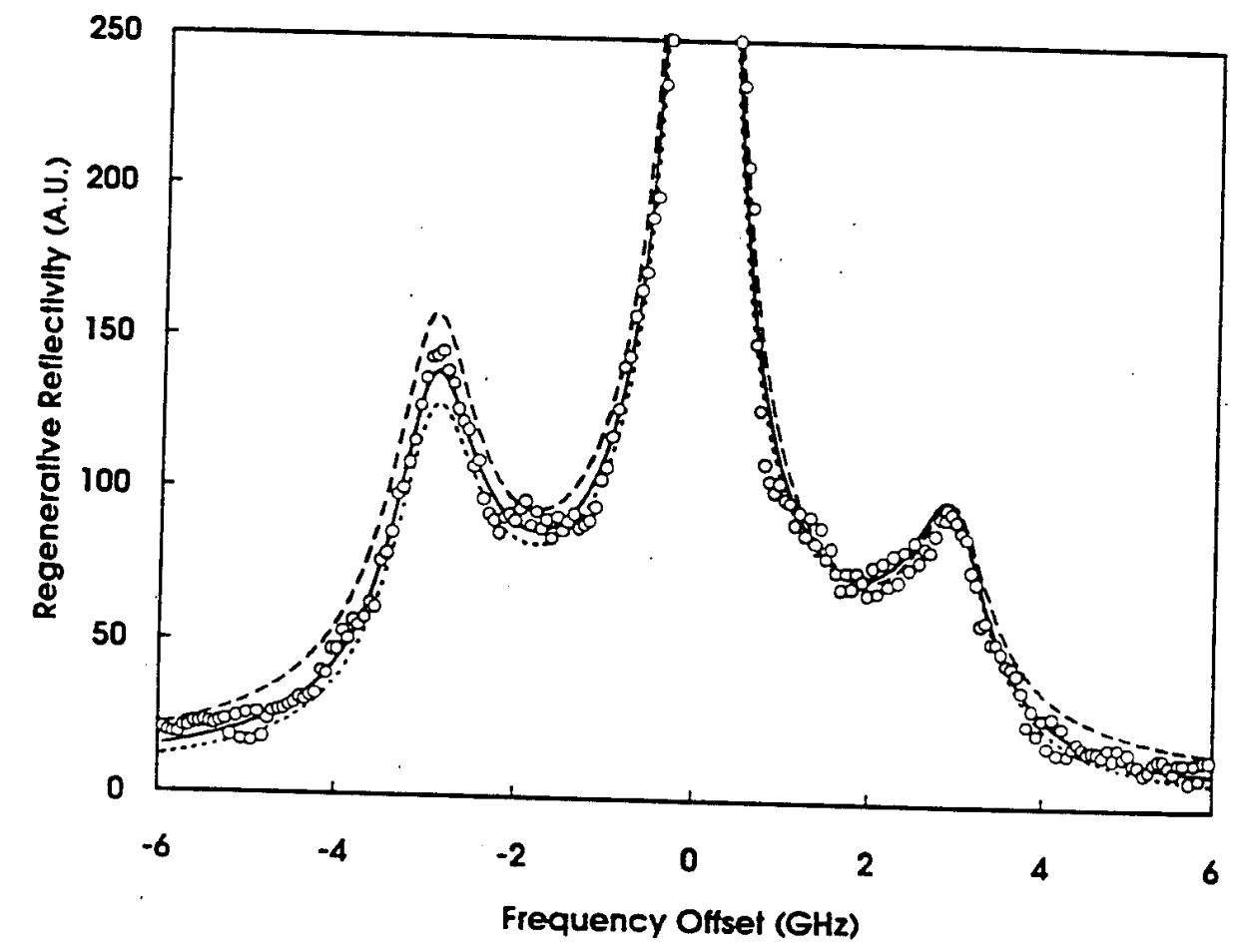

Figure 13. Sensitivity of the regenerative reflectivity spectrum to the value of $b$. The open circles are experimental data. The dashed, solid, and dotted curves are theoretical fits with $b=2,3$, and 4 , respectively, but with fixed values of $f_{r}=3 \mathrm{GHz}, \gamma_{r}=5.5 \times 10^{9} \mathrm{~s}^{-1}$, and $\gamma_{p}=2.6 \times 10^{9} \mathrm{~s}^{-1}$. 


\subsubsection{Power Dependence and Indirectly Deduced Parameters}

By taking a series of spectra at various levels of output power, $P$, of LD2, the power dependencies of the laser parameters can be obtained. Figure 14 shows the power dependence of the square of the resonance frequency, from which we obtain $f_{r}^{2}=0.954 P \mathrm{GHz}^{2}$, or $\Omega_{r}^{2}=37.66 P \mathrm{GHz}^{2}$, where $P$ is in milliwatts. The data in Figure 15 show that both $\gamma_{r}$ and $\gamma_{p}$ have a linear power dependence and are best described by $\gamma_{r}(1.458+0.435 P) \times 10^{9} \mathrm{~s}^{-1}$ and $\gamma_{p}=$ $0.28 \mathrm{P} \times 10^{9} \mathrm{~s}^{-1}$, respectively, where $P$ is in milliwatts. In contrast, the value of $b$ deduced from the regenerative reflectivity spectra taken at different power levels showed no power dependence and was found to be $b=3 \pm 1$. From the intercept at $P=0$ of the linear fitting of $\gamma_{r}$ shown in Figure 15 , a $\gamma_{s}=1.458 \times 10^{9} \mathrm{~s}^{-1}$, corresponding to a carrier lifetime of $\tau_{s}=686 \mathrm{ps}$, was obtained. Thus, from Equation 5, we have $\gamma_{n}=0.155 P \times 10^{9} \mathrm{~s}^{-1}$, where $P$ is in milliwatts. Using Equation 7 , the value of $\gamma_{c}=2.4 \times 10^{11} \mathrm{~s}^{-1}$ was deduced, which gives a photon lifetime of $\tau_{c}=4.2 \mathrm{ps}$. Let us assume that the output coupler is the dominant source of cavity losses, use the design cavity length of $500 \mu \mathrm{m}$, and assume an effective index of refraction of 3.5. We can then calculate that this cavity lifetime is associated with an output coupler reflectivity of 0.06 , which is similar to, but somewhat higher than, the design reflectivity of $\leq 04$ for this laser diode. Using the parameters of the laser structure, we have $S=6 \times 10^{13} P$, where $S$ is in inverse cubic centimeters and $P$ is in milliwatts. From the power dependencies of $\gamma_{n}$ and $\gamma_{p}$ and using $\Gamma=0.4$, the differential and nonlinear gain parameters can be determined from Equation 6 to be $g_{n}=2.6 \times 10^{-6} \mathrm{~cm}^{3} \mathrm{~s}^{-1}$ and $g_{p}=-1.18 \times 10^{-5} \mathrm{~cm}^{3} \mathrm{~s}^{-1}$, respectively. The $K$ factor of the laser is given by $K=\left(\gamma_{\mathrm{r}}-\gamma_{\mathrm{s}}\right) / f_{r}^{2}$ and its nonlinear component by $K_{n l}=\gamma_{p} / f_{r}^{2}$ [Ref. 16]. Using the data shown in Figures 14 and 15 , we obtain the values of $K=0.45 \mathrm{~ns}$ and $K_{n l}=0.29 \mathrm{~ns}$ for this laser. This laser was not designed for high-frequency applications.

Table 1 summarizes the parameters and their values. Also included in the table is the pumping parameter, $\tilde{J}$, which is the difference between the current required to achieve a given output power and the threshold current, normalized to the threshold current. This parameter is important in the full nonlinear analysis described later. Knowledge of the value of $\eta$ is not required in our parameter characterization procedure, $\eta$ always appears in combination with the external injection signal and, therefore, cannot be independently determined.

Before proceeding to a general discussion, we quantify the limits of applicability of the perturbation model we are using. This model makes the basic assumption that the injected signal does not significantly modify either the frequency or amplitude of the free-running peak of the semiconductor laser. Due to the inherent frequency jitter between our two lasers, we concentrated on measurements of the amplitudes of the central peak and the two side peaks. 


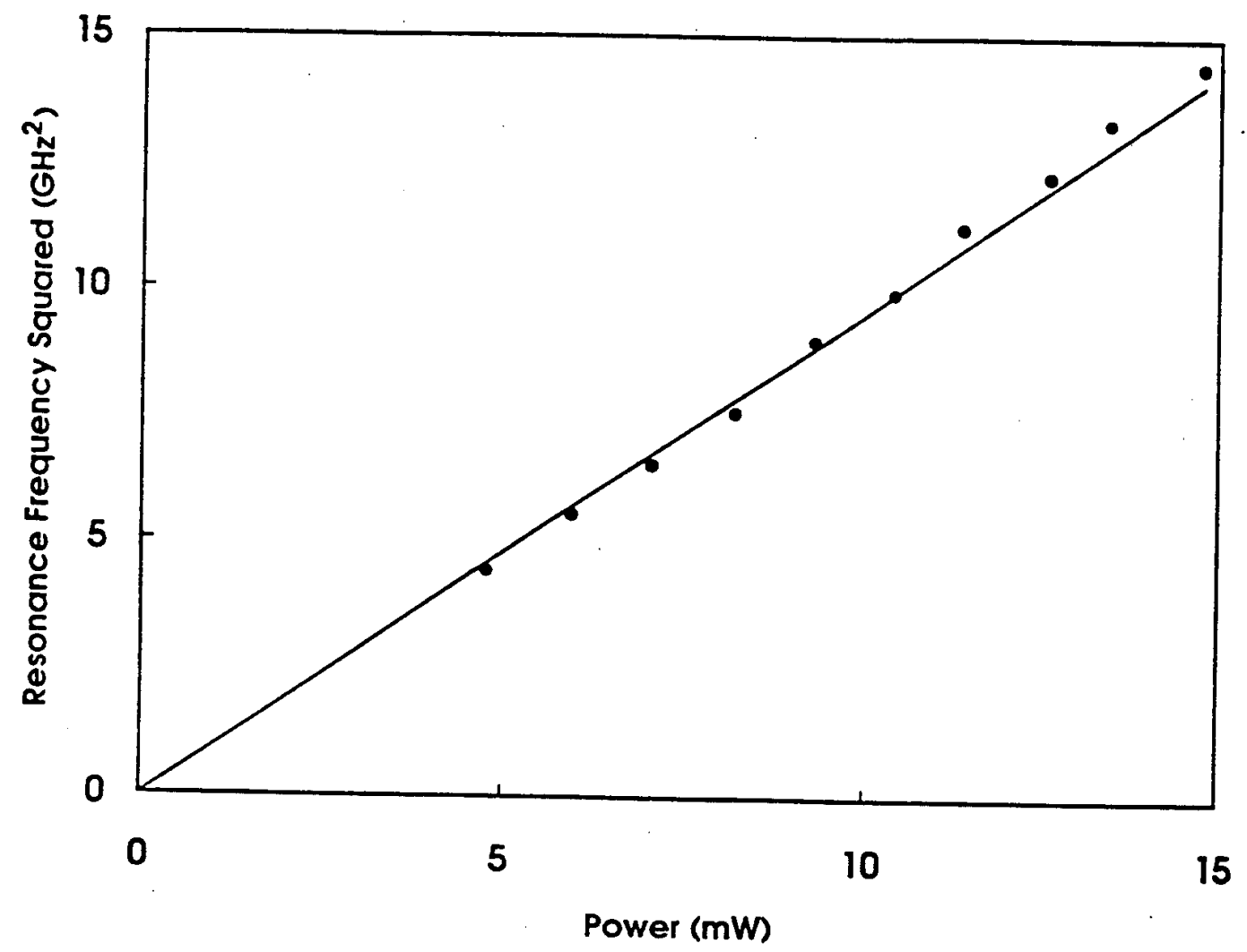

Figure 14. Power dependence of the square of the resonance frequency. The closed circles are experimental data and the solid line is the best linear fit.

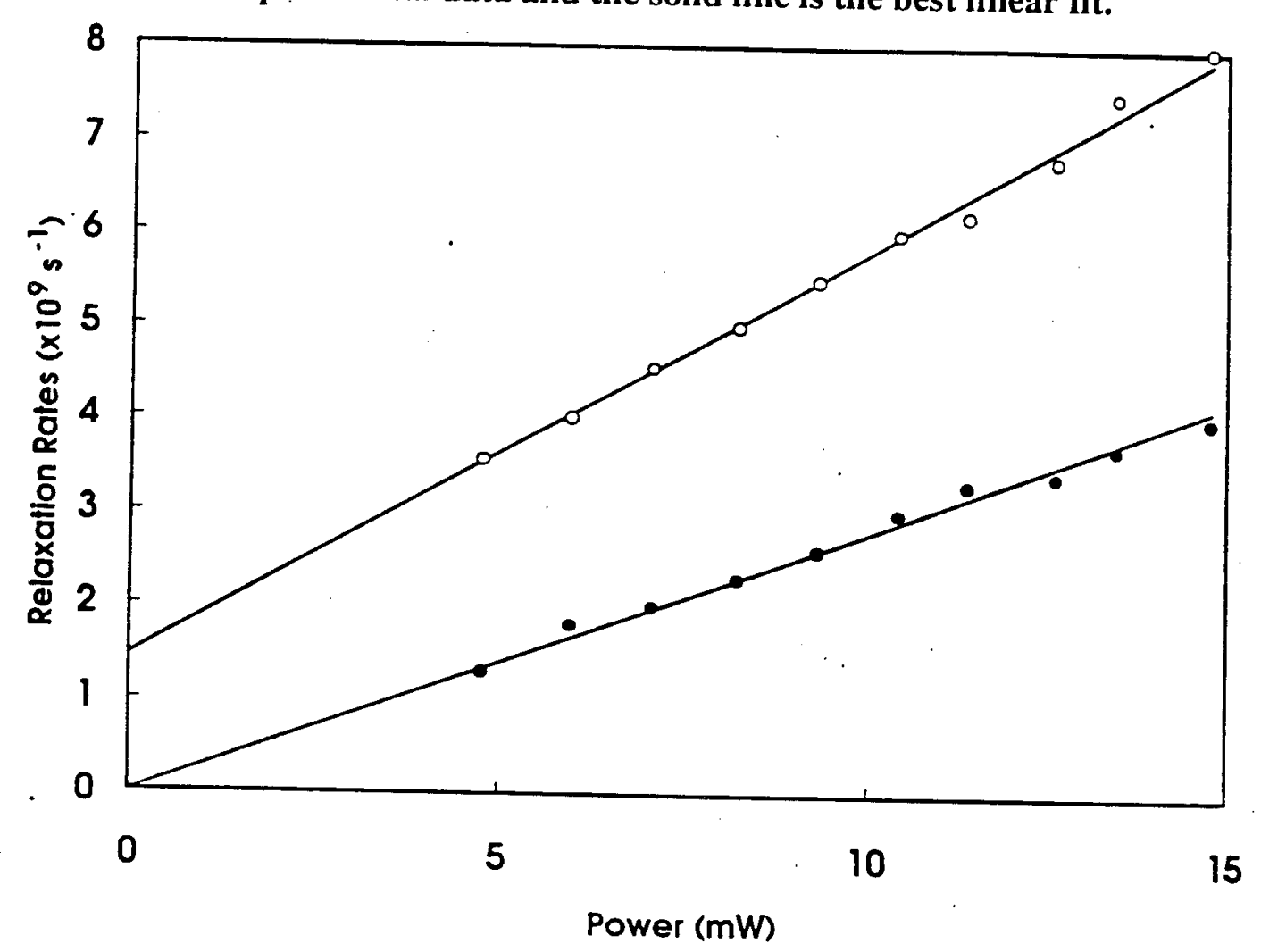

Figure 15. Power dependencies of $\gamma_{r}$, and $\gamma_{p}$. The open and closed circles are experimental data for $\gamma_{r}$ and $\gamma_{p}$, respectively. The solid lines are best linear fits. 


\begin{tabular}{|c|c|c|}
\hline Parameter & Symbol & Value* \\
\hline Laser Output Power & $P$ & 4 to $15 \mathrm{~mW}$ \\
\hline Pump Parameter & $\tilde{J}$ & $0.078 P$ \\
\hline Intracavity Photon Density & $S$ & $6 \times 10^{13} P \mathrm{~cm}^{-3}$ \\
\hline Confinement Factor & $\Gamma$ & 0.4 \\
\hline Injection Coupling Rate & $\eta$ & $2 \times 10^{11} \mathrm{~s}^{-1}$ \\
\hline Linewidth Enhancement Factor & $b$ & $3 \pm 1$ \\
\hline Relaxation Resonance Frequency & $f_{r}$ & $(0.954 P)^{1 / 2} \mathrm{GHz}$ \\
\hline Relation Resonance Angular Frequency & $\Omega_{r}$ & $(37.66 P)^{1 / 2} \times 10^{9} \mathrm{~s}^{-1}$ \\
\hline Total Carrier Relaxation Rate & $\gamma_{r}$ & $(1.458+0.435 P) \times 10^{9} \mathrm{~s}^{-1}$ \\
\hline Spontaneous Carrier Relaxation Rate & $\gamma_{s}$ & $1.458 \times 10^{9} \mathrm{~s}^{-1}$ \\
\hline Spontaneous Carrier Lifetime & $\tau_{s}$ & $686 \mathrm{ps}$ \\
\hline Differential Carrier Relaxation Rate & $\gamma_{n}$ & $0.155 \mathrm{P} \times 10^{9} \mathrm{~s}^{-1}$ \\
\hline Nonlinear Carrier Relaxation Rate & $\gamma_{p}$ & $0.28 \mathrm{P} \times 10^{9} \mathrm{~s}^{-1}$ \\
\hline Cavity Decay Rate & $\gamma_{c}$ & $2.4 \times 10^{11} \mathrm{~s}^{-1}$ \\
\hline Photon Lifetime & $\tau_{c}$ & $4.2 \mathrm{ps}$ \\
\hline Differential Gain Parameter & $g_{n}$ & $2.6 \times 10^{-6} \mathrm{~cm}^{3} \mathrm{~s}^{-1}$ \\
\hline Nonlinear Gain Parameter & $g_{p}$ & $-1.18 \times 10^{-5} \mathrm{~cm}^{3} \mathrm{~s}^{-1}$ \\
\hline$K$ factor & $K$ & $0.45 \mathrm{~ns}$ \\
\hline Nonlinear $K$ factor & $K_{n l}$ & $0.29 \mathrm{~ns}$ \\
\hline
\end{tabular}

* The laser power, $P$, in the values of the power-dependent parameters is in milliwatts.

Figure 16 plots the central peak, regenerative amplification, and four-wave mixing signals as a function of the injection signal. All signals are normalized to the free-running central peak value; the injection signal was detuned to the low frequency relaxation-resonance peak. The injection parameter, $\xi$, is defined as $\left(\eta\left|A_{i}\right| / \gamma_{c}\left|A_{0}\right|\right)$ and is calibrated by taking the measurement at $\xi \approx 8 \times 10^{-4}$ as the reference point and using Equations 27 and 28 . The figure shows depletion of the central peak and deviation of the nonlinear mixing signals when the signal strength is $\geq 0.02$ of the central peak. All measurements were below this limit. 


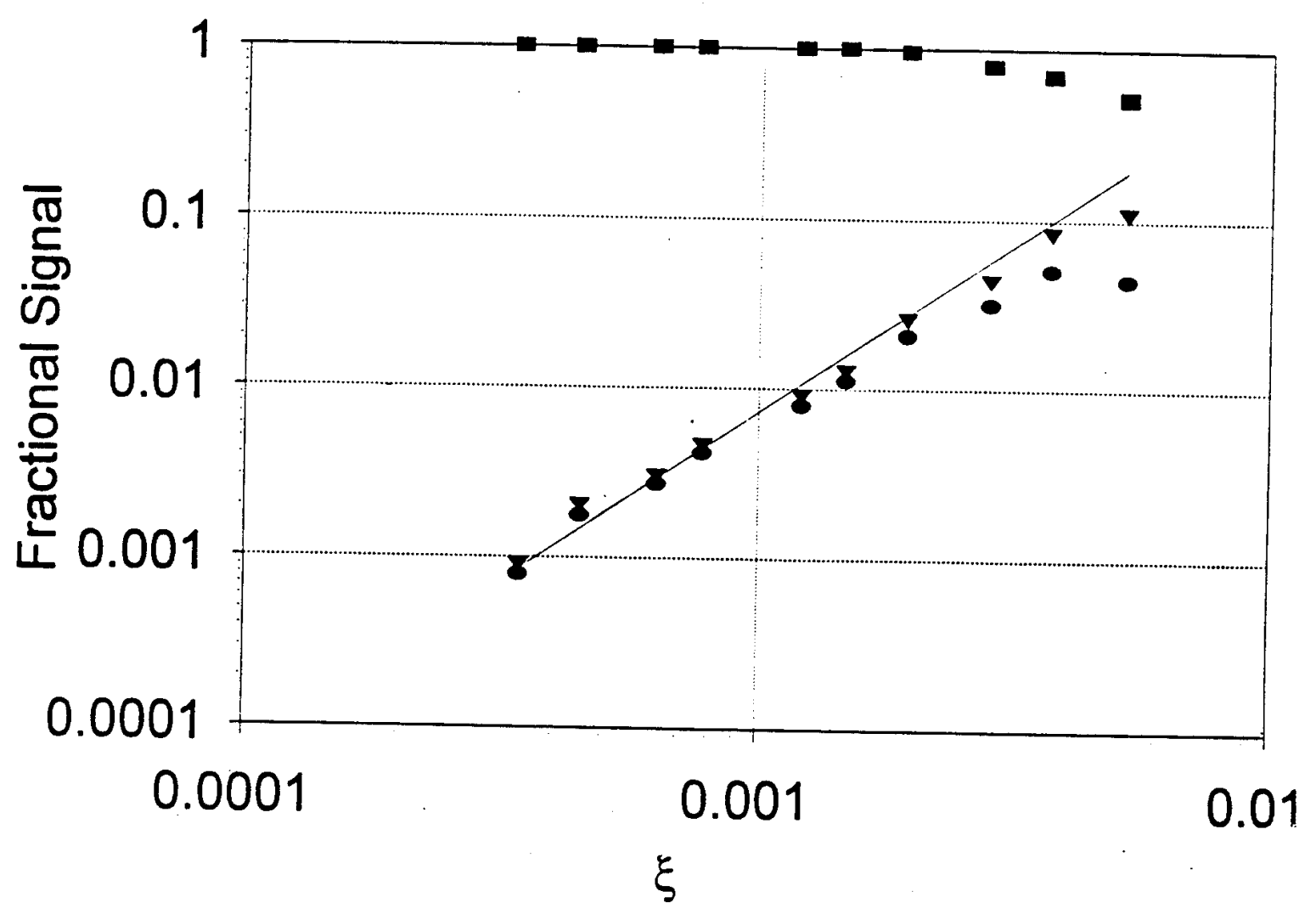

Figure 16. Log-log plot of the measured values of features in the optical spectra as a function of the normalized injection, $\xi$. The squares, triangles, and ovals correspond to measured values, normalized to the free-running central peak signal, for the central peak, regenerative-amplification sideband and four-wave mixing sideband, respectively. The solid line gives the slope expected in the weak injection limit for the two sidebands.

\subsection{DISCUSSION}

Nearly degenerate four-wave mixing in a semiconductor laser with the injection signal outside of the injection-locking range can be understood in terms of optical modulation in the field of the injected laser. Both the regeneratively amplified and the four-wave mixing signals have contributions from the amplitude and phase modulations of the laser field. The phase and amplitude characteristics of the optically modulated laser diode cannot be recovered with a model using the traveling-wave formalism which emphasizes the spatial interaction. Instead, a model of an unlocked, optically injected laser is used to emphasize the phase and amplitude constraints imposed by the optical resonator. This results in a thorough understanding of the spectral characteristics of the interaction, as well as the physical origins of the symmetry or asymmetry of each spectrum. It also correctly connects the spectral characteristics to the intrinsic parameters of the semiconductor laser oscillator. 
The dependence of the spectral characteristics on the laser parameters can be used as a simple and accurate technique for characterizing the intrinsic laser parameters. This technique is parasitic-free. It is only necessary to take the regenerative reflectivity spectrum and the power spectrum. We showed that the power spectrum, which measures the amplitude modulation and has usually been ignored in the previous four-wave mixing experiments reported in the literature, is needed for characterizing the nonlinear relaxation rate. The fundamental laser parameters of relaxation resonance frequency, total relaxation rate, nonlinear relaxation rate, and linewidth enhancement factor are determined from the spectra data obtained from a single experimental setup. Other parameters, including the spontaneous carrier lifetime, the photon lifetime, the differential and nonlinear gain parameters, and the $K$ factor, are determined from the power dependencies of these parameters. This technique depends on the understanding of the relation between four-wave mixing and optical modulation in the lase: and is possible only after this connection is made. It requires only two cw lasers closely matched in wavelength. It is applicable to semiconductor lasers of any wavelength and any dynamic bandwidth. 


\subsection{NOISE IN A SEMICONDUCTOR LASER}

Though there has been a great deal of work examining the noise spectra of laser diodes, there has not been fully consistent, quantitative agreement between theory and experimental data. In a recent combined experimental and theoretical study, it was concluded that the observed field noise spectra could be reproduced with a model of a strictly single-mode laser diode with spontaneous emission into the mode supplying the only noise source [Ref. 13]. In this data fitting, however, the values of several parameters had to be determined from the same data without independent verification, and the amplitude, i.e., intensity noise, spectra were not measured. Other experimental studies have indicated that even weakly emitting side modes of a nearly single-mode laser diode can have a significant influence on the amplitude spectrum of the oscillating mode [Refs. 22, 23]. This noise source, called mode partition noise, has been previously analyzed in the limit of one strong mode [Refs. 24, 25, 26]. These calculations indicated that the amplitude noise spectrum of the oscillating mode is greatly enhanced, relative to the single-mode case. However, they predicted that the overall amplitude noise from the output of all modes would be similar to that from a strictly single-mode laser diode whose only noise source is spontaneous emission into the oscillating mode.

Here, we show how a laser diode's noise spectra, away from line center, can be related to the modulation spectra generated by weak external optical injection, away from the injection-locking region [Refs. 19, 21]. The dynamic parameters of the laser diode required for a description of the weak external injection are also required to correctly model the noise spectra. Also, it is necessary to measure both the optical field and amplitude spectra in both cases. An external optical source modulates the diode output through the nonlinear optical coupling between the injected signal and the oscillating mode. The noise spectra generated by spontaneous emission into the oscillating mode will have the same shape as the combined regenerative-amplification and four-wave mixing spectra induced by optical injection. Using our previous results on the phase and amplitude properties of the modulation induced by optical injection [Ref. 21], we observe significant deviation of the noise spectra from this combined spectra. The deviations can be explained by including a colored noise term in the carrier density rate equation. Finally, we show that the source of this colored noise term is the amplified spontaneous emission of the weak side modes of the laser. Our results demonstrate that the nonlinear coupling between the principal oscillating mode and amplified spontaneous emission into both the principal mode and the weak side modes is the dominant source of noise in a nearly single-mode laser diode. 
As with the weak optical injection results described in the last section, we will be using a perturbation analysis of the noise spectra. Therefore, we restrict our attention to frequencies offset by at least $200 \mathrm{MHz}$, well outside of the $\approx 10 \mathrm{MHz}$ linewidth of the laser diode.

Typical field and amplitude spectral data are shown in Figures 17 and 18, respectively. The spectra show side peaks which are known to occur at approximately the relaxation resonance frequency [Ref. 8]. These side peaks are more than $30 \mathrm{~dB}$ below the central peak in the field spectra. A key feature of the field spectra is the asymmetry of the side peaks, which is also observed in the regenerative amplification spectra of an optically injected signal [Ref. 19]. In the latter case, however, the relative difference between the side-peak signals is more than twice as large as that in the noise spectra. At offset frequencies below $\sim 1.5 \mathrm{GHz}$, there is a strong difference between the amplitude spectra of the principal mois and the total diode output. Above $1.5 \mathrm{GHz}$ the two sets of data overlap so that only one is shown in Figure 18. The principal mode has an amplitude noise spectra which strongly increases with decreasing offset frequency, while the amplitude noise from all modes continues to decrease.

In Section 5.0, we performed a linearized analysis of the coupled Equations 1 and 2, by using the solution, Equation 9, for the regenerative amplification and four-wave mixing components. For the analysis of the noise spectra, we use an equivalent linearization procedure which more closely follows past work [Refs. 10,13]. Equations 1 and 2 are linearized, the injection term is set to zero, and the complex field equation is separated into two real equations for the amplitude and phase. It is convenient to use $A(t)=A_{0}[l+a(t)] e^{i \phi(t)}$ and $N(t)=N_{O}[1+\tilde{n}(t)]$. After separating out the time-independent terms that are the solution with the noise source term set to zero, the linearized equations can be written as

$$
\begin{aligned}
& \frac{d a}{d t}=\frac{\gamma_{c} \gamma_{n}}{2 \gamma_{s} \tilde{J}} \tilde{n}-\gamma_{p} a+\frac{F_{\text {real }}}{A_{0}} \\
& \frac{d \phi}{d t}=-b\left(\frac{\gamma_{c} \gamma_{n}}{2 \gamma_{s} \tilde{J}} \tilde{n}-\gamma_{p} a\right)+\frac{F_{i m a g}}{A_{0}} \\
& \frac{d \tilde{n}}{d t}=-\left(\gamma_{s}+\gamma_{n}\right) \tilde{n}-2 \gamma_{s} \tilde{J}\left(1-\frac{\gamma_{p}}{\gamma_{c}}\right) a+F_{c}
\end{aligned}
$$




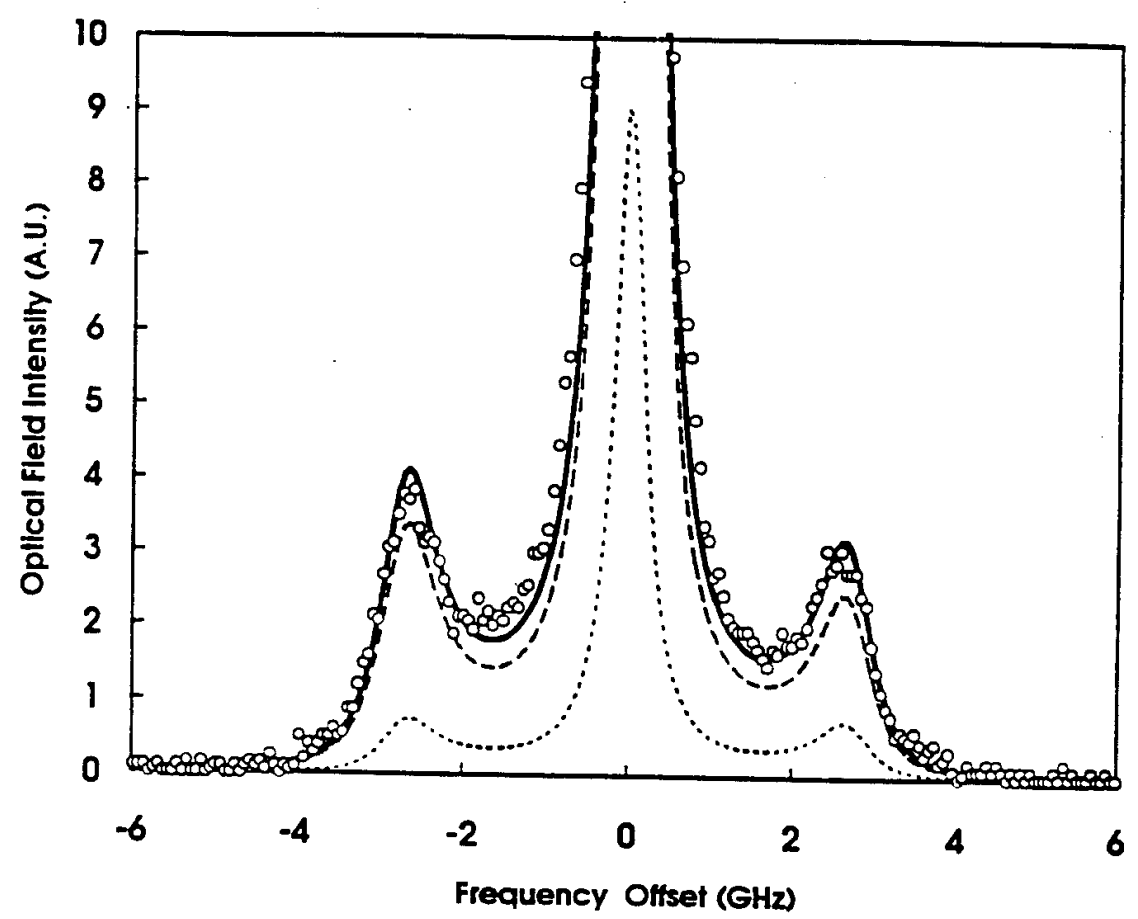

(a) Output power is $8 \mathrm{~mW}$

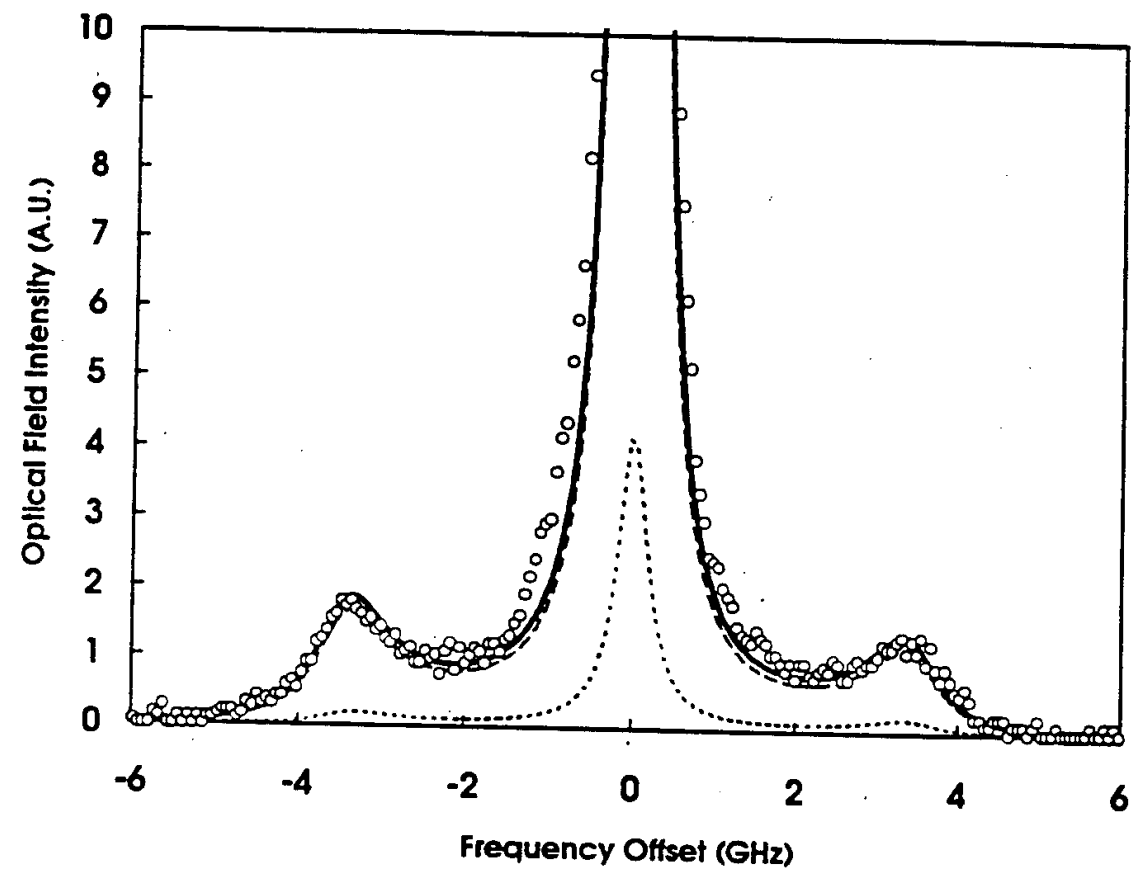

(b) Output power is $12.5 \mathrm{~mW}$

Figure 17. Experimentally measured and theoretically calculated field spectra of the laser diode principal mode. The open circles are experimentally measured data. The dashed and dotted curves are model calculations of the field and effective carrier noise contributions, respectively. The thick solid curve is the sum of
the two. 


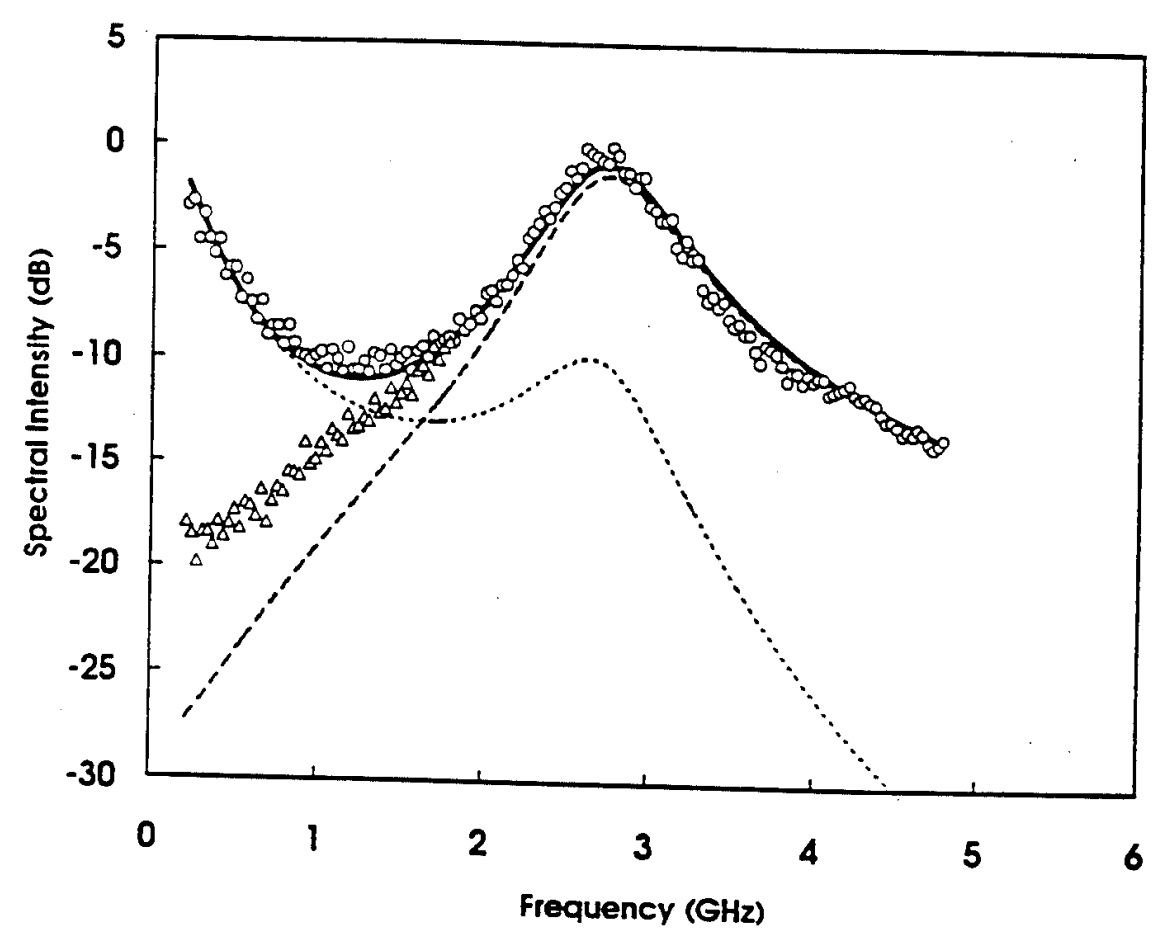

(a) Output power is $8 \mathrm{~mW}$

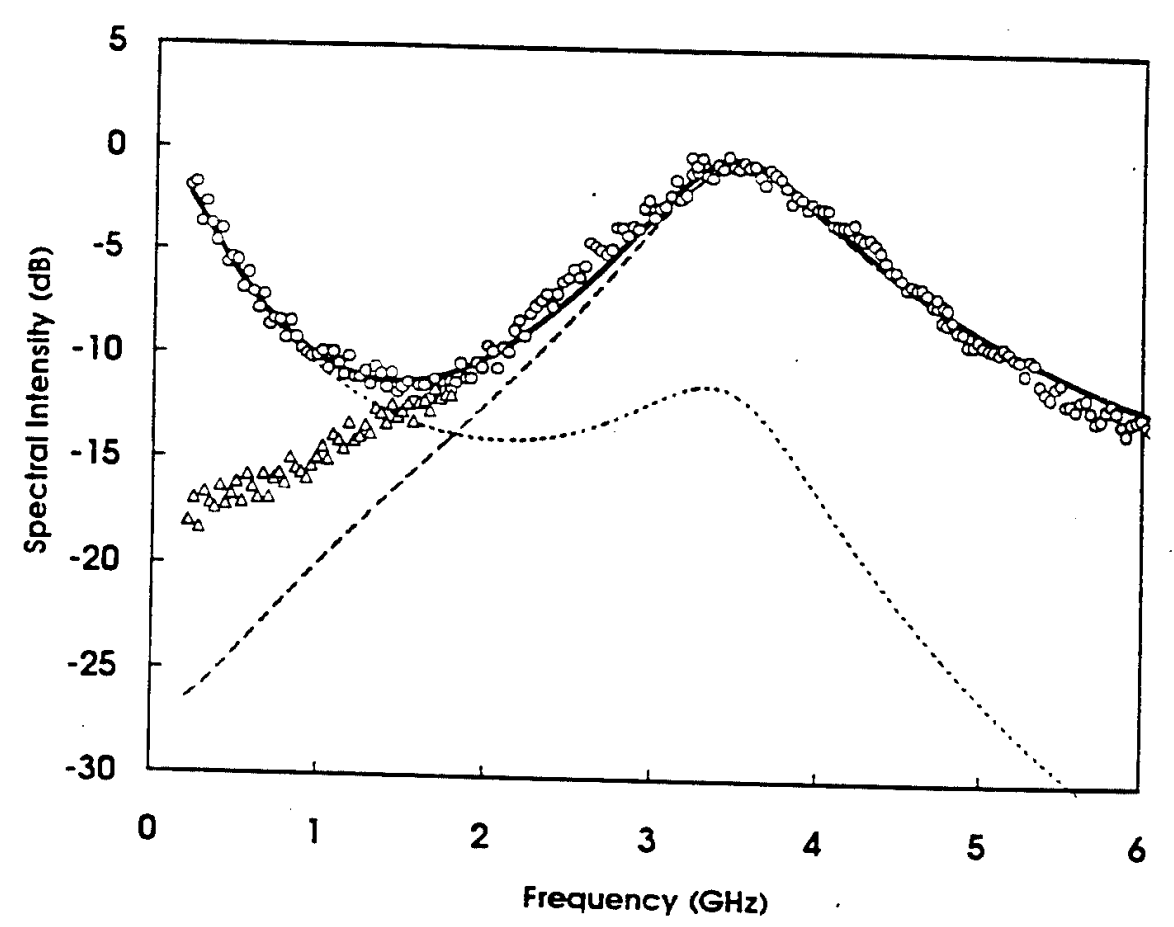

(b) Output power is $12.5 \mathrm{~mW}$

Figure 18. Experimentally measured and theoretically calculated amplitude spectra. The circles are experimentally measured data taken with an etalon passing only the principal mode. The triangles are data taken with the etalon removed. The dashed and dotted curves are model calculations of the field and effective carrier noise contributions, respectively. The thick solid curve is the 
Most of the parameters have been previously defined. Their values, determined by the four-wave mixing technique, are summarized in Table 1. The complex Langevin source term of Equation 1, $F_{s p}$, is divided into $F_{s p}=F_{\text {real }}+i F_{\text {imag. }}$. The real effective carrier noise is $F_{c}$. Spontaneous emission is the cause of the field noise and has been previously shown to have uncorrelated real and imaginary components and an effective delta-function self correlation in time [Ref. 10]. The spontaneous emission noise components satisfy

$$
\begin{aligned}
& \left\langle F_{\text {real }}(t) F_{\text {real }}\left(t^{\prime}\right)\right\rangle=\left\langle F_{\text {imag }}(t) F_{\text {imag }}\left(t^{\prime}\right)\right\rangle=\frac{R_{s p}}{2} \delta\left(t-t^{\prime}\right) \\
& \left\langle F_{\text {real }}(t) F_{\text {imag }}\left(t^{\prime}\right)\right\rangle=0
\end{aligned}
$$

where $R_{s p}$ gives the rate of spontaneous emission into the mode. The characteristics of $F_{c}$ will be discussed in greater detail next.

The Fourier transforms of the set of equations are used to find the resulting spectra. The field spectra are related to the Fourier transform of $[a(t)+i \phi(t)]$ and the amplitude spectra are related to the Fourier transform of $a(t)$. This procedure is equivalent to saying that the noise terms only weakly perturb the system which breaks down for Fourier components whose offset frequency is very close to the $\mathrm{cw}$, steady-state oscillating frequency. Separating the terms into the field noise source generated term, $A_{s p}$, and the carrier noise source generated term, $A_{c}$, by assuming that the carrier noise source is uncorrelated with the spontaneous emission into the oscillating mode, we find

$$
\begin{gathered}
\left|A_{s p}\right|^{2}=\frac{\left(\Omega_{r}^{4}+\Omega^{2} \gamma_{p}^{2}\right)\left(1+b^{2}\right) / 2+\left[\Omega^{2}-\Omega_{r}^{2}+\gamma_{r}\left(\gamma_{s}+\gamma_{n}\right)\right] \Omega_{2}-b\left[\gamma_{p}\left(\Omega^{2}-\Omega_{r}^{2}\right)+\gamma_{r} \Omega_{r}^{2}\right] \Omega}{\Omega^{2}\left[\left(\Omega^{2}-\Omega_{r}^{2}\right)^{2}+\Omega^{2} \gamma_{r}^{2}\right]} \frac{R_{s p}}{2} \\
\left|A_{c}\right|^{2}=\frac{\Gamma^{2} g_{n}^{2} A_{O}^{2}\left(1+b^{2}\right) / 4}{\left(\Omega^{2}-\Omega_{r}^{2}\right)^{2}+\Omega^{2} \gamma_{r}^{2}}\left\langle\digamma_{c}^{*}(\Omega) \digamma_{c}(\Omega)\right\rangle
\end{gathered}
$$

where $F_{\mathrm{c}}$ is the Fourier transform of $F_{c}$. The field noise generates an asymmetric spectra due to the linewidth enhancement factor, $b$. 
Also, as with external optical injection, the noise-induced fluctuations can be considered as amplitude and phase modulations of the coherent signal. The amplitude modulation coefficient, $\sigma$, for the two noise sources will have the spectra

$$
\begin{aligned}
& \sigma_{s p}^{2}=\frac{\Omega^{2}+\left(\gamma_{s}+\gamma_{n}\right)^{2}}{\left(\Omega^{2}-\Omega_{r}^{2}\right)^{2}+\Omega^{2} \gamma_{r}^{2}} \frac{R_{s p}}{2 A_{O}^{2}}, \\
& \sigma_{c}^{2}=\frac{\Gamma^{2} g_{n}^{2} / 4}{\left(\Omega^{2}-\Omega_{r}^{2}\right)^{2}+\Omega^{2} \gamma_{r}^{2}}\left\langle F_{c}^{*}(\Omega) \digamma_{c}(\Omega)\right\rangle .
\end{aligned}
$$

The field-noise contribution to the amplitude spectra, $\sigma_{s p}^{2}$, is identical to that derived for optical injection by an external source [Ref. 19]. In the field spectrum, its contribution, Equation 38, is identical to the sum of the regenerative amplification and four-wave mixing signals that occur under optical injection [Ref. 19]. This is because the spontaneous emission is, effectively, a weak external optical source in the approximations of this model. By comparison with external optical injection, the noise spectra due to spontaneous emission arise from the nonlinear optical interaction between the principal mode and the injected spontaneous signal.

Because the characteristic parameters of the laser have been independently determined using an external optical source, we are constrained in our choice of parameters to fit the measured spectra. The only free parameters are the relative contributions from $F$ and $F_{c}$ and the spectral shape of $F_{c}$. As can be seen from the dashed curves in Figures 17 and 18, the measured data cannot be explained by considering only the spontaneous emission noise in the principal mode. Careful analysis of the amplitude noise data shows that the additional noise in the principal mode can be fit with a carrier noise source with a simple Lorentzian frequency profile, $\left\langle F_{c}^{*}(\Omega) F_{c}(\Omega)\right\rangle \propto\left(\Omega^{2}+Z^{2}\right)^{-1}$, where $\mathrm{Z} / 2 \pi$ must be between 200 to $300 \mathrm{MHz}$ to simultaneously fit both the amplitude and field data.

Carrier shot noise would have a white source spectrum, like the spontaneous emission noise, and the noise spectra it produces are calculated to be too weak and of the wrong spectral shape to account for the measured spectra. We have also determined that the carrier noise does not have its source in the current source or bias circuit characteristics of the laser diode. However, the noise strongly depends on the partition of laser power among the modes. By examining several 
laser diodes with various levels of side mode emission, we observed a direct correlation between the relative power in the side modes and the strength of the low frequency noise on the principal mode.

We have measured the total power in the weak side modes to be $<10$ percent of the total output. Therefore, the side modes will have only a small impact on the saturation parameter, $\gamma_{p}$. The place in the coupled equations where the sides modes will have the strongest influence is in the carrier density equation. Equation 2 becomes

$$
\frac{d N}{d t}=\frac{J}{e d}-\frac{N}{\tau_{s}}-\frac{2 \varepsilon_{0} n^{2}}{\hbar \omega_{0}}\left(g|A|^{2}+\sum_{\kappa} g_{\kappa}\left|A_{\kappa}\right|^{2}\right)
$$

when the side modes are explicitly included. The subscript $\kappa$ labels the various side modes. The weak side modes generate an effective carrier noise term, $F_{c}$ in Equation 35 , with a bandwidth determined by the linewidth of the side modes. This can be seen by considering that, now, each of the side modes must be modeled by an equation similar to Equation 1. The complex amplitude for the side modes can be separated into amplitude and phase components, $A_{K}(t)=A_{\kappa}\left[1+a_{\kappa}(t)\right] e^{i \phi_{\kappa}(t)}$. The stronger side modes have a measured power between 0.1 and 1 percent of the principal mode power. The linearized equations for the amplitude spectra of these side modes can be simplified to

$$
\frac{d a_{\kappa}}{d t}=-\delta g_{\kappa} a_{\kappa}+\frac{F_{\kappa, \text { real }}}{A_{\kappa O}}
$$

where $\delta g_{\kappa}=g-g_{\kappa}$, the difference between the gain of the principal mode and the side mode $\kappa$, and $F_{K}$ is the spontaneous emission source term for that mode. When these equations are Fourier transformed, they yield the appropriate noise source term $\left(F_{C}\right)$ in the carrier equation if the linewidths of the side modes, given by $\delta g_{\kappa}$, can be approximated by an effective average value of $Z / 2 \pi$.

Limited by the tuning range of the second laser, we have measured the field linewidth of two of the stronger side modes of the laser using the optical-injection technique. One spectrum is shown in Figure 19, along with a Lorentzian curve fit assuming $Z / 2 \pi=220 \mathrm{MHz}$. The other mode had a similar linewidth. Though the effective linewidth from all of the modes is not necessarily the same as the linewidth of the modes measured, these should be representative. We have used $220 \mathrm{MHz}$ in the calculated curves in Figures 17 and 18. The data fit is excellent. 


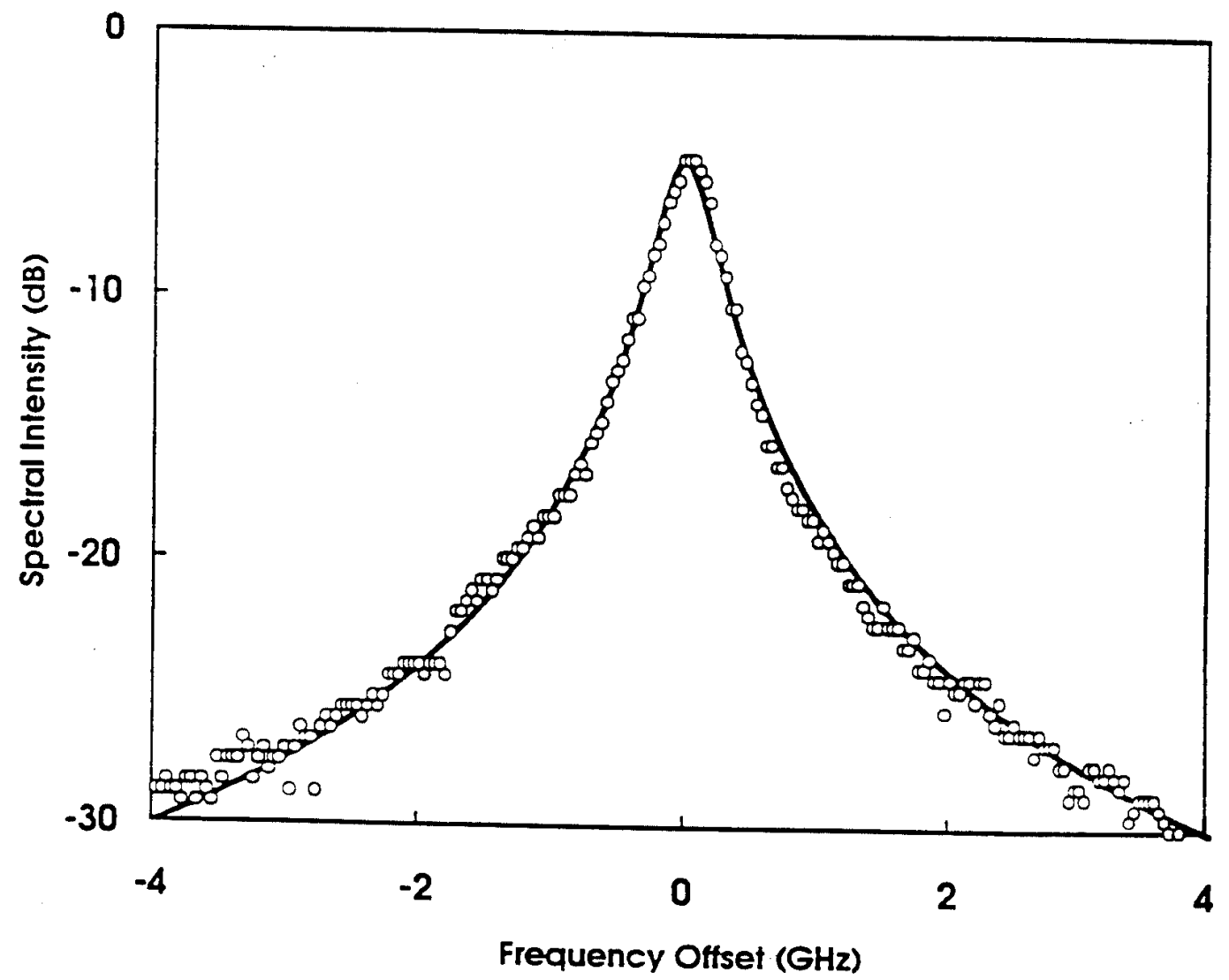

Figure 19. Experimentally measured side mode field spectrum (open circles) and Lorentzian curve fit (solid curve) with a half-width half-maximum
linewidth of $220 \mathrm{MHz}$.

We observe residual additional noise in the amplitude spectrum, above the spontaneous emission model curve, when the total diode output is measured. Again, a carrier white-noise source has the wrong spectral shape to simultaneously fit both optical field and amplitude spectra. Side modes described by Equations 42 and 43, and other models [Refs. 25, 27], also do not account for this noise due to the negative correlation between the total amplitude and carrier fluctuations in models with a homogeneous gain spectrum [Ref. 27]. Very weak side modes which are not well correlated with the principal mode, due to inhomogeneous effects in the gain spectrum, can account for the additional noise [Refs. 28, 29].

In our model we have ignored noise source terms in the carrier density equation that do not arise from field source terms. Some of these terms are correlated with the spontaneous emission field noise term [Refs. 9, 30, 31]. Including these terms in the carrier equation is the way that spontaneous-emission noise is differentiated from external noise sources. In the so-called shotnoise-limited regime, these sources can have a significant impact on the type of spectra we measure [Refs. 30,31]. These noise terms do not significantly contribute to the spectra that we 
measure because we are operating far from this noise limit. This is because we are operating at relatively low pump parameters, $0.25 \leq \tilde{J} \leq 1$, and due to the presence of the poorly correlated side modes. Under these operating conditions, the field noise components generally dominate the carrier noise components due to the shape of the spectra.

In our measurements, the nonlinear gain in the principal mode and the mixing between the spontaneous emission into the mode and the oscillating field account for the dominant noise contribution to the field spectrum. This noise is augmented by mode partition noise, which is much more important in the amplitude spectrum. While the mode partition noise enters into the single-mode model as a carrier noise source term, it is important to remember that the side mode emission is amplified spontaneous emission. The principal mode sees a fluctuating refractive index due to the fluctuating side mode intensities. Therefore, the nonlinear optical interaction of the principal mode field scattering off of the fluctuating refractive index generated by the amplified spontaneous emission into the side modes creates the additional noise. 


\subsection{RESONANT INJECTION IN THE STRONG SIGNAL REGIME}

\subsection{INTRODUCTION}

Chaotic dynamics in optical systems is the subject of considerable current research [Refs. 32, 33]. Laser diodes have attracted considerable interest, not only for their wide range of applications but, also, for the study of fundamental laser dynamics. Due to their small size and large gain, free-running semiconductor lasers have an optical spectrum that is largely determined by the fundamental spontaneous emission noise into the oscillating mode [Ref. 9]. Like a variety of laser systems, an isolated, dc-biased, single-mode semiconductor laser is sufficiently described by only two rate equations (Class B lasers); one for the photon density and another for the carrier density [Refs. 10,12]. Consequently, while this system can show nonlinear oscillatory dynamics, it cannot exhibit chaotic behavior. To induce chaos in such a system, a third degree of freedom (such as external optical injection to cross-couple the phase and amplitude of the oscillating field or pump modulation to induce additional dynamics in the gain medium) is needed [Ref. 12]. Early theoretical and experimental investigations of lasers under external optical injection showed a variety of unstable dynamic phenomena but did not consider a range of parameters relevant to semiconductor laser diodes [Refs. 12, 34-36]. It has been predicted more recently that external optical injection into a laser diode can lead to chaos through the period-doubling route, though the experimental evidence has been ambiguous [Ref. 37]. Laser diodes provide a technologically important system to study both high-speed nonlinear dynamics and fundamental noise processes in lasers. Further, they can be used to test the utility of laser dynamics models in the strongly nonlinear regime. However, because of the very short time scales (subnanosecond) on which the dynamics occurs, it is difficult to measure the timedependent behavior directly. Fourier measurements must be combined with model calculations to extract information about the properties of the nonlinear dynamics in these fast dynamical systems [Refs. 37-39].

To perform numerical simulations using the coupled-equation model, we use a set of three real equations in a form which explicitly shows the dependence on specific laser parameters which can be experimentally determined in the weak injection limit [Ref. 38]:

$$
\frac{d a}{d t}=\frac{1}{2}\left[\frac{\gamma_{c} \gamma_{n}}{\gamma_{S} \tilde{J}} \tilde{n}-\gamma_{p}\left(2 a+a^{2}\right)\right](1+a)+\eta a_{i} \cos (\Omega t+\phi)+F^{\prime} /\left|A_{O}\right| \text {. }
$$




$$
\begin{aligned}
& \frac{d \phi}{d t}=-\frac{b}{2}\left[\frac{\gamma_{c} \gamma_{n}}{\gamma_{s} \tilde{J}} \tilde{n}-\gamma_{p}\left(2 a+a^{2}\right)\right]-\frac{\eta a_{i} \sin (\Omega t+\phi)-F^{\prime \prime}|| A_{0} \mid}{1+a}, \\
& \frac{d \tilde{n}}{d t}=-\gamma_{s} \tilde{n}-\gamma_{n}(1+a)^{2} \tilde{n}-\gamma_{s} \tilde{J}\left(2 a+a^{2}\right)+\frac{\gamma_{s} \gamma_{p}}{\gamma_{c}} \tilde{J}\left(2 a+a^{2}\right)(1+a)^{2} .
\end{aligned}
$$

Here, $\mathrm{a}=\left(|A|-\left|A_{0}\right|\right) /\left|A_{O}\right|$ and $a_{i}=\left|A_{i}\right| /\left|A_{0}\right|$, where $|A|$ is the amplitude of the slave laser oscillating field, $\left|A_{0}\right|$ is the free-running, steady-state field amplitude, and $\left|A_{i}\right|$ is the amplitude of the injection field. The frequency offset of the injection laser from the free-running frequency of the slave laser is $\Omega$ and $\phi$ is the phase difference between $A$ and $A_{i}$. In $\tilde{n}=\left(N-N_{O}\right) / N_{O}, N$ is the carrier density and $N_{O}$ is the steady-state carrier density of the free-running laser. Langevin source terms for spontaneous emission noise injected into the laser mode are $F^{\prime}$ and $F^{\prime \prime}$ [Refs. 10, 40]. To be explicit, $\left(F^{\prime}=F_{\text {real }} \cos \phi-F_{\text {imag }} \sin \phi\right)$ and $\left(F^{\prime \prime}=F_{\text {real }} \sin \phi+\right.$ $\left.F_{\text {imag }} \cos \phi\right)$. The dependence of $F^{\prime}$ and $F^{\prime \prime}$ on $\phi$ can be accounted for in the rapid uncorrelated nature of the noise source fluctuations. Other parameters are defined in Section 3.0. All input parameters required to numerically solve the set of coupled differential equations for $a, \phi$, and $\tilde{n}$ can be experimentally determined. Equations 33 through 35 are the linearized versions of Equations 44 through 46 with $a_{i}=0$.

The coupled equations were numerically integrated, and the resulting time series were Fourier transformed, for various injection levels. Noise and dynamic parameters of the laser were independently determined by the four-wave mixing experiment [Refs. 21,40$]$, and $\Omega$ was set equal to zero to model optical injection at the free-running frequency. In the experiments, the master laser was tuned to the free-running oscillation frequency of the slave laser. Residual offsets, due to frequency jitter between the two lasers, were $<100 \mathrm{MHz}$. The slave laser is injection locked, but the dynamics are not necessarily stable under such injection [Ref. 41]. The master laser's central linewidth was significantly less than the linewidth of the free-running slave laser. Further, at all but the highest injection levels considered here, its noise spectra, away from the central peak, was significantly weaker than the spontaneous emission by the slave laser into its oscillating mode.

To determine the injection level, we calibrated the system by measuring the four-wave mixing spectrum in the weak injection limit and used our previously developed model of the interaction to compare the generated sideband signal with the central peak [Refs. 19, 21]. The normalized injection level is $\xi=\left(\eta\left|A_{i}\right|\right) /\left(\gamma_{c}\left|A_{0}\right|\right)$. The injected power is proportional to $\xi^{2}$. 


\subsection{PERIOD-DOUBLING ROUTE TO CHAOS}

First, we present experimental evidence that strong optical injection into a laser diode from a master laser operating at the free-running oscillation frequency of the laser diode is sufficient to drive it into a chaotic regime through a period-doubling route. Experimentally observed features are demonstrated using a single-mode injection model [Ref. 19] which includes spontaneous emission noise [Ref. 40]. All parameters required for the model are listed in Table 1, allowing a quantitative comparison between experimentally measured spectra and the predictions of the model. We have found that the transition to chaos can be used to reduce the uncertainty in the measured value of the linewidth enhancement factor of the laser diode.

Figure 20 shows two typical spectra of the principal mode of the slave laser. The frequency is relative to the free-running frequency or, equivalently, the injection frequency. In Figure 20(a), $\xi=1.7 \times 10^{-2}$ and $\xi=2.6 \times 10^{-2}$ in Figure 20(d). When taking these spectra, the slave laser was operated at an output level of $9 \mathrm{~mW}$, where its free-running relaxation resonance frequency is $f_{r}=2.9 \mathrm{GHz}$. When the optical injection is increased beyond the weak injection limit for stable injection locking, it first causes unstable oscillation of the laser at the resonance frequency. The relatively narrow peaks in the spectrum of Figure 20(a) are separated by a frequency spacing of $f_{r}$ and are typical features of highly unstable injection locking [Ref. 41]. For injection levels with $\xi \leqslant 1.2 \times 10^{-2}$, the optical spectrum contains very little output except in these narrow peaks. As the injection level is further increased, broad features appear in the spectrum between the narrow oscillation peaks, as is seen in Figure 20(a). At even higher injection levels, the spectrum becomes dominated by a broad pedestal and many strong secondary peaks develop (Figure 20(d)). At this stage, chaos is fully developed, as will be demonstrated by comparison with calculated spectra. We have also observed that as the laser is driven into the chaotic region, the average oscillating power in the principal mode decreases by as much as 36 percent while the overall output is approximately constant. The power is spread among several of the weak side modes.

Two sets of calculated optical spectra were obtained at injection levels similar to the corresponding experimental spectra shown in Figure 20. The spectra in Figures 20(b) and (e) are calculated with no spontaneous emission noise term in the field equations, while the spectra in Figures 20(c) and (f) include a spontaneous emission noise source. The positions of the computed peaks appear at the same frequencies as the corresponding experimental peaks. Relative strengths of the computed peaks are consistent with the experimentally obtained spectra when noise is present, though there is some discrepancy in the details of the different peaks. 


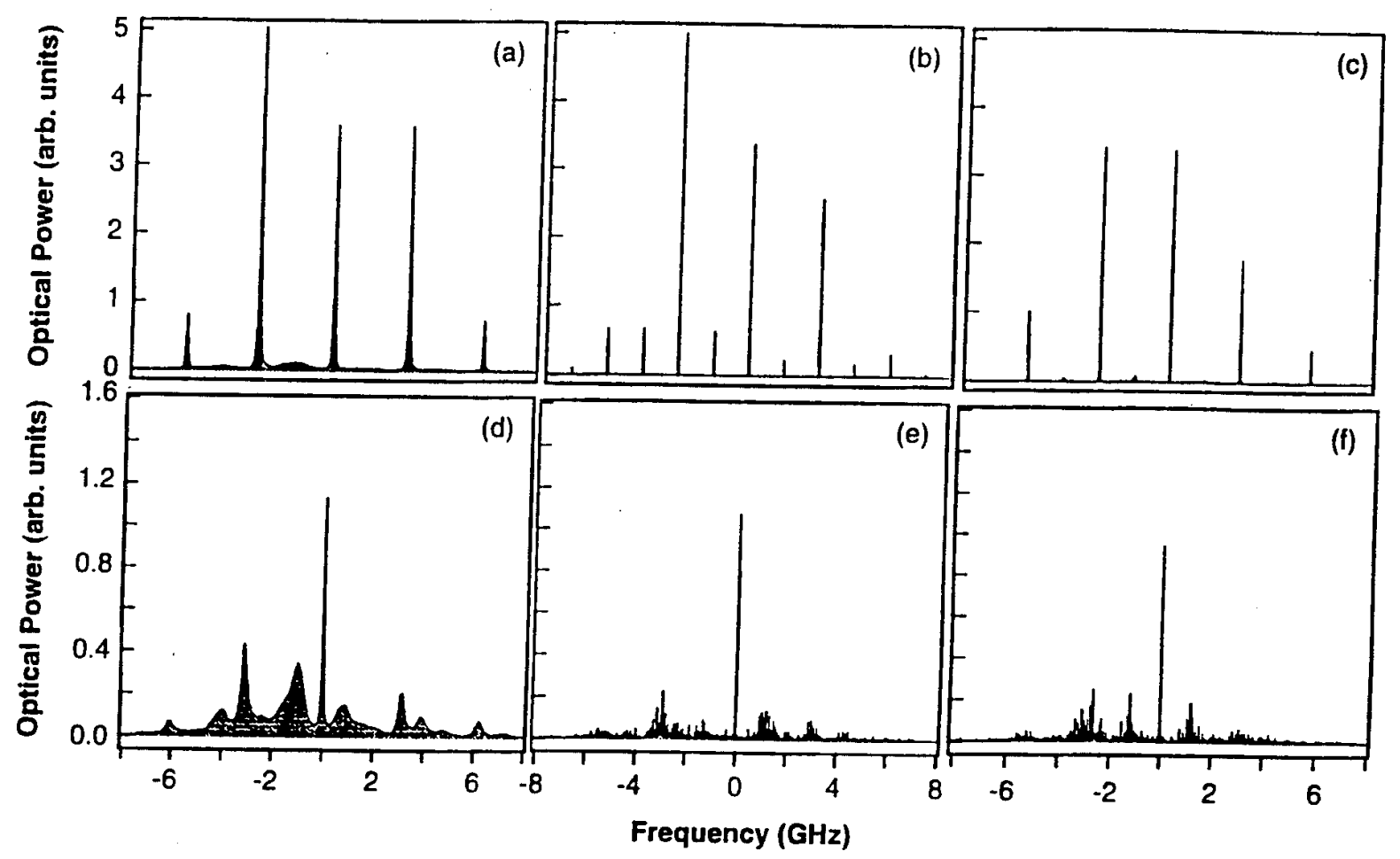

Figure 20. Optical spectra of a semiconductor laser under optical injection at two levels in a period-doubling route to chaos (a)-(c): $\xi=1.7 \times 10^{-2}$ and (d)-(f): $\xi=2.6 \times 10^{-2}$. (a) and (d) are experimentally measured spectra, (b) and (e) are numerically calculated spectra with no noise sources present, and (c) and (f) are calculated spectra with noise sources present. The parameters used in the calculations were experimentally determined using the four-wave mixing technique. Shading under the curves is a visual aid only.

The period-doubling features are most evident in the deterministic spectra, Figure 20(b). A principal difference between the two sets of calculated spectra is that the noise term leads to a broadening of the period-doubling features in Figure 20(c), consistent with the experimental data of Figure 20(a). The noise would tend to obscure the identification of these peaks without our detailed work to determine the dynamic and noise parameters of the laser diode. Broadening of the relaxation oscillation side peaks is accompanied by a reduction in the height of the peaks. Fully developed chaos is observed in the spectrum shown in Figure 20(e), where the broadening in the calculated optical spectra is achieved without any stochastic sources present in Equations 44 to 46 .

Figure 21 depicts the numerically obtained bifurcation diagram of the values of the extrema of the electric field amplitude versus the injection parameter $\xi$ when the noise source terms are not included. Both the initial bifurcation point and first period-doubling point occur at injection levels consistent with the experimental measurements. Only the initial instability region, first period doubling region, and chaotic region exist over an experimentally distinguishable range of 


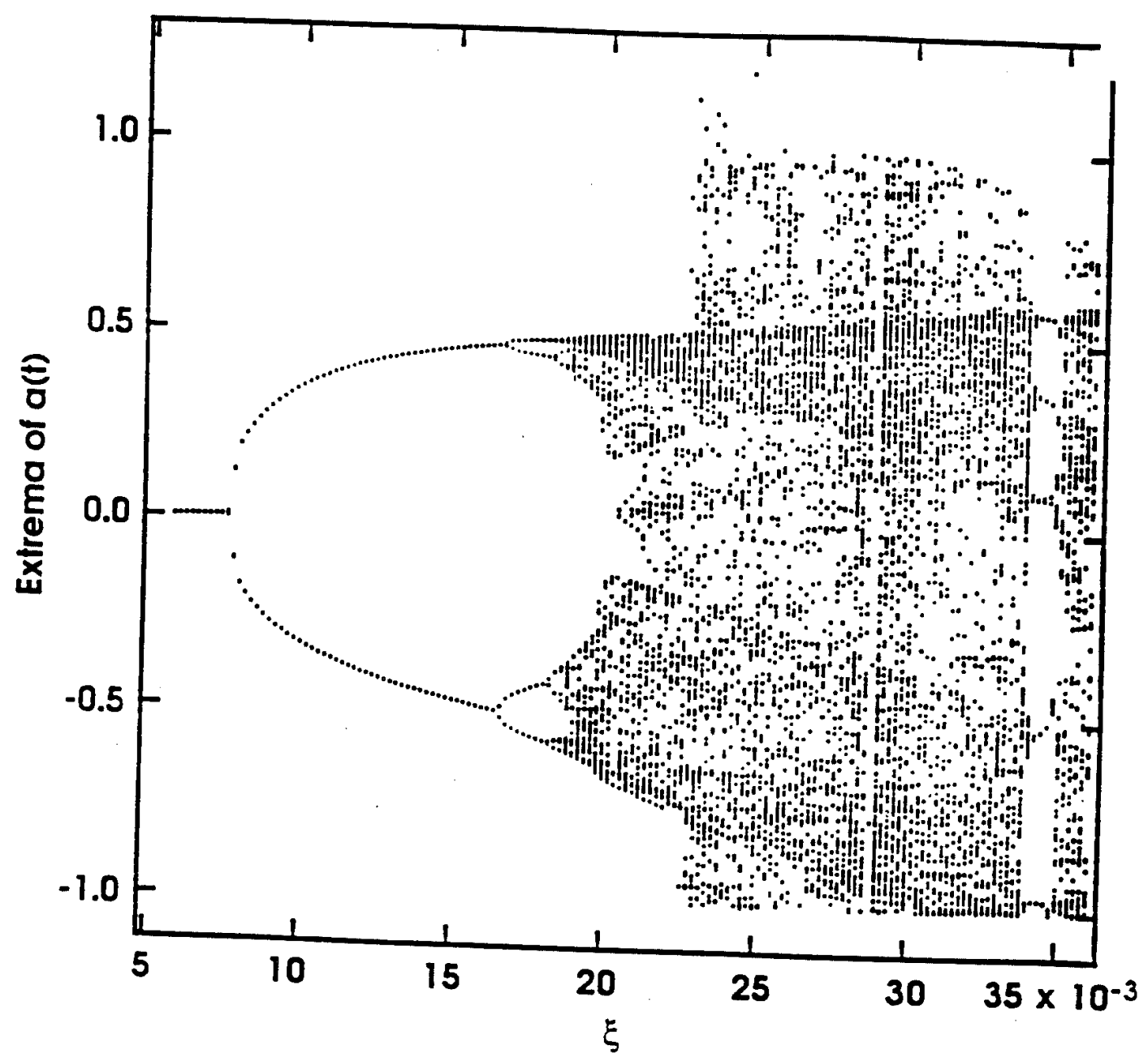

Figure 21. Numerically obtained bifurcation diagram of the extrema of the normalized optical field amplitude, $a(t)$, versus the normalized injection level, $\xi$, showing the laser are used in the calculation.

injection values because of the noise. One may view the effect of spontaneous emission noise as causing a fluctuating optical input. Part of its influence is, effectively, to rapidly move the laser diode output to different injection levels on the bifurcation diagram.

It was also found that relatively minor changes in the linewidth enhancement factor can induce large changes in the calculated bifurcation diagram and optical spectra. The numerical results discussed above were obtained with a linewidth enhancement factor of $b=3.47$, which is well within the range of values $3 \pm 1$ determined by the four-wave mixing experiment [Ref. 21]. We have found that the laser would not develop into a chaotic state if $b \leqslant 3.1$. The transition to chaos can be used to narrow the uncertainty in $b$ to $3.6 \pm 0.4$. 


\subsection{PERIOD-DOUBLING CASCADES AND CHAOS}

In this section, experimental measurements and model calculations based on a single-mode model are combined to give a consistent picture of a laser diode exhibiting a wide range of nonlinear dynamical behavior. We show that a laser diode under optical injection at its freerunning frequency exhibits a region of chaotic dynamics that is bounded as a function of the injection level, and that the laser diode follows a period-doubling route to chaos as the injection level is varied from both above and below the chaotic region. Spontaneous emission into the oscillating mode acts like an additional, fluctuating optical input which broadens and obscures features of the period-doubling cascade. At high injection levels, a new, distinct period doubling occurs. Further, we show that the resonance frequency induced by the coupling between the gain medium and the oscillating field is strongly modified by the injected field. The reverse bifurcation process from chaotic to periodic nonlinear dynamics with increasing optical injection and the existence of a second, distinct period-doubling region have not, to the best of our knowledge, been previously reported in laser diodes. Finally, the observed dynamics are related to models of other systems exhibiting nonlinear dynamics.

We are specifically interested in the situation when the master laser is tuned to the frequency of the free-running slave laser, $\Omega=0$. In this case there is no new modulation frequency imposed on the coupled equations by the external source. The external field does couple the phase into the amplitude equation, providing the third degree of freedom required for chaotic dynamics. A linear stability analysis provides information about the resonant coupling between the carriers and oscillating field. In Figure 22, the nonzero eigenfrequency of the coupled equations is shown as a function of the injection parameter. The linear stability analysis predicts a bounded region of unstable dynamics and a monotonically increasing resonance frequency as the injection level is increased.

To understand the deterministic dynamics and gain global information about the system, the full nonlinear coupled equations are first solved with the noise source terms set to zero. Figure 23 depicts the numerically obtained bifurcation diagram of the values of the extrema of the electric field amplitude, $a(t)$, versus the injection parameter $\xi$. As the injection level is increased, the steady state is destabilized and the relaxation frequency is undamped (Hopf bifurcation), in agreement with the linear stability analysis. Further increasing the injection leads to a perioddoubling bifurcation route to chaos and then a similar, but reversed, bifurcation route out of the chaotic region. To confirm the chaotic nature, the Lyapounov exponents, and the corresponding Kaplan-Yorke dimension of the attractor have been calculated [Ref 42]. For $\xi=0.03$, the Lyapounov exponents are $\lambda_{1}=0.01 \gamma_{c}, \lambda_{2}=0.0$, and $\lambda_{3}=-0.01 \gamma_{c}$ and the Kaplan-Yorke 


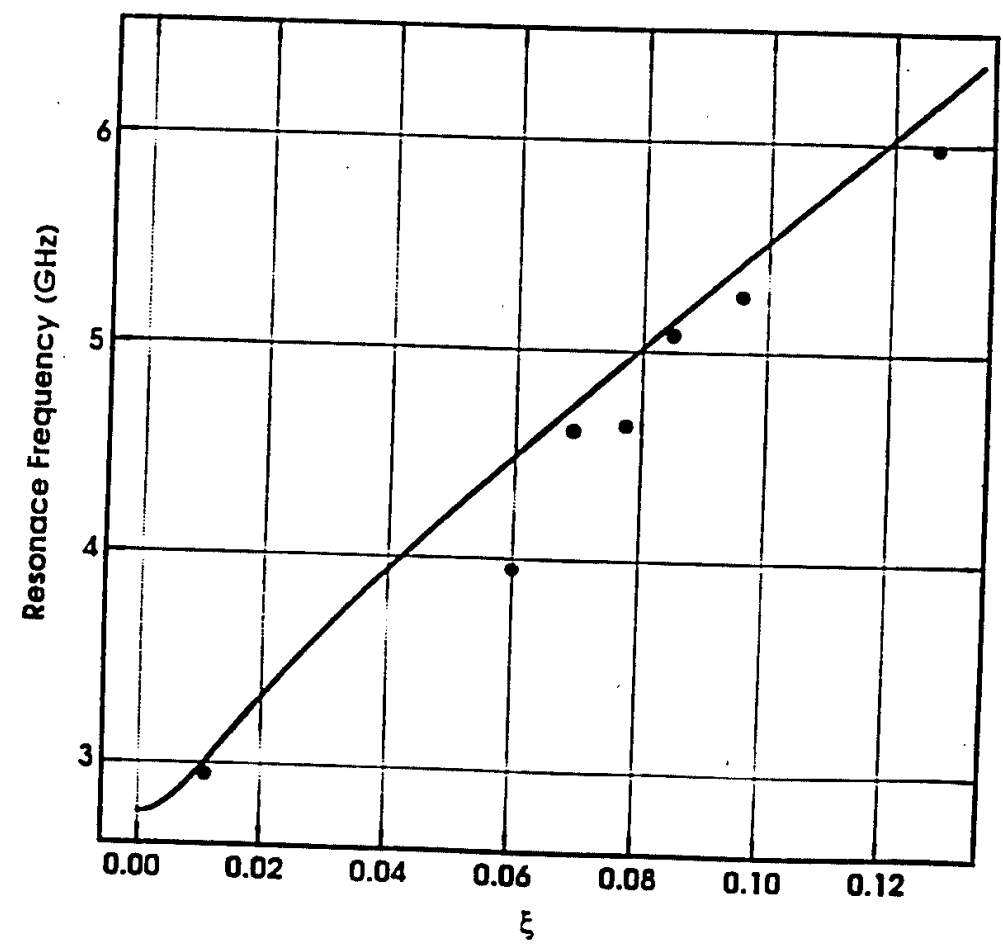

Figure 22. Variation of the resonance frequency as a function of the injection level $\xi$. The colid curve is the calculation from the linear stability analysis and the solid determined parameters for the slave laser operating at the used experimentally as the data.

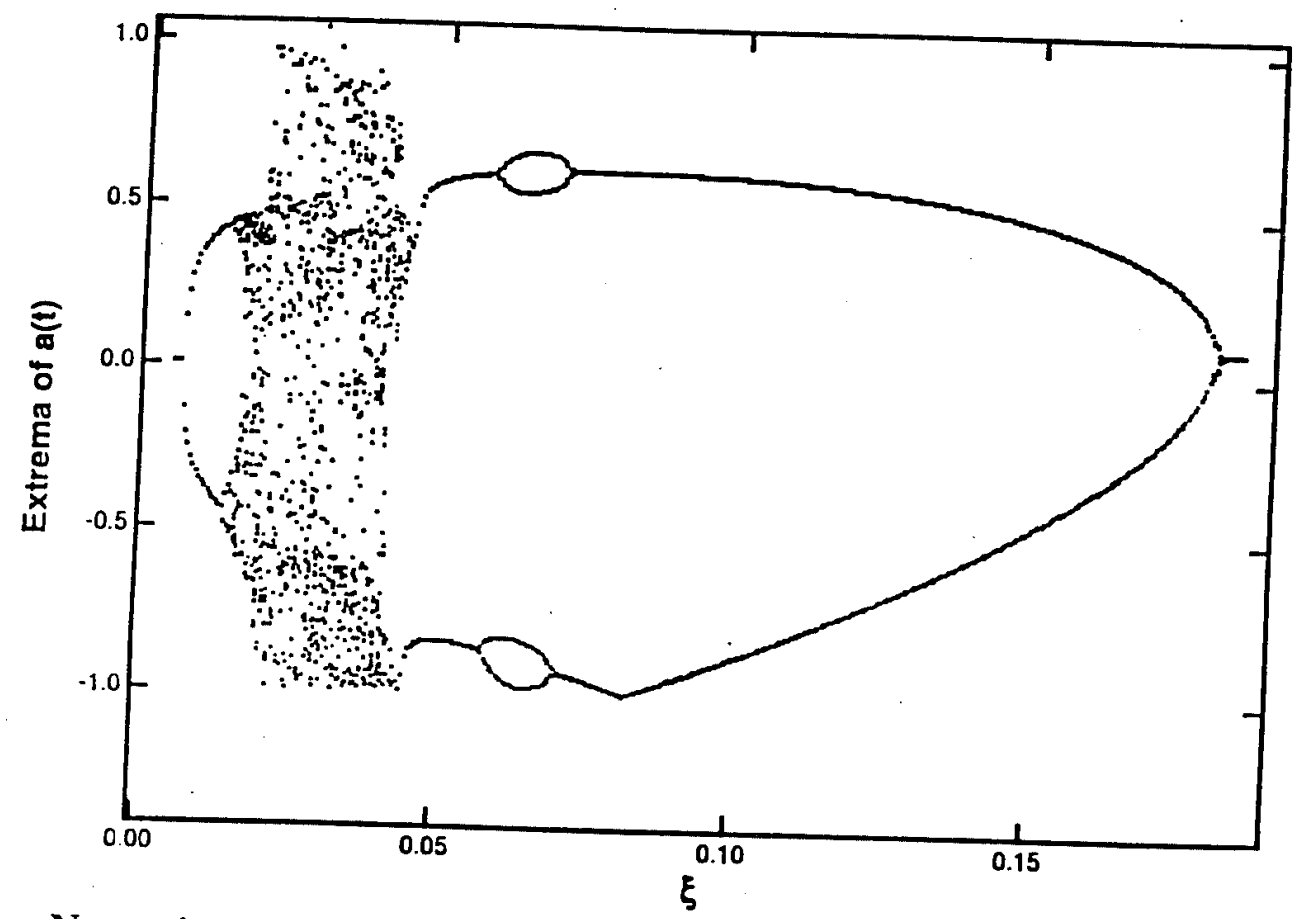

Figure 23. Numerically calculated bifurcation diagram of the extrema of the normalized optical field amplitude, $a(t)$, versus the normalized injection level, $\xi$. Injection
is at the free-running frequency of the slave laser. Experimentally determined
parameters of the semiconductor laser ara unation parameters of the semiconductor laser are used in the calculation. The value
of the linewidth enhancement factor is $b=4$. 
dimension is $\sim 2.5$. The positive Lyapounov exponent is a clear measure that the system is chaotic in this parameter range. At even higher injection levels, the bifurcation diagram shows that the laser undergoes a second period doubling. Finally, at the highest injection levels, the laser diode restabilizes, consistent with the linear stability analysis.

Because the model parameters have been derived from a laser diode carefully characterized using the four-wave mixing technique, we can make a direct, quantitative comparison between experimental observation and the theoretical analysis. The model predictions in Figures 22 and 23 correspond to the slave laser operating at an output level of $9 \mathrm{~mW}$, where $\tilde{J}=0.6$ for the laser under investigation. This is also the operating point for the data described next. The dynamic parameters have been previously published [Refs. 21,38 ] with a specific value of $b=4$ used in the calculations for Figures 22 and 23.

We have previously reported how the laser follows the period-doubling route to chaos, consistent with the bifurcation diagram in Figures 23 [Ref. 38]. Figure 24 shows six optical spectra of the principal mode of the slave laser taken with the injection at the free-running frequency. The frequency is relative to the free-running frequency or, equivalently, the injection frequency. In Figure 24(a), $\xi=1.7 \times 10^{-2}$, and the spectrum is dominated by narrow peaks separated by a frequency spacing of $f_{r}=2.9 \mathrm{GHz}$. These are typical features of highly unstable injection locking [Ref. 41]. In addition, broad period-doubling features appear in the spectrum between the narrow oscillation peaks [Ref. 38]. They are stronger at negative detunings, a reflection of the positive value of $b$. In Figure $24(\mathrm{~b}), \xi=2.6 \times 10^{-2}$, the spectrum becomes dominated by a broad pedestal and many secondary peaks develop, all the energy is shared by them. At this stage chaos has fully developed [Ref. 38] and continues through $\xi=5 \times 10^{-2}$, Figure 24(c). Within the region of chaotic dynamics, a fraction of the oscillating power (up to 35 percent) is shifted from the principal oscillating mode into several of the weak side modes. Over a narrow injection range, the broadened spectrum collapses again into an equally spaced set of narrow features, as shown in Figure 24(d) where $\xi=6 \times 10^{-2}$, and the principal mode regains its full power. Now, however, the separation of the peaks is of the order of $4 \mathrm{GHz}$ and the spectrum is much more strongly shifted to the negative components. At still higher injection levels, Figure $24(\mathrm{e})$ where $\xi=8.5 \times 10^{-2}$, a new, clear period doubling is observed with a further increase of the resonance frequency and relative strengthening of the negative frequency components. The strongest spectral feature is clearly frequency-shifted, frequency pushing induced by the resonant injection. The period doubling peaks and the frequency shifts then steadily decrease in magnitude up to $\xi=0.13$, the largest value of injection that we were able to measure, Figure 24(f). Just above this level the slave laser hopped to a new longitudinal mode. 


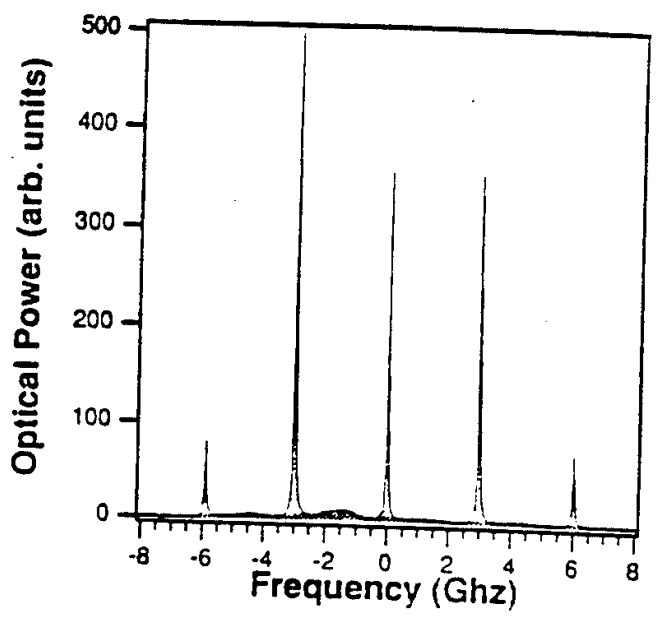

(a) $\xi=1.7 \times 10^{-2}$

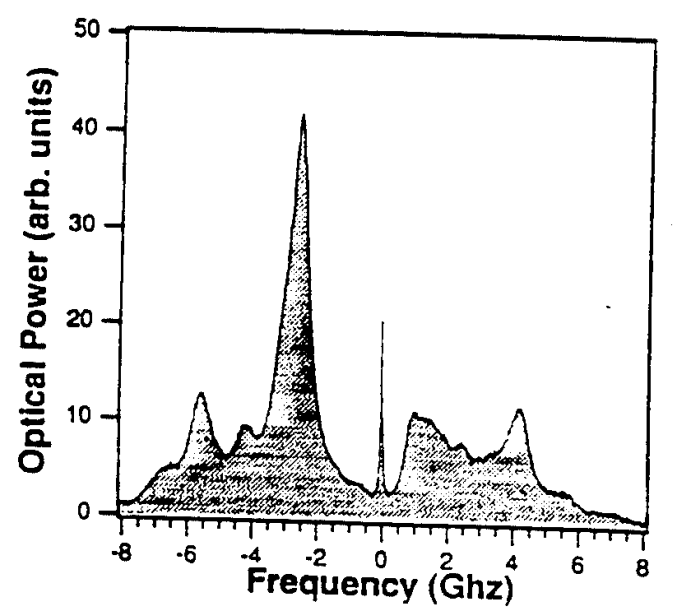

(c) $\xi=5 \times 10^{-2}$

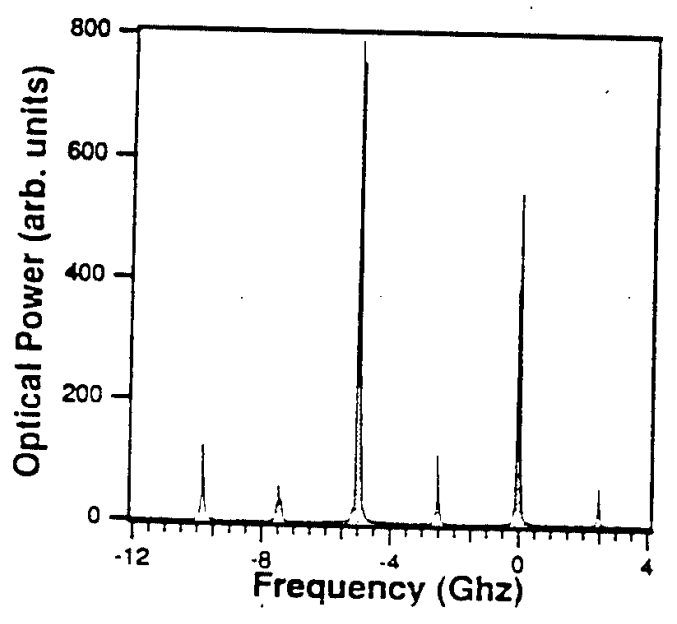

(e) $\xi=8.5 \times 10^{-2}$

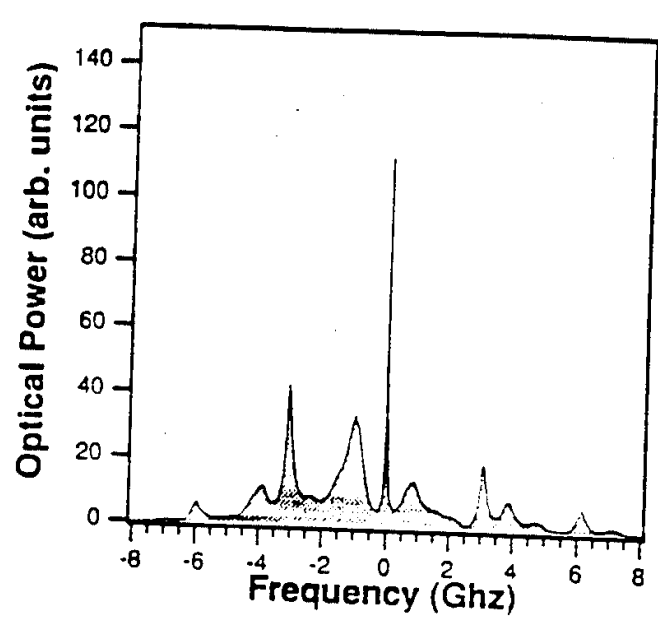

(b) $\xi=2.6 \times 10^{-2}$

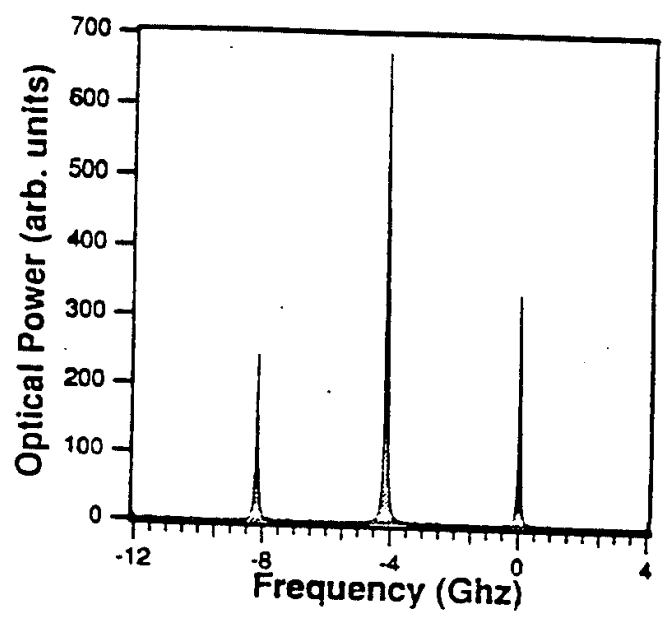

(d) $\xi=6 \times 10^{-2}$

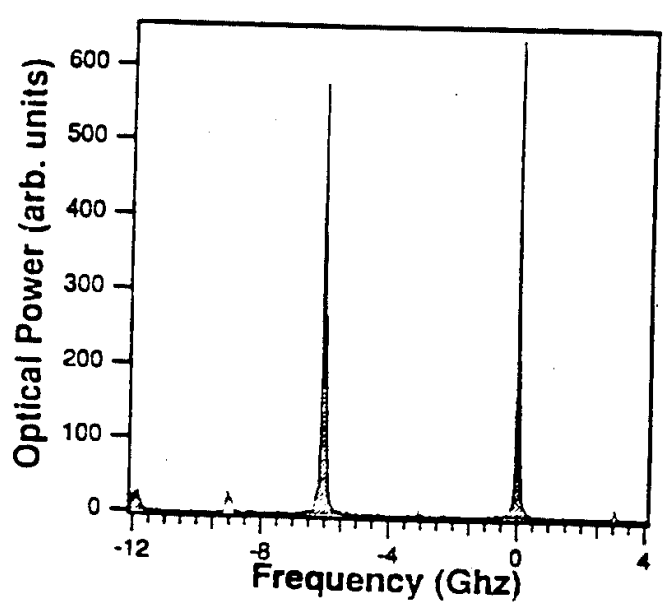

(f) $\xi=0.13$

Figure 24. Measured power optical spectra of the quantum well laser under optical injection at six levels of injection power. (Shading under the curves is a 
Comparison with the calculated bifurcation diagram in Figure 23 shows that the measured spectra are reproduced in the model, though there is discrepancy between the injection levels where the different types of spectra are calculated and observed. Further agreement is supplied by a comparison of the observed and calculated resonance frequencies and onset of oscillatory dynamics shown in Figure 22.

Spontaneous emission into the oscillating mode effectively acts as a second, random source term. We have previously shown how the optical noise spectrum of the free-running semiconductor laser is dominated by this noise and that, away from line center, it is due to both regenerative amplification of the spontaneous emission and four-wave mixing between the spontaneous emission and steady-state oscillating field [Ref. 40]. When both coherent injection and spontaneous emission are present, there is, effectively, a time - varying injected field. This leads to a blurring of the two period-doubling cascades. In the forward cascade, only the first perioddoubling is not obscured, and the new frequency components are severely broadened, as seen in Figure 24(b) and described previously [Ref. 38]. The reverse cascade is completely obscured. This effect can be recovered by including spontaneous emission noise sources in the amplitude and phase equations.

A bounded region of chaotic dynamics and a second, distinct region of period-doubled dynamics has not, to the best of our knowledge, been previously reported in a semiconductor laser. This type of dynamics has been studied numerically in cubic maps and in the biharmonically driven Duffing oscillator and is expected to occur in many dynamical systems with at least two control parameters [Refs. 43, 44]. Alternating periodic and chaotic dynamics have been observed in driven nonlinear $\mathrm{R}$-L-diode circuits when the drive frequency is near-resonant to the circuit resonance or one of its subharmonics [Refs. 45, 46, 47]. A region of chaotic dynamics bounded by period-doubling cascades has been observed experimentally in a $\mathrm{CO}_{2}$ laser with an externally modulated loss [Ref. 48], and recently predicted for semiconductor lasers subject to external optical injection [Ref. 49]. In the semiconductor laser subject to external optical injection, the control parameters are the amplitude and frequency of the injected signal. Unlike in the Duffing oscillator, electronic circuits, or the loss-modulated $\mathrm{CO}_{2}$ laser, we introduce no external modulation frequencies in the dynamical Equations 44 through 46 by using an external field as our injection source which oscillates at the same frequency as the free-running slave laser. There is, however, an effective detuning which is associated with the linewidth enhancement factor, $b$. It represents an offset between the peak of the gain and the laser oscillation frequency [Refs. 10, 12]. By appropriately detuning the injection frequency, or by varying the value of $b$, one can observe completely different dynamics, including stable injection locking at all injection levels 
[Refs. 37, 38, 41]. The external injection modifies the key resonance frequencies of the coupled equations. We observe the strong modification of the carrier-field resonance frequency describing the photon-carrier coupling.

\subsection{DISCUSSION}

The excellent qualitative and very good quantitative agreement between our experimental data and the numerical calculations is very strong evidence that the single-mode model coupling the complex oscillating field with the carrier density captures the important physics of a nearly single-mode laser subject to external optical injection, at least up to the point of the observed longitudinal mode hop. The model includes the dependence of the gain on both the carrier density and circulating field intensity. Key rate parameters, and the strength of the spontaneousemission noise, can be determined in the weak injection limit, allowing the model calculations to be based completely on experimentally determined data. By suppressing the noise in the model calculations, we are able to see that when the laser diode is subject to resonant optical injection, it shows dynamics similar to a variety of other systems. However, a novel feature is that these dynamics are induced without introducing any new external modulation frequency in the coupled equations. This point emphasizes the critical role of the linewidth enhancement factor, $b$, in determining the nonlinear dynamics.

The nonlinear dynamics of a laser diode under external optical injection is sensitive to a variety of laser parameters. Here, we have investigated optical injection at the free-running frequency of the laser diode. Detuning the injection frequency will change the nonlinear dynamics and is predicted to lead to different routes to chaos for lasers which can be modeled by the coupled equations for the complex oscillating field and population inversion [Refs. 12, 34, 50]. In the analysis, the effect of side modes was not included. The single-mode model used here cannot, of course, account for the partition of power to the side modes and further work is needed to quantify the effect of the side modes. The good agreement between the spectra calculated from the single-mode model with noise and the experimental data indicates that the high frequency dynamics of the system are not dominated by the presence of the side modes for these experimental conditions.

There is still some uncertainty in the value of the linewidth-enhancement factor, $b$, though the data from the nonlinear dynamics regime reduces this to the same relative uncertainty, \pm 10 percent, as the other parameters that were determined in the perturbation limit. We achieved our best quantitative agreement between measurements and modeling of the perioddoubling route to chaos from low injection levels using $b=3.5$. However, we did not observe 
the separate period-doubling bubble until $b=4$. The larger value of $b$ increased the range of injection where chaos was observed, in better agreement with the data, but led to an onset of chaos at lower injection levels than were observed experimentally. Further, even with the higher $b$ value, the separate period-doubling bubble did not cover the range of injection levels observed experimentally. We did not perform a full check of the spectra over the possible range of uncertainty of all of the parameters, including the uncertainty in the spontaneous-emission noise level, of the model. We do not believe that the experimental accuracy of the data warrants further tweaking of the parameters. Further, it is important to remember that we retain the perturbation model of the gain. The full nonlinear dynamical model still incorporates a gain parameter, $g(N, S)$, that is a linear function of the carrier density modulations and the photon density modulations with no cross term. This approximation may not be fully valid in the strongly nonlinear regime. However, it is significant that the perturbation model can be used so successfully. More complicated models involve the introduction of extra assumptions and extra fitting parameters. While they would certainly be able to fit the data, the data probably could not sufficiently distinguish between different models. Therefore, the use of more complicated models is not warranted until there are more dramatic differences between the data and the model predictions. 


\subsection{DETUNED INJECTION IN THE STRONG SIGNAL REGIME}

When the injection frequency is detuned from the free-running frequency in the strong injection regime, a complicated mapping of laser dynamics is observed. Figure 25 shows the types of behavior observed in the high injection regime. The dynamics observed at zero detuning represent a cut in this two-dimensional mapping. For large regions of positive detuning, a sizable fraction of the output power is shifted to other longitudinal modes. Complete mode hops to other longitudinal modes can be induced. This behavior is beyond the scope of the singlemode model. The dynamics represented by the optical spectra in the different regions of the mapping can be qualitatively reproduced from the model equations.

The region of stable injection locking is of interest because wan again attempt to quantitatively recover the observed dynamics using a perturbation analysis and use the observed noise spectra as a test of the linearized model. For injection levels outside of the weak-injection limit, the stable, injection-locked oscillating point is induced when the master laser is shifted in frequency by $\Omega$, relative to the free-running frequency. The phase is shifted by $\phi_{1}$, relative to the injection signal where:

$$
-\Omega=\eta a_{i}\left(b \cos \phi_{1}-\sin \phi_{1}\right)
$$

The amplitude is shifted by $A_{0 a_{l}}$ and the carrier density by $N_{0} \tilde{n}_{l}$, where

$$
\begin{aligned}
& \tilde{n}_{1}=-\frac{2 \tilde{J} a_{I}\left(1-\gamma_{p} / \gamma_{c}\right)}{1+\gamma_{n} / \gamma_{s}} \\
& -\frac{\eta}{\gamma_{c}} a_{i} \cos \phi_{1}=\frac{\gamma_{n}}{2 \gamma_{s} \tilde{J}} \tilde{n}_{1}-\frac{\gamma_{p}}{\gamma_{c}} a_{1} .
\end{aligned}
$$

These equations are approximately valid for $a_{i}<0.1$ and $\left(\gamma_{n} \tilde{n}_{l} / \gamma_{S} \tilde{J}\right)<0.1$.

Using this steady-state operating point, the new linearized equations can be written as

$$
\frac{d a}{d t}=\frac{\gamma_{c} \gamma_{n}}{2 \gamma_{s} \tilde{J}} \tilde{n}-\left(U+\gamma_{p}\right) a-V \tilde{\phi}+F_{\text {real }} /\left|A_{O}\right|
$$




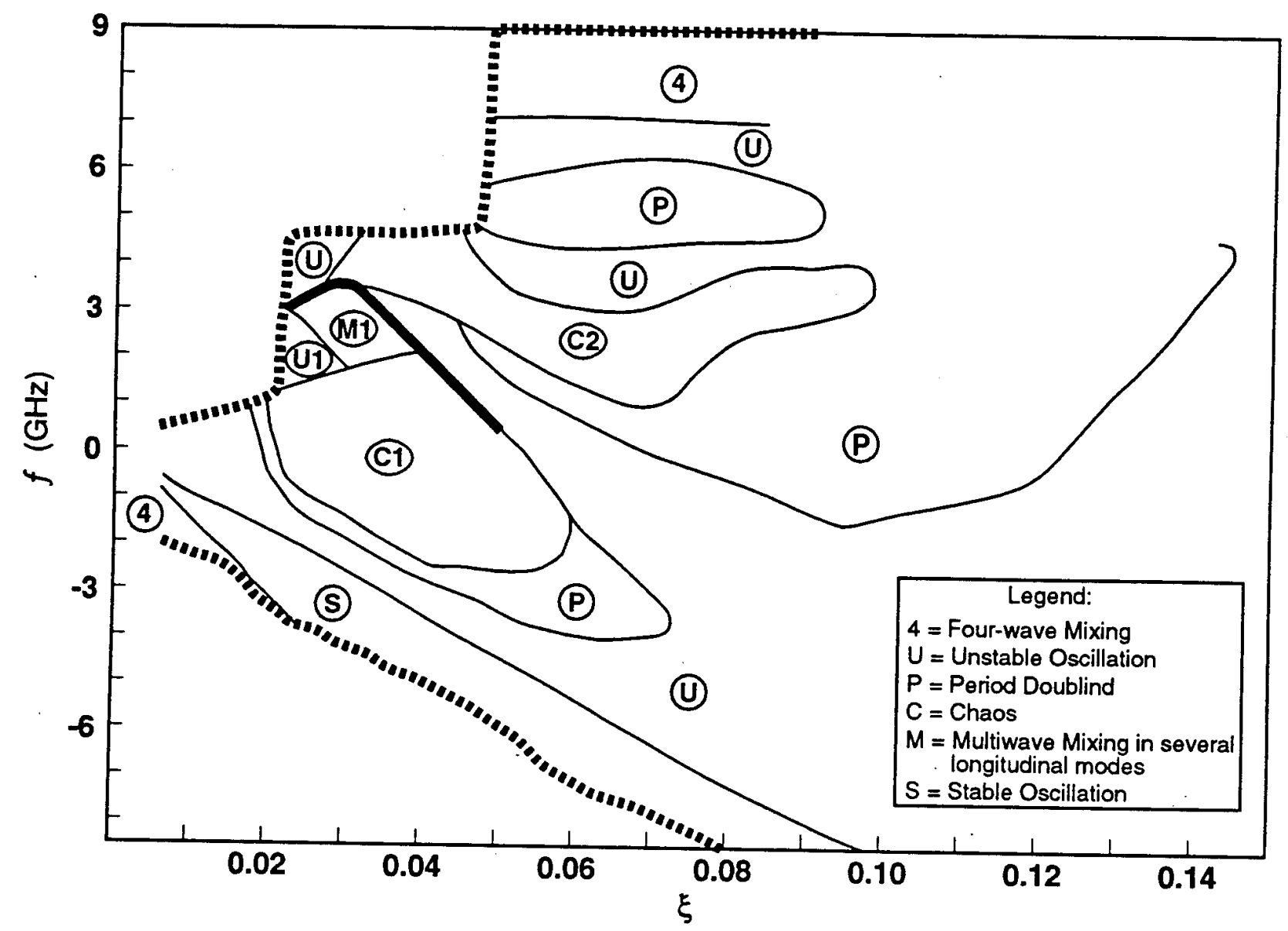

Figure 25. A mapping of the regions of different laser dynamics as a function of injection level and detuning. The thick solid lines represent abrupt mode hops with the two outermost lines being mode hops away from the principal mode, while the interior line is an abrupt transition back to a predominantly single-mode operation in the principal mode as the detuning is increased. Other boundaries are smooth. Large regions of positive detuning and the $C 1$ region have large fractions of the output power in modes other than the principal mode.

$$
\begin{aligned}
& \frac{d \tilde{\phi}}{d t}=\frac{b \gamma_{c} \gamma_{n}}{2 \gamma_{s} \tilde{J}} \tilde{n}-\left(V+b \gamma_{p}\right) a-U \tilde{\phi}-F_{\text {imag }} /\left|A_{O}\right| \\
& \frac{d \tilde{n}}{d t}=-\gamma_{s} \tilde{n}-\gamma_{n} \tilde{n}-2 \gamma_{s} \tilde{J}\left(1-\gamma_{p} / \gamma_{c}\right) a+\gamma_{s} F_{c} .
\end{aligned}
$$

Here, the noise terms induce fluctuations about the new injection-locked steady-state oscillating point, $U=\left(\eta / \gamma_{c}\right) a_{i} \cos \phi_{I}$ and $V=\left(\eta / \gamma_{c}\right) a_{i} \sin \phi_{I}$. When Fourier transformed, these equations generally yield complicated solutions. However, a particularly simple solution is found for 
the case where $\phi_{1}=0$. In this case, the previous linear formulas, Equations 38 through 41 , for the spectra carry over to the strong injection-locked regime except for two substitutions: $\gamma_{r} \Rightarrow \gamma_{r}+U$ and $\Omega_{r}^{2} \Rightarrow \Omega_{r}^{2}+\left(\gamma_{s}+\gamma_{n}\right) U$

The model makes interesting, and potentially useful, predictions about the injection-modified modulation characteristics. Figure 26 shows the predicted changes in the amplitude noise spectra, assuming strictly single-mode operation and only spontaneous emission into the lasing mode as a noise source. The free-running spectrum is shown with the spectra at two injection levels. The relaxation resonance becomes broadened and eventually disappears as the diode changes from underdamped to overdamped oscillations under the influence of the external field. It is important to note that the optical frequency of the injection laser must shift with increasing injection level to maintain the $\tilde{\phi}=0$ operating point. Also, the noise power decreases considerably, though the regenerative amplification and four-wave mixing signals from an external source would be equally reduced. Overall, however, under stable injection locking in the strong injection regime, the modulation characteristics are markedly improved. The wider modulation bandwidth is achieved with a constant amplitude injection signal which can be from a laser with very poor high-frequency performance characteristics.

Experimentally, we have observed more than an order of magnitude decrease in the amplitude of the noise spectrum, relative to the free-running case. This is most clearly shown in the data from the amplitude spectra. Figure 27 shows representative amplitude spectra in the vicinity of the relaxation-resonance peak. They show the broadening of the resonance peaks and the decrease in signal levels predicted by the model. The changes, however, do not quantitatively match the predictions. At injection levels $\xi \geq 0.1$, the quantitative differences can be explained by the fact that our master laser is not a purely monochromatic sine wave but has a noise spectrum of its own. At high injection levels, this noise source dominates the internal spontaneous emission noise and influences the observed amplitude spectra. At lower injection levels, there is still a lack of quantitative agreement which cannot be explained by the master laser's noise spectrum. Work is currently in progress to ascertain whether the observed noise level is consistent with the full nonlinear equations.

The low frequency amplitude spectrum is influenced by the weak side modes. Under stable injection locking in the strong injection regime, the power in the side modes can be severely reduced [Refs. 28, 29]; we observe this in the principal mode amplitude spectra at the lower injection levels. At higher levels, the noise in the master laser again influences the spectra and we do not see the side modes depleted to the extent observed by others [Refs. 28, 29]. When the 


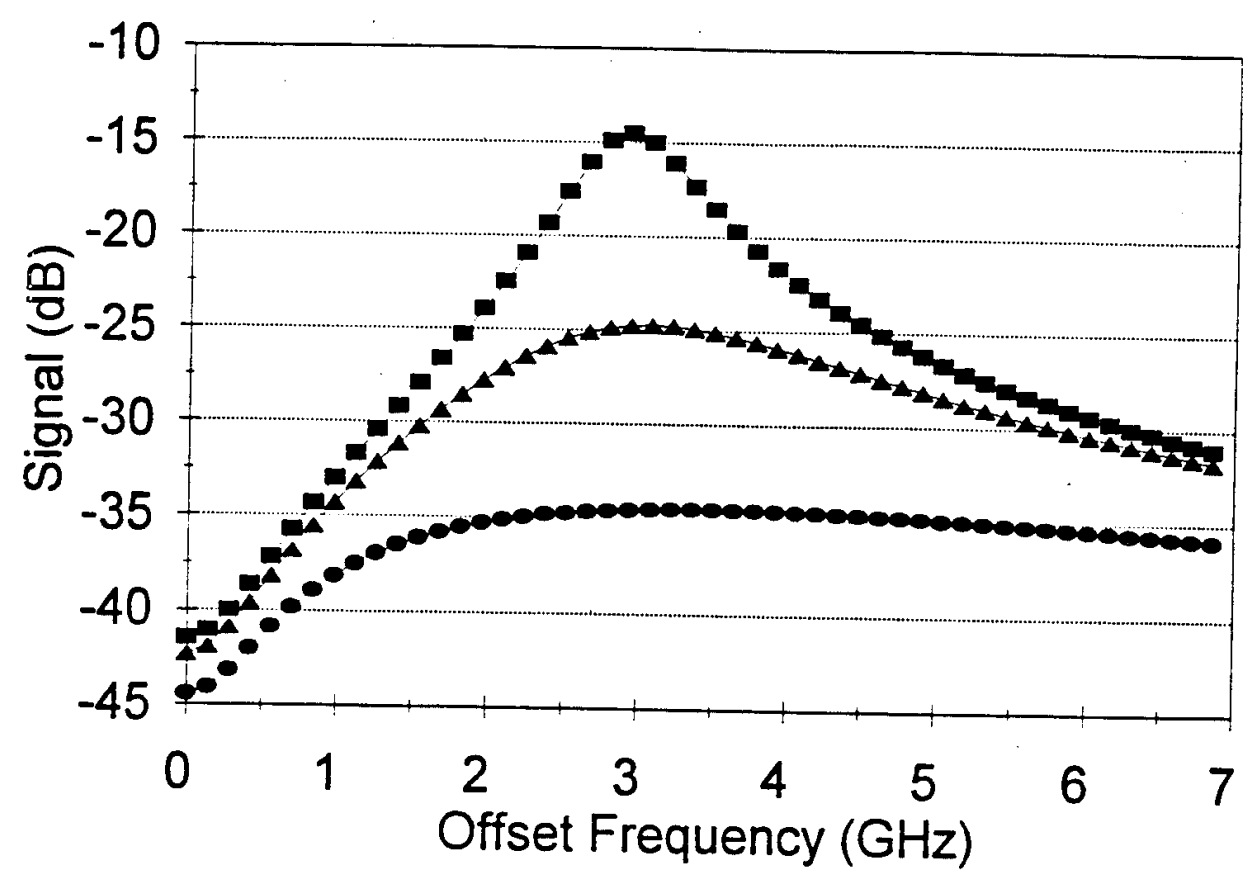

Figure 26. Calculated changes in the amplitude noise spectra of a single-mode laser, where the dominant noise source is spontaneous emission, due to stable injection locking by an external laser whose frequency is tuned so that $\tilde{\phi}=0$. The three different injection levels are: squares $-\xi=0$, triangles $-\xi=0.06$, ovals $-\xi=0.2$. (Calculations use the parameters for the quantum-well laser diode operating at $9 \mathrm{~mW}$.)

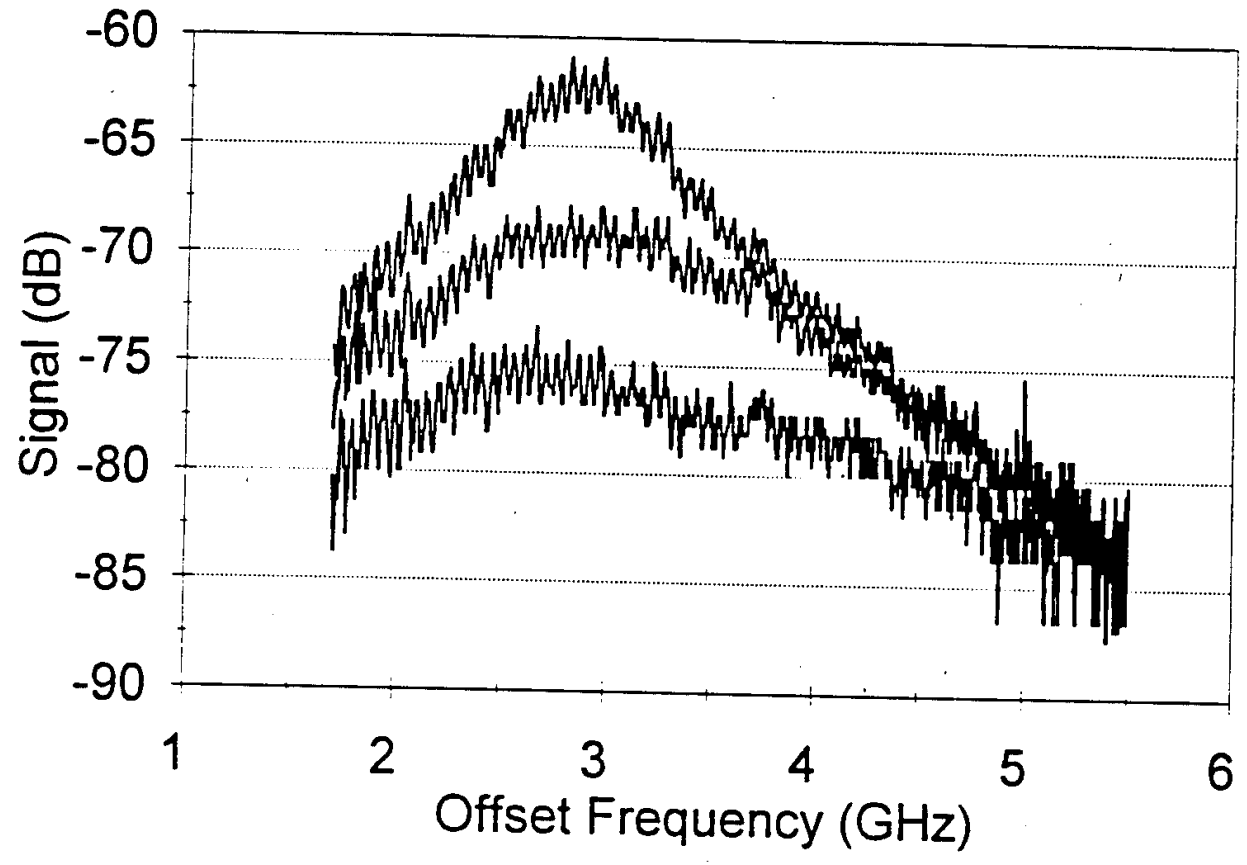

Figure 27. Measured changes in the amplitude noise spectra in the vicinity of the freerunning relaxation-resonance peak due to stable injection locking by an external laser whose frequency is tuned so that the amplitude of the noise spectra is minimized. The three different injection levels are: top $-\xi=0$, middle $-\xi=0.06$, and bottom $-\xi=0.2$. 
total output is monitored, we see some depletion in the lower frequency noise spectra, but not to the extent that the principal mode noise is depleted. This is further corroboration that the excess noise, relative to the spontaneous emission level, is due to side modes that are not perfectly correlated with the principal mode. If the excess noise were due to carrier shot noise, its level would not be reduced by the external injection to the extent that we observe. Imperfect correlation between the principal mode and weak side modes, due to inhomogeneous gain characteristics or mode dependent losses, would yield the noise spectra we observe. As with the other spectra, the total output spectra show increased noise at high injection levels due to the noise characteristics of the master laser. Further work is underway to quantify these characteristics. 


\subsection{VERTICAL-CAVITY SURFACE-EMITTING LASERS}

Recently, a new type of laser structure has become the subject of considerable research interest, the VCSEL. These lasers have a number of interesting properties relative to the conventional edge-emitting laser diodes including surface-normal emission, a circular output beam, and ready fabrication into two-dimensional arrays [Ref. 51]. In addition, these lasers can be fabricated with dimensions approaching the wavelength of light emitted by the cavity, allowing novel physics to be observed [Ref. 52]. We have initiated an investigation of VCSELs to see how well our modeling and optical injection techniques work in the parametrization of these lasers. Here, we are able to report only preliminary results on the spectral and polarization characteristics of these novel structures.

The VCSEL under study is similar to devices previously described in the literature [Refs. 53, 54]. The device consists of GaAs/AlGaAs quantum wells embedded within a wavelength-thick spacer region which is sandwiched between two highly reflective distributed Bragg reflectors. The emission wavelength is $\sim 850.1 \mathrm{~nm}$. Due to the short cavity length (but relatively large emitting surface) of these devices, the laser supported only one longitudinal mode but could support multiple transverse modes [Ref. 53]. Further details are given in [Refs. 53, 54]. A distinct threshold below $4 \mathrm{~mA}$ is observed, as well as kinks in the output characteristic between 4 and $6 \mathrm{~mA}$. At threshold, the output has a distinct linear polarization and a single transverse mode output which appeared to be the TEM 00 mode based on its single lobe spatial characteristic. Above the kink near $6 \mathrm{~mA}$, the laser oscillated in multiple longitudinal modes. Between these two operating points two different modes of observation were observed at different times during the 6-month period of the VCSEL operation. Initially, the laser maintained the single mode operation and polarization direction up to the kink near $6 \mathrm{~mA}$. Later, however, the laser showed different behavior, oscillating in the orthogonal polarization direction with a threshold just above $4 \mathrm{~mA}$. The different operating polarization operating characteristics were observed after the optical alignment had been changed. However, we have not been able to determine the cause of the change in operation.

The orthogonal polarization components are shifted in frequency but appear to have the same spatial characteristics. Figure 28 shows the frequency separation as a function of injection current. The component that oscillated at the higher frequency had the lower threshold but the lower frequency component eventually dominated the output. This is shown in Figure 29. Based on the threshold characteristics, this higher threshold mode never appears in the singlepolarization case. 


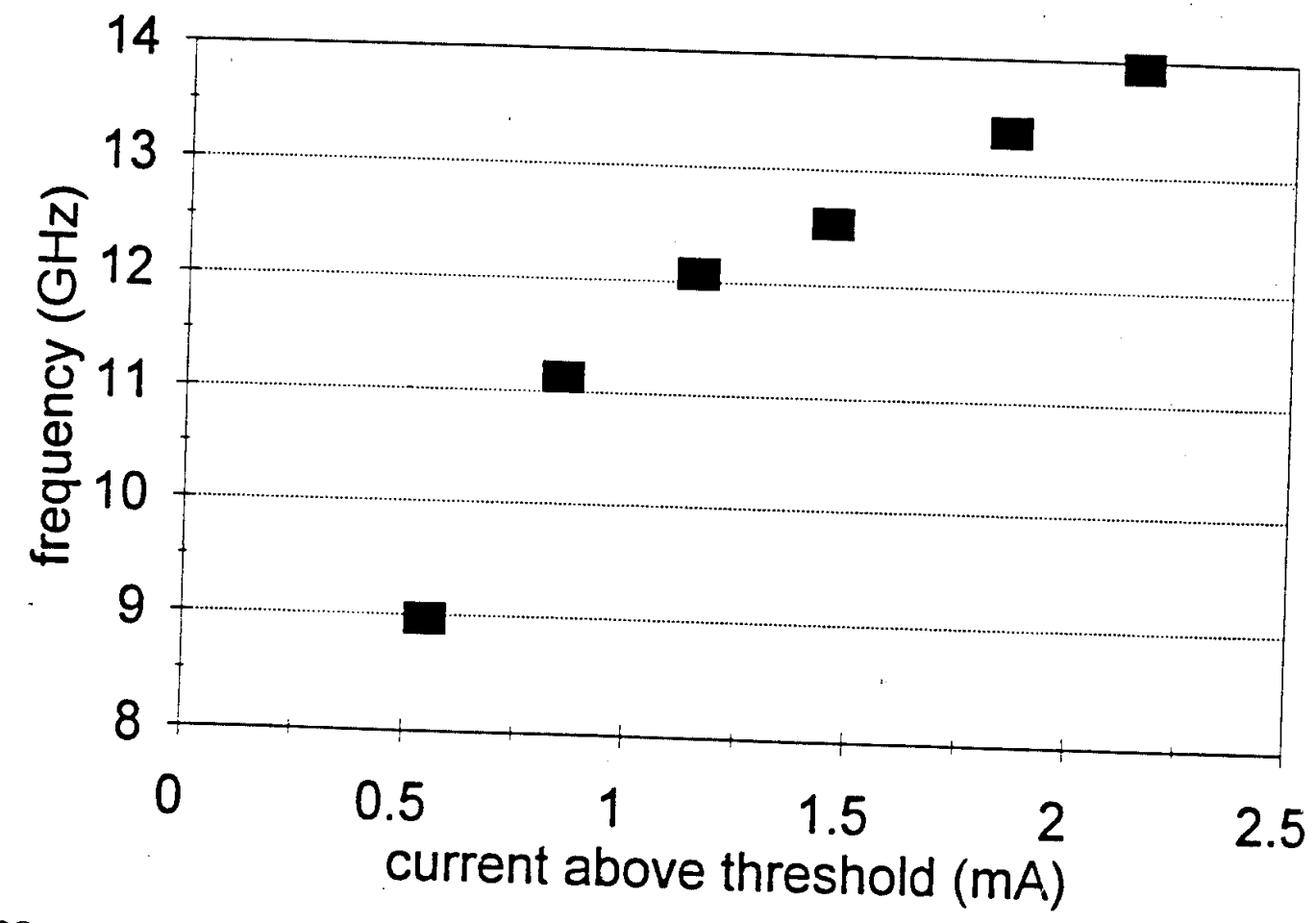

Figure 28. The frequency spacing between the two orthogonally polarized modes as a function of injection current. The higher frequency polarization component
has the lower threshold.

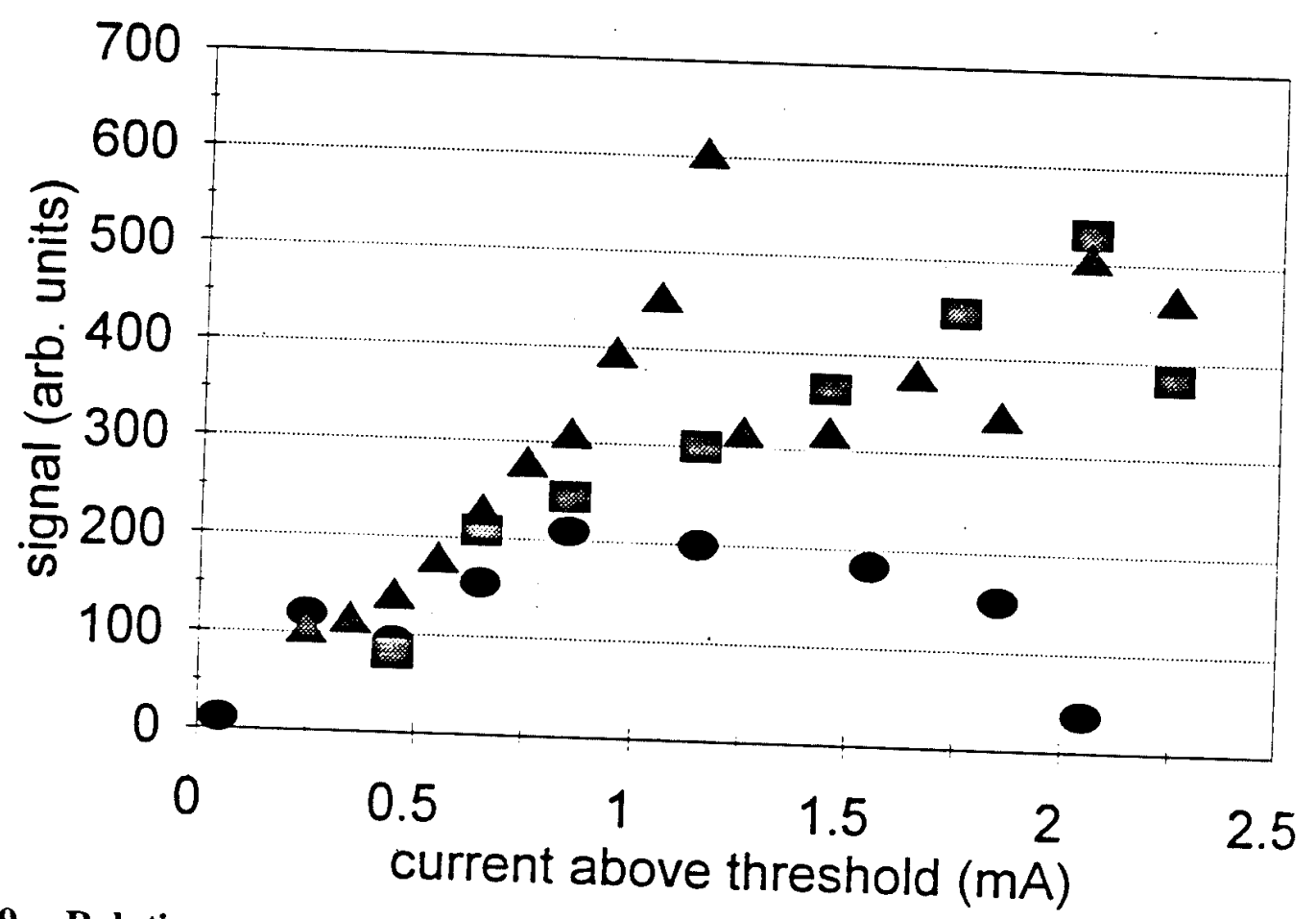

Figure 29. Relative output powers of the two polarization components, and singlepolarization operation, as a function of injection current. The triangles are the squares are the data taken with single-polarization operation. The ovals and correspond to the low threshold and VigEL in dual-polarization operation and 
Figure 30 compares the linewidths of the lowest order spatial modes when measured with the optical spectrum analyzer. Notice the much larger linewidth that accompanies the dual polarization mode of operation. The total output power is similar in the one and two polarization-component cases. The linewidth of the lowest order spatial mode reaches a minimum in the single-polarization, single spatial-mode range of operating currents. However, the linewidth under the single polarization, multitransverse mode operating condition is more than an order of magnitude less than the dual-polarization linewidth.

The microwave power spectrum from the fast photodiode signal for one operating current, $5 \mathrm{~mA}$, is shown in Figure 31. The key point in this figure is that there is a resonance around $370 \mathrm{MHz}$ when only one polarization is monitored, there is essentially no resonance when both polarizations are simultaneously monitored. The residual signal in the lower curve is due to slight transmittance differences between the two polarizations as they propagate through the train of optical components. The two polarizations are exhibiting antiphase dynamics, maintaining a constant overall output power but switching between the two modes. The antiphase switching resonance frequency changes with injection current, as shown in Figure 32. When the laser operated in the single polarization operating condition, there was no resonance below $1 \mathrm{GHz}$. For this operating point, all the microwave spectra exhibited a resonance in the neighborhood of $4.6 \mathrm{GHz}$ that appeared to be the relaxation resonance. The relaxation resonances could be identified by their characteristic dependence on the injection current, as shown in Figure 33. The resonance frequency approximately shows the expected linear relation with the square of the pump parameter. At higher injection currents (when the laser operated under one dominant polarization but in multiple transverse modes) the laser exhibited no resonances except the highfrequency relaxation resonance. The laser exhibits very different dynamics depending on the polarization characteristics. The higher order transverse modes do not exhibit the strong antiphase dynamics but operate simultaneously with the lower order modes

Due to difficulties in achieving an overlap of frequencies between master and slave lasers, and simultaneously detecting sufficient signal, we were unable to quantitatively measure the optical injection spectrum in this laser. Very preliminary measurements supported the identification of the high-frequency resonance in the microwave spectrum with the relaxation resonance. Measurements in the single-polarization, single spatial-mode operating condition show, qualitatively, the kind of regenerative-amplification and four-wave mixing characteristics previously observed. However, we were unable to make quantitative measurements because it was not clear that our injection signals were in the small-signal regime. The injection laser could induce unstable dynamics. No measurements were made of the dual-polarization operating condition because the master laser catastrophically failed during the experiment. 


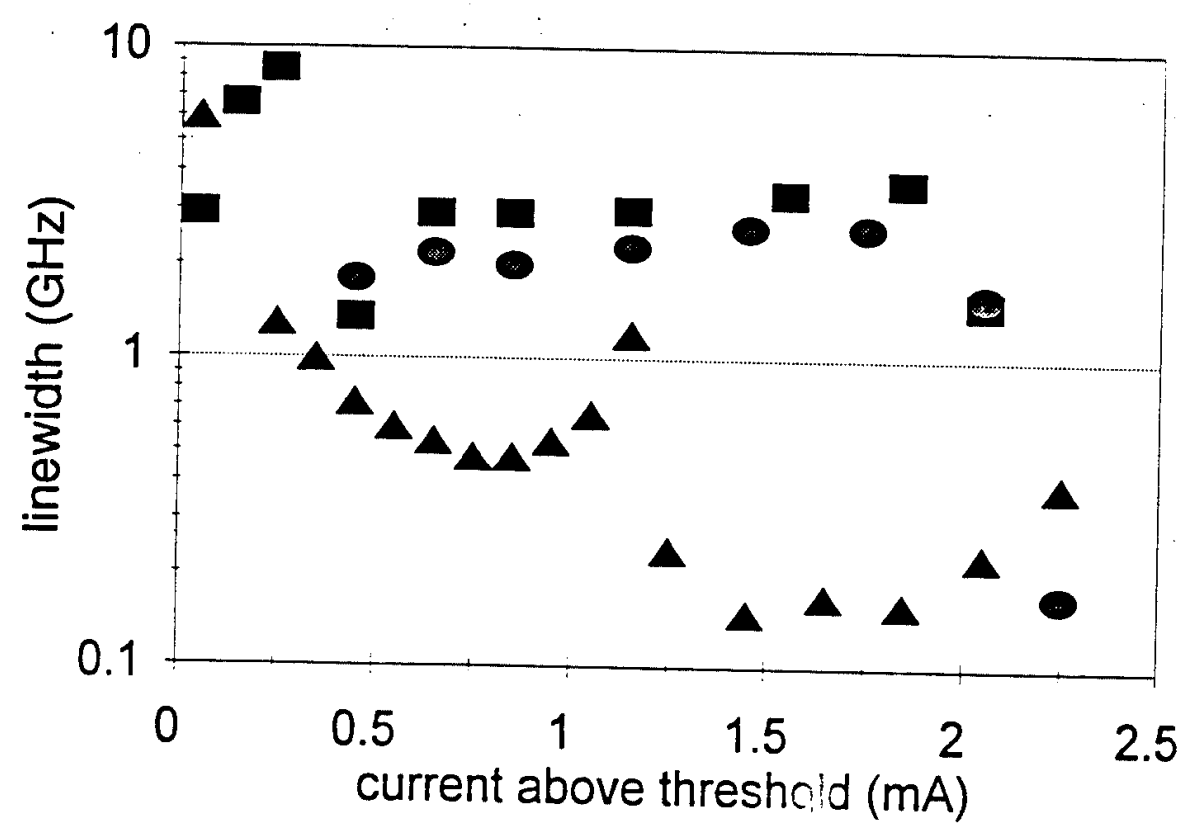

Figure 30. The linewidths of the lowest order spatial modes as a function of injection current. The triangles are the data taken with the VCSEL in singlepolarization operation. The ovals and squares are the data taken with the VCSEL in dual-polarization operation and correspond to the low threshold and high threshold components, respectively. Operation in the twopolarization condition yields significantly larger linewidths than does the single
polarization state.

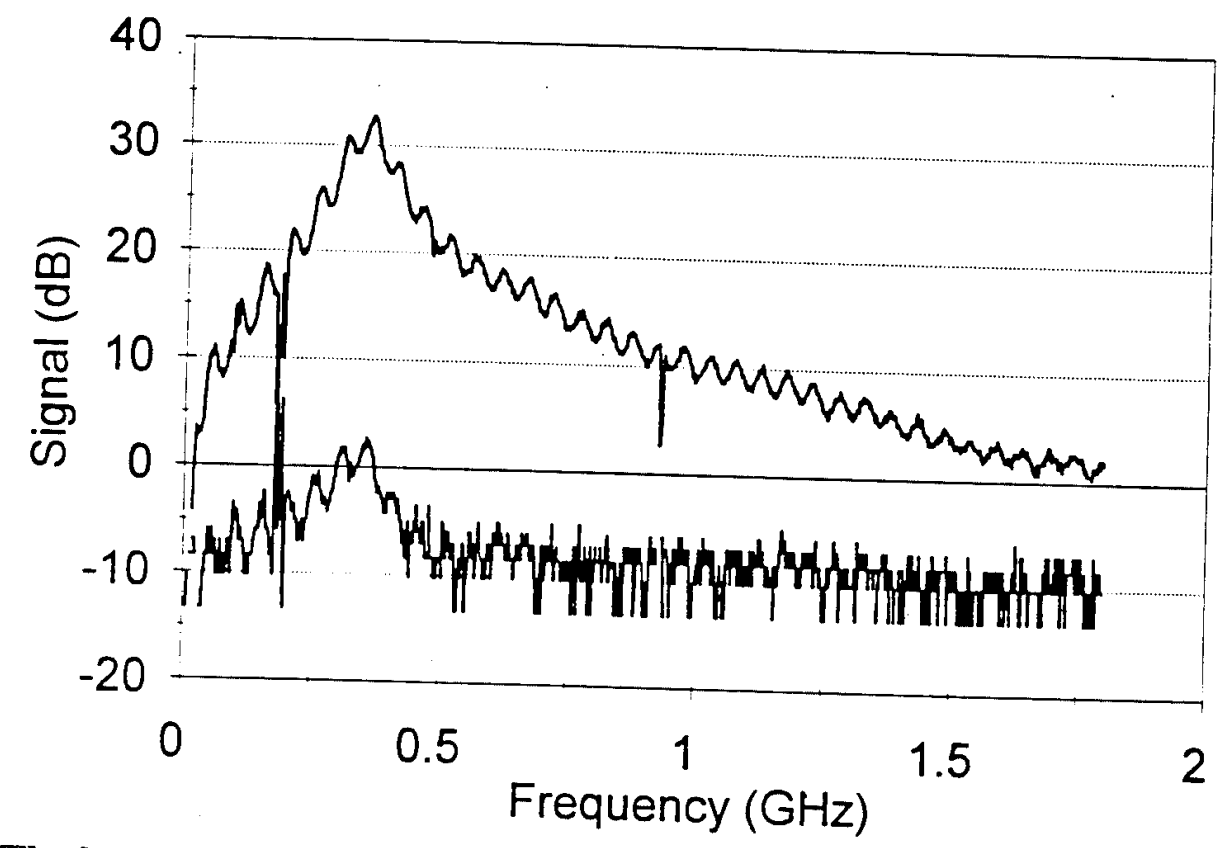

Figure 31. The low frequency microwave spectra of the output of the VCSEL when detected by a fast photodiode. The upper curve shows the spectrum when the polarization passed to the detector is along one of the two orthogonal polarization components. The other component gives an essentially identical curve. The lower curve is the spectrum when the polarization passed to the detector is $45 \mathrm{deg}$. between the two principal axes. The resonance at $370 \mathrm{MHz}$ is not 400 and 900 the single polarization mode is operating. The sharp spikes at artifacts. 


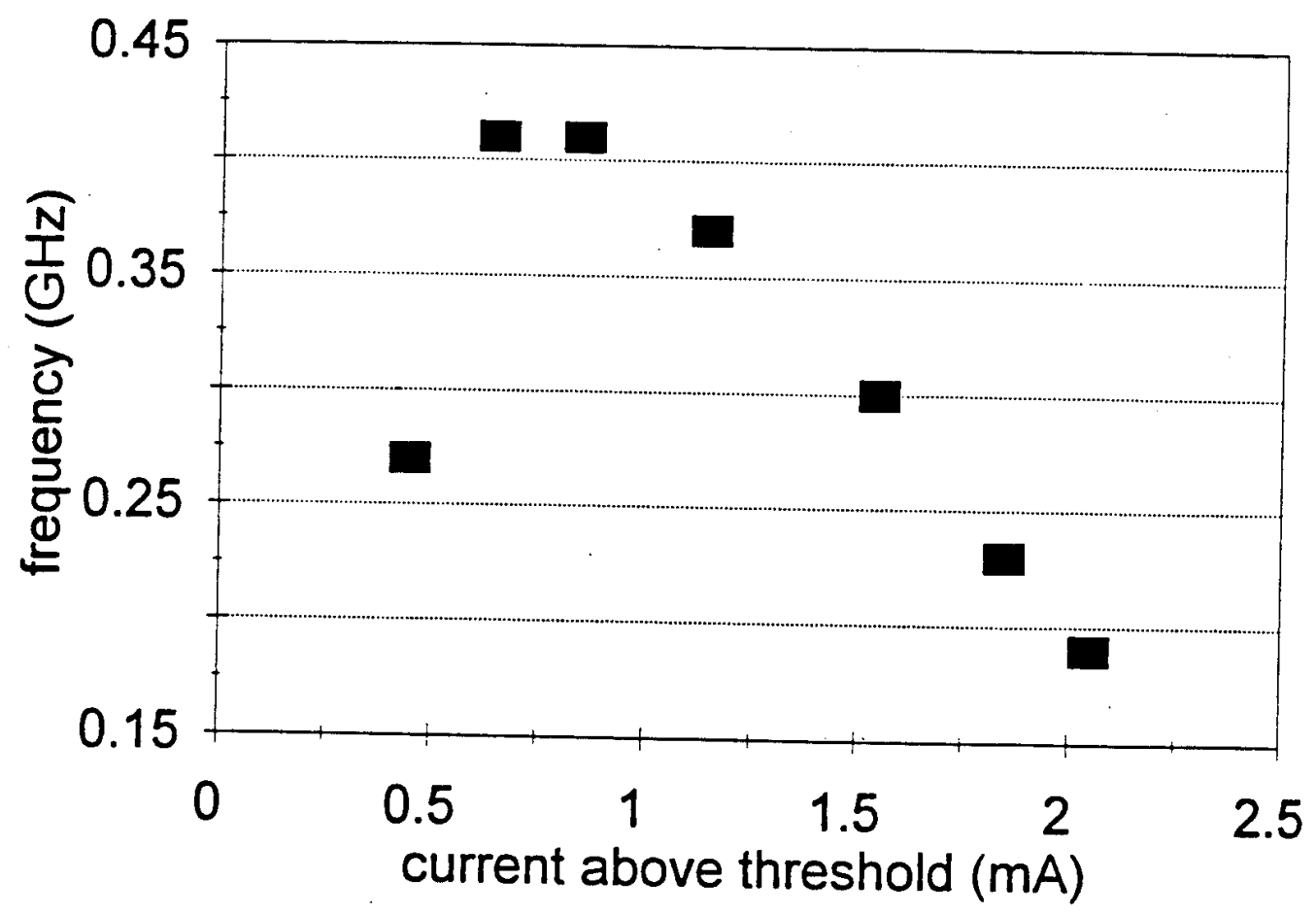

Figure 32. The frequency of the resonance due to polarization switching as a function of the injection current.

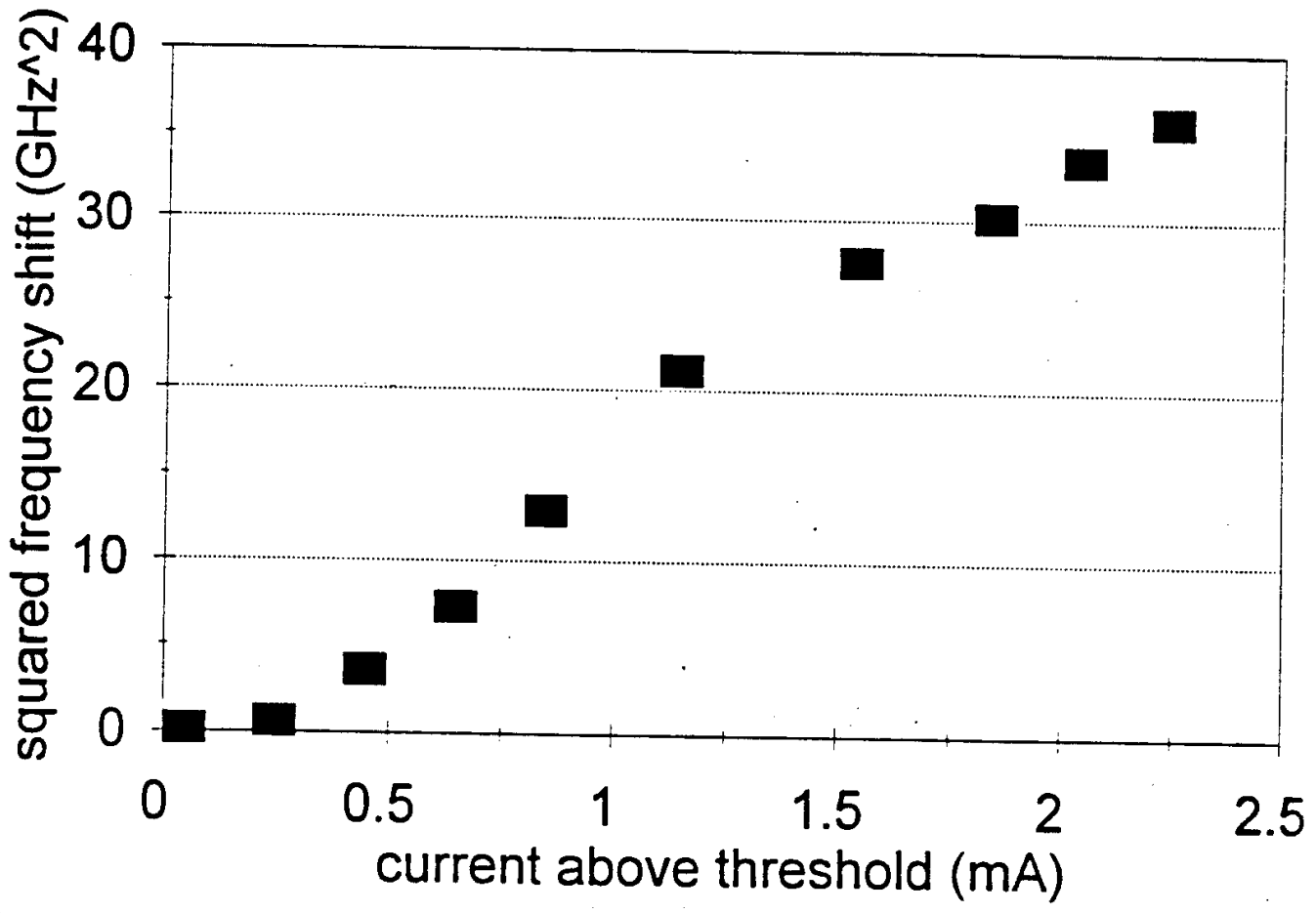

Figure 33. The dependence of the resonance peaks observed in the microwave spectra, regardless of polarization mode of operation, as a function of the injection current. The output power is approximately proportional to the operating current minus the threshold current. The resonance frequency exhibits the square root dependence on output power associated with the relaxation resonance. 
The VCSELs have been the subject of many papers in the literature. Dynamic characteristics of a free-running InGaAs quantum-well VCSEL [Ref. 55] are similar to the results presented here and have been modeled using standard single-mode laser diode models similar to the one described in this report. Instabilities in VCSELs under external optical injection [Ref. 56] and due to external optical feedback [Ref. 54] have been observed and also modeled using conventional laser diode models. However, this past modeling has always estimated basic laser parameters without determining the key rate parameters in the self-consistent fashion that we describe in this work. Multitransverse mode characteristics [Ref. 53] and polarization characteristics [Ref. 51,57] described in the literature mention characteristics similar to those we observe. Polarization characteristics are correlated with the overlap of the optical resonance of the cavity and the laser gain of the different polarization states [Ref. 57]. More work is necessary, especially in the region of nonlinear dynamics, to fully characterize these structures. 


\subsection{CONCLUSIONS}

The single-mode coupled-equation dynamical model of a semiconductor laser is an excellent tool for describing the operating characteristics of a nearly single-mode, quantum-well laser diode. Excellent quantitative agreement between the model and experimental data is observed. Both the noise spectra of the free-running laser and the spectra under external optical injection are welldescribed by the model. The effects of weak side modes on the noise spectrum can be included by the simple addition of an appropriate noise source to the carrier equation. We have shown how our combined modeling and experimental technique allows us to experimentally determine all of the parameters necessary for full, nonlinear numerical calculations with the model.

The nonlinear dynamics of a laser diode under external optic injection is sensitive to a variety of laser parameters. To date, we have demonstrated the existence of a region of chaotic dynamics bounded by period-doubling cascades, and a second region of period doubling, for optical injection at the free-running frequency of the laser diode. Detuning the injection frequency changes the nonlinear dynamics, as we have observed, and is predicted to lead to different routes to chaos [Reffs. 12, 34, 50]. In our analysis, the complete influence of the side modes was not included. The single-mode model used here cannot account for the partition of power to side modes; further work is needed to quantify the effect of the side modes.

Many areas for future work are opened by this research work. Two areas deserve special consideration. The first area is the modification of the modulation and bandwidth characteristics through the nonlinear optical interaction. Understanding this physics is crucial if we are to be able to make predictions about how optical injection can improve the dynamic performance characteristics of laser diodes. Laser diodes are expected to find many applications in optical communications and signal processing systems where their bandwidth and noise properties must be improved relative to currently available structures. In addition, their sensitivity to the effects of spurious optical feedback must be controlled. Our experimental results indicate that the bandwidth and noise characteristics can be significantly improved through the nonlinear optical coupling of an external signal. Recent theoretical work also indicates that chaotic dynamics can be brought under control by the introduction of a control parameter such as external optical injection [Refs. 58, 59].

The second key area is the extension of the current model to multimode structures such as coupled arrays and broad area emitters. To date, our results have been confined to laser diodes where the side modes can be modeled as a weak perturbation. Because of their small size and 
good efficiency, laser diodes which can deliver high power are of great interest. Such structures will, most likely, have the ability to support many propagating modes which will be mutually coupled. In the coupled-mode formalism, each mode acts as an external source to all of the others. If the coupling between modes is too weak, spontaneous emission noise will prevent the coherent locking of the participating modes and greatly reduce the maximum achievable brightness. If the coupling between modes is too strong, the modes are locked in an unstable or even chaotic manner and, again, ideal output cannot be achieved [Refs. 60,61]. However, with an external optical signal also coupled into the modes, it may be possible to sufficiently influence the multimode dynamics to produce a useful, coherent output.

Understanding the underlying physics of the nonlinear optics and dynamics in semiconductor lasers in these two key areas requires extensions in both our experimental and analytical/ modeling work. In the experimental program, we will have to investigate new types of laser diode structures. Further work on VCSELs is warranted. Spontaneous emission noise is a much stronger influence in these structures. Along with further studies of more conventional edgeemitting structures, studies of VCSELs will help determine how optical injection can modify the noise and modulation/bandwidth characteristics of laser diodes. A second semiconductor laser which can be usefully studied is the broad area edge emitter or similarly, the closely coupled linear array. These are the two structures that have been emphasized to increase the output power of individual laser diodes to powers well in excess of $1 \mathrm{~W}$. Both types are examples of multiple transverse-mode laser diodes with strongly coupled modes. In addition, unlike the case of a multimode VCSEL, or multiple longitudinal mode, single transverse-mode edge emitter, these lasers have modes which oscillate at closely spaced natural frequencies. This has an impact on the modeling because the carrier dynamics become sensitive to the intermode beat frequencies.

The coordinated experimental and modeling efforts have been very successful in the current program. This work has established the coupled-equation formalism as appropriate for quantitative, as well as qualitative, modeling of semiconductor laser devices. It has also shown the link between spontaneous-emission noise and nonlinear optical coupling. Understanding the noise and , uur-wave mixing interactions has allowed us to model the full nonlinear dynamics of a laser diode under external optical injection. This work provides a firm foundation for further studies of nonlinear dynamics and nonlinear optical coupling in semiconductor lasers. 


\section{REFERENCES}

1. Nakajima, H. and Frey, R., "Observation of Bistable Reflectivity of a Phase-Conjugated Signal Through Intracavity Nearly Degenerate Four-Wave Mixing," Physical Review Letters, Vol. 54, No. 16, pp. 1798-1801, April 1985.

2. Nakajima, H. and Frey, R., "Intracavity Nearly Degenerate Four-Wave Mixing in a (GaAl)As Semiconductor Laser," Applied Physics Letters, Vol. 47, No. 8, pp. 769-771, October 1985.

3. Frey, R., "On-Axis Intracavity Nearly Degenerate Four-Wave Mixing in Lasers," Optics Letters, Vol. 11, No. 2, pp. 91-93, February 1986.

4. Agrawal, G.P., "Four-Wave Mixing and Phase Conjugation in Semiconductor Laser Media," Optics Letters, Vol. 12, No. 4, pp. 260-262, April 1987.

5. Agrawal, G.P., "Population Pulsations and Nondegenerate Four-Wave Mixing in Semiconductor Lasers and Amplifiers," Journal of Optical Society of America B, Vol. 5, No. 1, pp. 147-158, January 1988.

6. Gallion, P., Debarge, G., and Chabran, C., "Output Spectrum of an Unlocked Optically Driven Semiconductor Laser," Optics Letters, Vol. 11, No. 5, pp. 294-296, May 1986.

7. Simpson, T.B. and Liu, J.M., Waveguide Four-Wave Mixing,Technical Report PL-TR-911045, Phillips Laboratory, Kirtland AFB, New Mexico, Technical Report, October 1991.

8. Vahala, K., Harder, Ch., and Yariv, A., "Observation of Relaxation Resonance Effects in the Field Spectrum of Semiconductor Lasers," Applied Physics Letters, Vol. 42, No. 3, pp. 211-213, 1 February 1983.

9. Henry, C.H., "Phase Noise in Semiconductor Lasers," IEEE Journal of Lightwave Technology, Vol. LT-4, No. 3, pp. 298-311, March 1986.

10. Agrawal, G.P. and Dutta, N.K., Long-Wavelength Semiconductor Lasers, Van Nostrand Reinhold, New York, New York, 1986.

11. Vahala, K. and Yariv, A.,"Semiclassical Theory of Noise in Semiconductor Lasers-Part II," IEEE Journal of Quantum Electronics, Vol. QE-19, No. 6, pp. 1102-1109, June 1983.

12. Tredicce, J.R., Arecchi, F.T., Lippi, G.L., and Puccioni, G.P., "Instabilities in Lasers with an Injected Signal," Journal of Optical Society of America B, Vol. 2, No. 1, pp. 173-183, January 1985 .

13. van Exter, M.P., Hamel, W.A., Woerdman, J.P., and Zeijlmans, B.R.P., "Spectral Signature of Relaxation Oscillations in Semiconductor Lasers," IEEE Journal of Quantum Electronics, Vol. 28, No. 6, pp. 1470-1478, June 1992.

14. Vahala, K.J. and Newkirk, M.A., "Parasitic-Free Modulation of Semiconductor Lasers," IEEE Journal of Quantum Electronics, Vol. 25, No. 6, pp. 1393-1398, June 1989.

15. Olshansky, R., Hill, P., Lanzisera, V., and Powazinik, W., "Frequency Response of $1.3 \mu \mathrm{m}$ InGaAs High Speed Semiconductor Lasers,". IEEE Journal of Quantum Electronics, Vol. QE-23, No. 9, pp. 1410-1418, September 1987. 
16. Su, C.B., Eom, J., Lange, C.H., Kim, C.B., Lauer, R.B., Rideout, W.C., and LaCourse, J.S., "Characterization of the Dynamics of Semiconductor Lasers Using Optical Modulation," IEEE Journal of Quantum Electronics, Vol. 28, No. 1, pp. 118-127, January 1992.

17. Inoue, K., Mukai, T., and Saitoh, T., "Nearly Degenerate Four-Wave Mixing in a Traveling-Wave Semiconductor Laser Amplifier," Applied Physics Letters, Vol. 51, No. 14, pp. 1051-1053, October 1987.

18. Stover, H.L.,"Theoretical Explanation for the Output Spectra of Unlocked Driven Oscillators," Proceedings of the IEEE, Vol. 54, No. 2, pp. 310-311, February 1966.

19. Simpson, T.B. and Liu, J.M.,"Phase and Amplitude Characteristics of Nearly Degenerate Four-Wave Mixing in Fabry-Perot Semiconductor Lasers," Journal of Applied Physics, Vol. 73, No. 5, pp. 2587-2589, 1 March 1993.

20. Hui, R. and Mecozzi, A., "Phase Noise of Four-Wave Mixing in Semiconductor Lasers," Applied Physics Letters, Vol. 60, No. 20, pp. 2454-2456, 18 May 1992.

21. Liu, J.M. and Simpson, T.B., "Characterization of Fundamental Parameters of a Semiconductor Laser with an Injected Optical Probe," IEEE Photonics Technology Letters, Vol. 4, No. 4, pp. 380-382, April 1993.

22. Jäckel, H. and Guekos, G., "High Frequency Intensity Noise Spectra of Axial Mode Groups in the Radiation from cw GaAlAs Diode Lasers," Optical and Quantum Electronics. Vol. 9, No. 3, pp. 233-239, May 1977.

23. Lopes, W.A., Morales, M.O., Rieffel, M.A., Giacoletti, J.A., Thorpe, J.T., and Westling, L.A., "Intensity Autocorrelation Measurements of an AlGaAs Diode Laser," Optics Letters,
Vol. 18, No. 10, pp. 820-822, 15 May 1993 .

24. Henry, C.H., Henry, P.S., and Lax, M., "Partition Fluctuations in Nearly SingleLongitudinal-Mode Lasers," IEEE Journal of Lightwave Technology, Vol. LT-2, No. 3, pp.
209-216, June 1984 .

25. Agrawal, G.P., "Mode-Partition Noise and Intensity Correlation in a Two-Mode Semiconductor Laser," Physical Review A, Vol. 37, No. 7, pp. 2488-2494, 1 April 1988.

26. Gray, G. and Roy, R., "Noise in Nearly-Single-Mode Semiconductor Lasers," Physical Review A, Vol. 40, No. 5, pp. 2452-2462, I September 1989.

27. Inoue, S., Ohzu, H., Machida, S., and Yamamoto, Y., "Quantum Correlation Between Longitudinal-Mode Intensities in a Multimode Squeezed Semiconductor Laser," Physical Review A, Vol. 46, No. 5, pp. 2757-2765, 1 September 1992.

28. Freeman, M.J. , Wang, H., Steel, D.G., Craig, R., and Scifres, D.R., "Amplitude-Squeezed Light from Quantum-Well Lasers," Optics Letters, Vol. 18, No. 5, pp. 379-381, 1 March
1993.

29. Freeman, M.J., Wang, H., Steel, D.G., Craig, R., and Scifres, D.R.,"Wavelength-Tunable Amplitude-Squeezed Light from a Room-Temperature Quantum-Well Laser," Optics Letters, Vol. 18, No. 24, pp. 2141-2143, 15 December 1993. 
30. Yamamoto, Y. and Imoto, N., "Internal and External Field Fluctuations of a Laser Oscillator: Part I-Quantum Mechanical Langevin Treatment," IEEE Journal of Ouantum Electronics, Vol. QE-22, No. 10, pp. 2032-2042, October 1986.

31. Yamamoto, Y., Machida, S., and Nilsson, O., "Amplitude Squeezing in a Pump-NoiseSuppressed Laser Oscillator," Physical Review A, Vol. 34, No. 5, pp. 4025-4042, November 1986.

32. "Nonlinear Dynamics in Optical Systems Technical Digest," Optical Society of America Vol. 16, No. 2, Washington, District of Columbia, 1992.

33. Roy, R., editor, "Chaos in Optics", SPIE. Proc. SPIE 2039, Bellingham, Washington, 1993.

34. Bandy, D.K., Narducci, L.M., and Lugiato, L.A., "Coexisting Attractors in a Laser with an Injected Signal," Journal of Optical Society of America B, Vol. 2, No. 1, pp. 148-155,

35. Otsuka, $\mathrm{K}$. and Kawaguchi, H., "Period-Doubling Bifurcations in Detuned Lasers with Injected Signals," Physical Review A, Vol. 29, No. 5, pp. 2953-2956, May 1984.

36. Boulnois, J.L., van Lerberghe, A., Cottin, P., Arecchi, F.T., and Puccioni, G.P., "Self Pulsing in a $\mathrm{CO}_{2}$ Ring Laser with an Injected Signal," Optics Communications, Vol. 58,
No. 2, pp. 124-129, 15 May 1986.

37. Sacher, J., Baums, D., Panknin, P., Elsässer, W., and Göbel, E.O., "Intensity Instabilities of Semiconductor Lasers Under Current Modulation, External Light Injection, and Delayed Feedback," Physical Review A, Vol. 45, No. 3, pp. 1893-1905, 1 February 1992.

38. Simpson, T.B. and Liu, J.M., "Spontaneous Emission, Four-Wave-Mixing and Noise in Laser Diodes," Optics Communications, Vol. 112, No. 1, pp. 43-47, 1 November 1994.

39. Mork, J., Mark, J. and Tromborg, B., "Route to Chaos and Competition Between Relaxation Oscillations for a Semiconductor Laser with Optical Feedback," Physical Review Letters, Vol. 65, No. 16, pp. 1999-2002, 15 October 1990.

40. Simpson, T.B., Liu, J.M., Gavrielides, A., Kovanis, V., and Alsing, P.M., "Period-Doubling Route to Chaos in a Semiconductor Laser Subject to External Optical Injection," Applied Physics Letters, Vol. 64, No. 26, pp. 3539-3541, 27 June 1994.

41. Petitbon, I., Gallion, P., Debarge, G., and Chabran, C., "Locking Bandwidth and Relaxation Oscillations of an Injection-Locked Semiconductor Laser," IEEE Journal of Quantum Electronics, Vol. 24, No. 2, pp. 148-154, February 1988.

42. Parker, T.S. and Chua, L.O., Practical Numerical Algorithms for Chaotic Systems. Springer-Verlag, New York, New York, 1989.

43. Bier, M. and Bountis, T.C., "Remerging Feigenbaum Trees in Dynamical Systems," Physics Letters, Vol. 104A, No. 5, pp. 239-244, 3 September 1984.

44. Oppo, G.L. and Politi, A., "Collision of Feigenbaum Cascades," Physical Review A.
Vol. 30, No. 1, pp. 435-441,.July 1984. 
45. Cascais, J., Dilão, R. and Noronha da Casta, A., "Chaos and Reverse Bifurcation in a RCL Circuit," Physics Letters, Vol. 93A, No. 5, pp. 213-216, 17 January 1983.

46. Bocko, M.F., Douglass, D.H., and Frutchy, H.H., "Bounded Regions of Chaotic Behavior in the Control Parameter Space of a Driven Non-linear Resonator," Physics Letters A,
Vol. 104, No. 8, pp. 388-390, 17 September 1984.

47. Tanaka, S. Matsumoto, T., Noguchi, J., and Chua, L.O., "Multi-Folding: Alternative Appearance of Period-One Attractors and Chaotic Attractors in a Driven R-L-Diode Circuit," Physics Letters A, Vol. 157, No. 1, pp. 37-43, 15 July 1991.

48. Lepers, C., Legrand, J., and Glorieux, P., "Experimental Investigation of the Collision of 1 March 1991 .

49. Annovazzi-Lodi, V., Donati, S., and Manna, M., "Chaos and Locking in a Semiconductor Laser Due to External Injection," IEEE Journal of Quantum Electronics, Vol. 30, No. 7,
pp. 1537-1540, July 1994.

50. Lee, E.K., Pang, H.-S., Park, J.D., and Lee, H., "Bistability and Chaos in an InjectionLocked Semiconductor Laser," Physical Review A, Vol. 47, No. 1, pp. 736-739, January

51. Choquette, Jr., K.D., Schneider, R.P., and Hunt, J.A., "Lasing Characteristics of Visible AlGaInP/AlGaAs Vertical-Cavity Lasers," Optics Letters, Vol. 19, No. 13, pp. 969-971,
1 July 1994. 52. Yokoyama, H., "Physics and Device Applications of Optical Microcavities," Science,
Vol. 256, No. 14, pp. 66-70, 3 April 1992.

53. Tai, K., Lai, Y., Huang, K.F., Huang, T.C., Lee, T.D., and Wu, C.C., "Transverse Mode Rmission Characteristics of Gain-Guided Surface Emitting Lasers," Applied Physics
Letters, Vol. 63, No. 19, pp. 2624-2646, 8 November 1994.

54. Chen, H.M., Tai, K., Huang, K.F., Kao, Y.H., and Wynn, J.D., "Instability in Surface Emitting Lasers Due to External Feedback," Journal of Applied Physics, Vol. 73, No. 1,
pp. 16-19, 1 January 1993.

55. Tauber, D., Wang, G., Geels, R.S., Bowers, J.E., and Coldren, L.A., "Large and Small Signal Dynamics of Vertical Cavity Surface Emitting Lasers," Applied Physics Letters, Vol. 62, No. 4, pp. 325-327, 25 January 1993.

56. Boggavarapu, D., Jin, R., Grantham, J., Hu, Y.Z., Brown de Colstoun, F., Lowry, C.W., Khitrova,G., Koch, S.W., Sargent III, M., and Gibbs, H.M., "Instabilities of a Microcavity Laser With a Weak Injected Signal," Optics Letters, Vol. 18, No. 21, pp. 1846-1848,
1 November 1993.

57. Choquette, K.D., Richie, D.A., and Leibenguth, R.E., "Temperature Dependence of GainGuided Vertical-Cavity Surface Emitting Laser Polarization," Applied Physics Letters. Vol. 64, No. 16, pp. 2062-2064, 18 April 1994.

58. Newell, T.C., Alsing, P.M., Gavrielides, A., and Kovanis, V., "Synchronization of Chaotic Diode Resonators by Occasional Proportional Feedback," Physical Review Letters, Vol. 72,
No. 11, pp. 1647-1650, 14 March 1994. 
59. Newell, T.C., Alsing, P.M., Gavrielides, A., and Kovanis, V., "Synchronization of Chaos Using Proportional Feedback," Physical Review E, Vol. 49, No. 1, pp. 313-320, January 1994.

60. Wang, S.S. and Winful, H.G., "Dynamics of Phase-Locked Semiconductor Laser Arrays," Applied Physics Letters, Vol. 52, No. 21, pp. 1774-1776, 23 May 1988.

61. Winful, H.G. and Wang, S.S., "Stability of Phase Locking in Coupled Semiconductor Laser Arrays," Applied Physics Letters, Vol. 53, No. 20, pp. 1894-1896, 15 November 1988. 
PL-TR--94-1161

\section{DISTRIBUTION}

AFSAA/SAI, Washington DC

AUL/LSE, Maxwell AFB, AL

DTIC/OCC, Alexandria, VA

$\mathrm{PL} / \mathrm{SUL} / \mathrm{HO}$, Kirtland AFB, NM

Dr T. B. Simpson, Jaycor, San Diego, CA

Official Record Copy, (PL/LIDN, Dr Chris Clayton) 